Nuclear Physics B271 (1986) 93-165

North-Holland, Amsterdam

\title{
CONFORMAL INVARIANCE, SUPERSYMMETRY \\ AND STRING THEORY
}

\author{
Daniel FRIEDAN ${ }^{1}$ \\ Enrico Fermi and James Franck Institutes and Department of Physics, University of Chicago, \\ Chicago, IL 60637, USA \\ Emil MARTINEC ${ }^{2}$ \\ Joseph Henry Laboratories, Princeton University, Princeton, NJ 08544, USA \\ Stephen SHENKER ${ }^{1}$ \\ Enrico Fermi and James Franck Institutes and Department of Physics, University of Chicago, \\ Chicago, IL 606.37, USA
}

Received 16 December 1985

\begin{abstract}
Covariant quantization of string theories is developed in the context of conformal field theory and the BRST quantization procedure. The BRST method is used to covariantly quantize superstrings, and in particular to construct the vertex operators for string emission as well as the supersymmetry charge. The calculation of string loop diagrams is sketched. We discuss how conformal methods can be used to study string compactification and dynamics.
\end{abstract}

\section{Introduction}

The past year has seen the opening of a new era in the search for a fundamental physical theory. In the past, attempts at unification of the forces of nature have focussed on enlarging the symmetry group of short distance physics - large nonabelian gauge groups [1], supersymmetry [2] higher dimensions [3] etc. Dual string theories [4] are a radical step in this direction, incorporating the infinite-dimensional algebra of two-dimensional reparametrizations (and perhaps affine Lie algebras as well [5]) into the picture. This vast increase in symmetry is accompanied by correspondingly severe restrictions on the structure of the theory. Nature seems to enjoy running on the verge of inconsistency; the more potential anomalies a theory has, without actually being anomalous, the closer it seems to be to physical reality.

\footnotetext{
${ }^{1}$ Supported in part by DOE grant DE-FG02-84ER-45144 and the Alfred P. Sloan Foundation

${ }^{2}$ Supported in part by DOE grant DE-AC02-76ER-03072.
} 
Actually, local and global gauge and reparametrization anomalies are helpful in constraining the content of theories. The two-dimensional reparametrization group of string theories provides it with a rich structure of anomalies. One particularly important subgroup is that of conformal reparametrizations, the conformal group [6]. One may fix a covariant gauge condition upon quantization which leaves conformal transformations as a residual symmetry; this large remnant of coordinate invariance has proved an extremely useful tool in the analysis of string theories. In the days of hadronic dual models, conformal invariance was used as a consistency requirement [7]. It is now understood that avoidance of local and global conformal anomalies is responsible for the critical dimension $d=10$ (or 26) [8,9], spacetime supersymmetry [10], and restrictions on possible gauge groups $[11,12]$. The alternative light-cone gauge quantization has proven to be extremely useful in initially formulating the theory [9] and carrying out basic calculations, but it is our belief that covariant methods will yield greater insight, especially in unravelling whatever nonlinear invariance underlies string theory.

In its current state, string theory lacks an organizing principle. Historically, much of dual theory was worked out before a string interpretation was developed. The present state of the art consists of a prescription for computing string $S$-matrix elements about some fixed background; the deep connection between the string and the environment in which the string lives remains to be uncovered. Some idea unifies background and quantum fluctuations, combining the algebraic structure of the string vertex algebra with spacetime general coordinate and local gauge symmetry; conformal invariance seems to be the key component of that idea.

In this work we shall undertake an exploration of the conformal structure of string theory using techniques in two-dimensional conformal field theory [13,14]. A rather brief description of this structure was set forth in a previous letter [15]; here we present the details of the formalism. Elements of the construction were worked out independently in [15a]. A great deal of the material covered here is scattered throughout the early literature on dual resonance models. We have endeavored to reference the work we are aware of, but our knowledge of history is incomplete. Those wishing to delve further into the literature should consult previous reviews [4]. The language of conformal field theory provides a concise statement of these previously known results as well as an indispensible tool in our further development of the covariant approach.

showing how the structure of the string theory is built on its conformal properties. We review the general properties of two-dimensional conformally invariant field theories, indicating how the conformal algebra together with the operator product algebra of conformal fields determines all the correlation functions. We also quantize the Faddeev-Popov ghosts of the conformal gauge $[8,16,17]$ and connect the conformal algebra with the BRST quantization method [18]. Our description of strings is first-quantized since we quantize the motion of single strings rather than fields of string. Sums over surfaces represent the Feynman rules for string theory. 
The ghosts provide the appropriate compensating factors in loop graphs for the covariant gauge. They also clarify some aspects of the old operator formalism, such as the negative shift in the ground state energy which generates the tachyon.

Sect. 3 is an exposition of superconformal field theory, i.e. $2 \mathrm{~d}$ supersymmetric conformal field theory. Just as the algebra of conformal transformations is infinitedimensional, conformal supersymmetry is likewise an infinite-dimensional algebra. In addition, it comes in two varieties [19-22,62] because fermions may be doublevalued on the string world sheet. The additional operators (called spin operators) which interchange periodic and antiperiodic $2 \mathrm{~d}$ fermion boundary conditions [22, 22a] are an integral part of the construction of the fermionic string theory [14,15]. We use them in sects. 4 and 5 to build the spacetime fermion vertex and the spacetime supersymmetry charge of the fermionic string. The Faddeev-Popov ghosts for local supersymmetry also enter into this construction in an essential way; without them, one could not make fermion vertices with the requisite conformal properties. In sect. 4 we also discuss the world-sheet superfields of the fermionic string, and how they intertwine with the spin operators. The $2 \mathrm{~d}$ superpartners of the string coordinates are shown to be completely described as a representation of a Lorentz group affine algebra. A constant theme of our work is the use of current algebra structures to simplify the analysis of strings. Here the Lorentz current algebra facilitates the treatment of operators with spacetime fermion quantum numbers.

Sect. 5 finishes the covariant quantization of fermionic strings with an in-depth analysis of the BRST procedure and the superconformal ghost field theory. The fermion vertex operators have a rather elaborate structure having to do with the fact that they contain the ghosts; nevertheless, the theory is unitary. The algebra of the ghost current plays an important role; it enables us to find the operators needed for a complete description of the fermion sector. We also present some sample calculations of string scattering amplitudes and explain how conformal techniques are used to calculate correlation functions. In sect. 6 we discuss some aspects of supersymmetry in the covariant formalism, showing how various correlation functions are related by supersymmetry. We also conjecture a relationship between our covariant quantization procedure and other potential covariant quantizations $[23,24]$. Sect. 7 contains an introduction to the calculation of string loop graphs, adopting methods developed in the heyday of the dual resonance model.

As mentioned above, string theory must self-consistently determine the geometry of spacetime. A first step in understanding how this comes about is provided by the study of string propagation in general background fields [25-27]. The conformal algebra structure generalizes to any scale invariant $2 \mathrm{~d}$ quantum field theory, and could provide a unified picture of string dynamics. Therefore, in sect. 8 we discuss the generalization of the fermionic string away from flat space. Another motivation for this exercise is to find candidate vacuum solutions for the purposes of phenomenology [26]. Given a conformal field theory which describes a solution to the string equations, we indicate how the conformal and operator product algebras encode 
information about the low-energy effective theory of the string. In sect. 9 we present our conclusions.

\section{Conformal field theory and the bosonic string}

\subsection{INTRODUCTION}

We begin our discussion with a rather detailed account of the bosonic string. The language we develop will have direct application when we consider the fermionic string later on. The world sheet of a propagating bosonic string is described by a map $X(\xi)$ from a two-dimensional parameter space manifold $S, \xi \in S$, into a spacetime $\mathscr{T}$. The path integral over such surfaces yields a first-quantized description since the basic object is a string trajectory rather than a function(al) on strings. The geometry of $\mathfrak{O}$ is specified a priori, and the string represents a fluctuation propagating on this background. Because the string theory contains quantum gravity, the dynamics in principle determines $\mathscr{T}$. A proper treatment of this problem must await a second quantized formalism ${ }^{\star}$ where general coordinate invariance, or its string generalization, is manifest. Even so, one can examine whether strings propagate consistently (without ghosts, anomalies, or tadpoles) on OR. The requirement is equivalent to demanding conformal invariance of the first-quantized string theory and may be used to search for the allowed ground states of the string [27] and for the classical equations of motion of the string acting as background geometry. We will return to this point in due course, but for the moment, let us consider $\Re$ to be flat, $d$-dimensional Minkowski spacetime. The map $X$ is then given by $d$ fields $X^{\mu}(\xi), \mu=0, \ldots, d-1$. The action governing string dynamics should be geometrical and therefore cannot depend on the choice of coordinates for either $\subseteq$ or $\mathfrak{T}$. The most general Poincaré invariant, reparametrization invariant, renormalizable action in flat space is $[8,29]$

$$
S=\int \mathrm{d}^{2} \xi \sqrt{g}\left[g^{a b} \partial_{a} X^{\mu} \partial_{b} X^{\nu} G_{\mu \nu}(X)+R^{(2)}+\lambda\right]
$$

We have coupled the string fields $X^{\mu}$ to a two-dimensional metric $g_{a b}$ in order to ensure two-dimensional coordinate invariance; $R^{(2)}$ is the intrinsic curvature of this metric; and for simplicity we choose Cartesian coordinates in $\Re$ so that $G_{\mu \nu}=\eta_{\mu \nu}$. In calculating the path integral over surfaces

$$
Z=\int \mathscr{Q} g_{a b} \mathscr{D} X^{\mu} \mathrm{e}^{i S(g, X)}
$$

\footnotetext{
* Conformal field theory techniques may be of considerable help here, however, in sorting out the dynamics and the kinematics of string gravity [28].
} 
we must choose a gauge in order to remove the degeneracy of paths generated by coordinate transformations. Typically, one of two choices is made; one can either

(i) integrate over the Lagrange multipliers $\sqrt{g} g^{a b}$, fixing a gauge involving the $X^{\mu}$ $[9,30]$, or

(ii) fix the Lagrange multipliers by setting $[29,8]$

$$
g_{a b}=\mathrm{e}^{\sigma} \hat{g}_{a b},
$$

where $\hat{g}_{a b}$ is a fixed reference metric which will depend on several parameters for surfaces $\delta$ with handles. For instance, we can choose $\hat{g}_{a b}$ to be a constant curvature metric for the space $S$ adapted to light-like coordinates $\xi^{ \pm}$.

For case (i), the path integral now contains the $\delta$-function constraints $\left(\partial_{ \pm}=\partial_{0} \pm \partial_{1}\right)$

$$
\delta\left(\partial_{+} X \cdot \partial_{+} X\right) \delta\left(\partial_{-} X \cdot \partial_{-} X\right),
$$

which are quadratic in the fields. These constraints are easily eliminated if they can be linearized, which is achieved with the gauge choice [9]

$$
X^{+}=x^{+}+p^{+} \xi^{0}, \quad X^{ \pm}=X^{0} \pm X^{d-1} .
$$

Integrating now over $X^{ \pm}$yields the light-cone gauge-formalism. It has the advantage that all the gauge freedom has been eliminated, with the exception of constant shifts of the $\xi^{a}$ generated by the total hamiltonian and momentum

$$
\begin{aligned}
& H=\int \mathrm{d}^{2} \xi \frac{1}{2}\left[\left(\partial_{0} X\right)^{2}+\left(\partial_{1} X\right)^{2}\right], \\
& P=\int \mathrm{d}^{2} \xi\left[\partial_{0} X \cdot \partial_{1} X\right]
\end{aligned}
$$

as well as certain discrete diffeomorphisms (known as modular transformations) which act on world surfaces with $g$ handles. These residual invariances must be imposed as constraints on the Hilbert space of physical states [4]; they provide, for instance, some of the constraints which determine the structure of the heterotic string [12]. One unfortunate difficulty of the light-cone gauge is that $p^{+}$is not globally defined on surfaces of genus $g \geqslant 2$, which makes string perturbation theory calculations somewhat awkward. Also manifest Lorentz invariance is lost.

In the present discussion, we wish to consider instead case (ii), which manifestly preserves Lorentz invariance. Because this covariant gauge quantization fixes the Lagrange multipliers of the gauge constraint, and does not eliminate the negative metric field $X^{0}$, we will be left with an indefinite metric theory. All is not lost, though, because the gauge (2) is preserved by analytic coordinate transformations

$$
\xi^{ \pm} \rightarrow f\left(\xi^{ \pm}\right),
$$


generating the group of two-dimensional pseudo-conformal transformations [6]. Henceforth we will continue to euclidean space, where these are (anti)analytic transformations on complex coordinates $z, \bar{z}$. This residual invariance group guarantees that the negative metric states decouple from physical amplitudes $[31,32]$ much as $A^{0}$ decouples in the Gupta-Bleuler formalism for QED. Conformal invariance is typically anomalous in quantum field theory, however, so we must be more careful. It has been known for some time that string theories are only consistent in 26, 10 or 2 spacetime dimensions depending on whether they have $N=0,1$ or 2 world-sheet supersymmetries. Polyakov $[8,33]$ showed that precisely in these dimensions, the conformal anomalies of the string fields cancel with anomalies in the path integral measure associated with the jacobian for the (super)conformal gauge choice (ii). More recently, conformal field theory techniques have been used to solve a number of interesting problems in two-dimensional statistical mechanics [13,14], current algebra [34,35], and field theory [36]. The power of these techniques for analyzing the structure of a theory and computing correlation functions may be applied to string theory as well. Therefore we digress for a while to explain what is meant by conformal field theory.

\subsection{CONFORMAL FIELD THEORY ${ }^{\star}$}

In general, a two-dimensional field theory whose stress-energy tensor is traceless

$$
T_{a}^{a}=T_{z \bar{z}}=0, \quad T_{z \bar{z}}=T_{00}+T_{11}
$$

is not only dilation invariant but also respects the symmetries (3) which preserve the metric $\hat{g}_{a b}$. The local conformal fields [36a] $\phi_{j}$ of the theory (sometimes called primary fields) may be classified according to their conformal weights $h_{j}, \bar{h}_{j}$ under the dilations $z \rightarrow \lambda z, \bar{z} \rightarrow \bar{\lambda} \bar{z}$. In conventional terms $h_{j}+\bar{h}_{j}$ is the scaling dimension and $h_{j}-\bar{h}_{j}$ the spin of $\phi_{j}$. Ordinary tensors $\phi_{z z \ldots} \bar{z} \bar{z}(\mathrm{~d} z)^{n}(\mathrm{~d} \bar{z})^{m}$ have $h_{\phi}=n$, $\bar{h}_{\phi}=m$; in particular, the metric may be split into its trace and traceless parts

$$
\mathrm{d} s^{2}=g_{z \bar{z}} \mathrm{~d} z \mathrm{~d} \bar{z}+g_{z z} \mathrm{~d} z^{2}+\text { c.c. }
$$

The gauge choice (2) means that locally (but not necessarily globally) we may choose $g_{z \bar{z}}=\mathrm{e}^{2 \sigma}, g_{z z}=g_{\bar{z} \bar{z}}=0$. In field theory the Noether currents generating spacetime transformations on the fields are the moments of the stress-energy tensor. The transformations $z \rightarrow w(z), \bar{z} \rightarrow \bar{w}(\bar{z})$, change the conformal fields according to

$$
\phi_{j}(z, \bar{z}) \rightarrow\left(\frac{\partial w}{\partial z}\right)^{h_{j}}\left(\frac{\partial \bar{w}}{\partial \bar{z}}\right)^{\bar{h}_{j}} \phi_{j}(w, \bar{w}) .
$$

This may be regarded as the definition of a conformal field. Note that, due to the conservation laws $\partial_{\bar{z}} T_{z \bar{z}}=\partial_{z} T_{\bar{z} \bar{z}}=0, T_{\bar{z} \bar{z}}\left(T_{\bar{z} \bar{z}}\right)$ is an (anti)analytic function of $z(\bar{z})$.

* The discussion here follows $[13,14]$ and references therein. 
Anticipating the application to strings, it is natural to consider the dilation operator to be the hamiltonian of our field theory; we can map the cylinder swept out by a propagating string onto the complex plane via $z=\mathrm{e}^{t+i \sigma}$. Time ordering is then radial ordering; $t=$ const hypersurfaces are circles concentric about the origin of the $z$-plane. More generally, the local operator product relations extend naturally to arbitrary Riemann surfaces with local conformal coordinates $z, \bar{z}$. We usually specialize to the complex plane to simplify the presentation and to make use of operator and Hilbert space notions. The infinitesimal transformation $z \rightarrow z+f(z)$ is generated by

$$
\begin{array}{ll}
T_{f}=\oint_{\mathrm{C}} f^{z} T_{z z}, & T_{z z}=T_{11}-T_{22}-2 i T_{12} \equiv T(z), \\
\bar{T}_{\bar{f}}=\oint_{\mathrm{C}} f^{\bar{z}} T_{\bar{z} \bar{z}}, & T_{\bar{z} \bar{z}}=T_{11}-T_{22}+2 i T_{12} \equiv \bar{T}(\bar{z}),
\end{array}
$$

where $\mathrm{C}$ is a contour surrounding the origin. In correlation functions, the transformation (4) can be rewritten in terms of a contour integral $[37,38]$

$$
\begin{aligned}
\delta_{f} \phi_{j}=\left[T_{f}, \phi_{j}(w)\right] & =\left[\oint_{C_{0 w}} \frac{\mathrm{d} z}{2 \pi i}-\oint_{C_{0}} \frac{\mathrm{d} z}{2 \pi i}\right] f(z)\left\langle T(z) \phi_{j}(w)\right\rangle \\
& =\oint_{C_{n}} \frac{\mathrm{d} z}{2 \pi i} f(z)\left\langle T(z) \phi_{j}(w)\right\rangle .
\end{aligned}
$$

Here the contour $\mathrm{C}_{0 w}$ surrounds 0 and $w$ while $\mathrm{C}_{0}$ contains 0 but not $w$; radial ordering produces the commutator (see fig. 1). The last equality is obtained when the contour $\mathrm{C}_{0 w}-\mathrm{C}_{0}$ is deformed to a curve $\mathrm{C}_{w}$ about $w$, asymptotically close. This deformation is allowed because $T(z)$ is analytic in correlation functions everywhere except at the locations of operators. Since this contour is infinitesimally close to $w$ the information about conformal transformations is contained entirely in the operator product relations

$$
T(z) \phi_{j}(w, \bar{w}) \approx \frac{1}{(z-w)^{2}} h_{j} \phi_{j}(w, \bar{w})+\frac{1}{z-w} \partial_{w} \phi_{j}+\text { finite as } z \rightarrow w .
$$
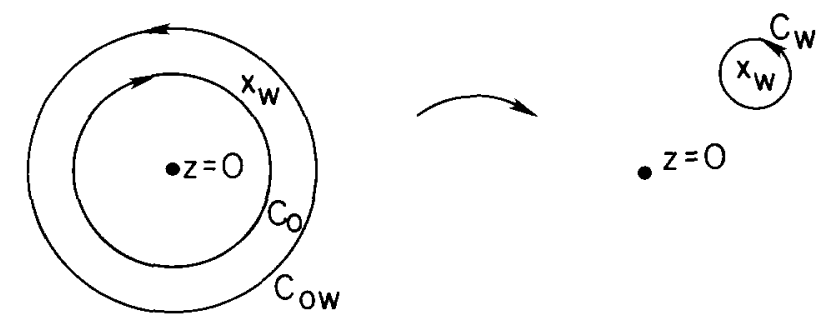

Fig. 1. The difference of two time-ordered contour integrations yields a commutator. 
We see that commutation relations are efficiently expressed in terms of contour integrals which pick up the singularities in operator product expansions. In most of what follows we shall use the operator product expansion rather than commutators; one can think of the operator product relations as generating functions for the commutators. The algebra of conformal transformations

$$
\begin{aligned}
{\left[L_{m}, L_{n}\right] } & =(m-n) L_{m+n}+\frac{1}{12} c\left(m^{3}-m\right) \delta_{m+n, 0}, \\
L_{n} & =\oint \frac{\mathrm{d} z}{2 \pi i} z^{n+1} T(z)
\end{aligned}
$$

is itself embodied in the operator product relations

$$
T(z) T(w) \approx \frac{c}{2(z-w)^{4}}+\frac{2}{(z-w)^{2}} T(w)+\frac{1}{z-w} \partial T(w) .
$$

The leading singularity yields the Schwinger term in (9); $c=1$ for a free field such as $X^{\mu}$. This anomaly describes the lack of invariance of the theory to deforming the background metric $\hat{g}_{a b}[38,39]$. The maximal closed subalgebra of the conformal group is $\operatorname{SL}(2, R)$, which is generated by $L_{0}, L_{ \pm 1}$; its commutation relations are unaffected by the anomaly and thus correlation functions on the complex plane will exhibit an invariance under

$$
z \rightarrow \gamma(z)=\frac{a z+b}{c z+d}, \quad a, b, c, d \in \mathbf{R}, \quad a d-b c=1 .
$$

For higher-genus world surfaces, this will not be true (see below). Of course, all the above considerations also apply to the $\bar{z}$-dependence of the theory, which may be treated in a similar fashion, so that in fact we have $\operatorname{SL}(2, \mathrm{C})$ (the parameters in (11) may be complex). We will focus on the $z$-dependence to avoid repetition.

On the sphere or the torus, correlation functions calculated from the path integral can also be written in an equivalent operator formulation in terms of operator expectations in a Hilbert space. For higher genus, there might be an interpretation in terms of some kind of "local Hilbert space" in the neighborhood of a point on the surface. The vacuum $|0\rangle$ of the $2 \mathrm{~d}$ field theory may be chosen to be an $\mathrm{SL}_{2}$ invariant state (i.e. invariant under translations, dilations, and special conformal transformations), with $L_{n}|0\rangle=0$ for $n \geqslant-1$. This follows from the vacuum expectation value of (10). The conformal fields $\phi_{j}$ acting on the vacuum create asymptotic "in" states $|j\rangle=\phi_{j}(0)|0\rangle$ with energy $L_{0}|j\rangle=h_{j}|j\rangle$ (recall that $z=0$ is $t=-\infty$ on the cylinder). There is a one-to-one correspondence between the fields and the states in Hilbert space that they create at $z=0$. The operator product (8) implies $L_{n}|j\rangle=0$, $n>0$. To see this, evaluate (8) at $w, \bar{w}=0$ and expand $T$ using (9). The $L_{n}, n>0$ in general lower the energy of a state by an amount $n$; conversely, the $L_{n}, n<0$ are 
raising operators. The states $|j\rangle$ are thus annihilated by all the lowering operators of the Virasoro operators and are called highest weight vectors because mathematicians reverse the sign of $L_{0}$.

From such a state we can build a representation of the Virasoro algebra by applying the $L_{-n}, n \geqslant 1$, much in the same way one builds representations of compact Lie groups using ladder operators. The states can be classified by $L_{0}$ eigenvalue ( $=$ energy); and $n$th level state has $L_{0}$ value $h_{j}+n$. A basis of $n$th level states in the family associated with $\phi_{j}$ is given by all the combinations of raising operators of total energy $n$ :

$$
L_{-k_{1}} L_{-k_{2}} \ldots L_{-k_{m}}|h\rangle, \quad \sum k_{i}=n, \quad k_{1} \geqslant k_{2} \geqslant \cdots \geqslant k_{m}>0 .
$$

These states have operator products with $T$ that are more singular than (8) because the positive frequency modes of $T$ don't commute with the $L_{-k_{i}}$ in (12). The tower of such levels is called a highest weight representation or Verma module $V\left(h_{j}\right)$ of the algebra. Since the full conformal group is a direct product of its analytic and antianalytic components, the representations are also direct products $V\left(h_{j}\right) \otimes \bar{V}\left(\bar{h}_{j}\right)$. When $c<1$ the structure provided by the conformal algebra in fact completely determines the theory $[13,14]$ - both the allowed representations $V\left(h_{j}\right)$ and the correlation functions.

In equivalent field theoretic language, these higher level states correspond to fields "descended" from the "ancestor" field $\phi_{j}$ by applying products of stress-energy tensors $T(z)$. One conclusion is that correlations of the descendants are determined from those of the ancestors via conformal Ward identities [13]. We shall often proceed in this manner, using operator and field theoretic methods interchangeably and employing whichever framework is most suited to the problem at hand.

Finally, the sets of fields $\phi_{j}$ and their descendants completely characterize the theory in that the product of any two fields may be represented as a sum [13]

$$
\left.\phi_{j}(z) \phi_{k}(0)=\sum_{n, \bar{m}} C_{j k}^{l\{n, \bar{m}\}} \phi\right\}^{n, \bar{m}\}}(0) z^{h_{i}+\Sigma\{n\}-h_{j}-h_{k} \bar{z}^{h_{l}}+\Sigma\{\bar{m}\}-\bar{h}_{j}-\bar{h}_{k}},
$$

where $\phi\}^{\{m\}}(0)$ are members of the family of operators generated from $\phi_{l}(0)$ by applying products of $L_{n}$ 's and $\bar{L}_{\bar{m}}$. Eq. (13) is essentially a Clebsch-Gordan series for the decomposition of two representations of the conformal algebra with highest weight vectors $\phi_{j}$ and $\phi_{k}$. Conformal invariance imposes powerful constraints on the form of the coefficients $C_{j k}^{f(n, \bar{m}\}}(z, \bar{z})$; for instance, the coefficients of the ancestor fields determines the daughter coefficients

$$
C_{j k}^{l\{n, \bar{m}\}}=C_{j k}^{l} \beta_{j k}^{l\{n\}} \beta_{j k}^{\prime\{\bar{m}\}},
$$

with the $\beta$ 's determined through the conformal algebra. Also, crossing symmetry of four-point functions relates quadratic sums of $C$ 's in the $s$ - and $t$-channels to give 
the so-called bootstrap constraints [13]; schematically, the operator product (13) implies

$$
\left.\left\langle\phi_{i} \phi_{j} \phi_{k} \phi_{l}\right\rangle=\sum_{p}{ }_{j}^{i}\right\rangle-p_{p}^{l}=\sum_{p j}^{i} \bigcup_{p}^{l},
$$

where the vertices are the operator product relations (13). These must be satisfied by any conformal field theory, and give a set of nonlinear relations among the $h_{i}$ and $C_{i j}{ }^{k}$.

It is a rather remarkable fact that a conformal field theory is completely specified by its spectrum of anomalous dimensions $h_{j}$ and operator product coefficients $C_{i j}{ }^{k}$. All correlation functions may in principle be calculated by repeated application of (13).

\subsection{STRING THEORY}

We now return to the string theory to elucidate its structure as a conformal field theory. In conformal gauge (2) the action (1) describes a set of $d$ free fields. The solution to the equations of motion $\partial_{z} \partial_{\bar{z}} X^{\mu}=0$ is $X^{\mu}(z, \bar{z})=x^{\mu}(z)+x^{\mu}(\bar{z})$. The two-point function (on the complex plane)

$$
\left\langle X^{\mu}(z, \bar{z}) X^{\mu}(w, \bar{w})\right\rangle=-g^{\mu \nu} \ln |z-w|^{2}
$$

demonstrates that the fields $X^{\mu}$ do not have a well-defined scaling dimension; they do not rigorously exist as quantum fields [40]. Nevertheless, we shall only need to consider conformal fields such as $\partial X^{\mu}$ of dimension 1 and $\mathrm{e}^{i p \cdot X}$ of dimension $\frac{1}{2} p^{2}$. Eq. (15) has finite corrections on higher genus surfaces to be discussed in sect. 7. The stress tensor

$$
T(z)=-\frac{1}{2} \partial_{z} X \cdot \partial_{z} X
$$

satisfies the algebra (9), (10) with $c=d$. By eq. (16) (and similar expressions below) we mean the normal ordered product of operators, which may be obtained by subtraction of the singular part of the operator product expansion

$$
: \partial_{z} X \partial_{z} X:=\left[\partial_{z} X \partial_{w} X-\frac{1}{(z-w)^{2}}\right]_{z \rightarrow w} .
$$

The Laurent expansion coefficients $L_{n}$ are the gauge operators which decouple negative metric states $[31,32,4]$; thus physical states satisfy

$$
\left.L_{n} \mid \text { phys }\right\rangle=0, \quad n>0 .
$$

This is precisely the condition that $|\mathrm{phys}\rangle=V_{\text {phys }}(0)|0\rangle$ be a highest weight vector of the Virasoro algebra. The string vertex operators creating such states are therefore conformal fields; the spurious states are created by their descendants. 
Because of the anomaly term in the commutation relations (9), one cannot demand that all the generators of conformal reparametrization annihilate physical states; the best that can be done is (17). However the remaining reparametrization generators $L_{n}, n<0$ generate spurious states which decouple from physical processes. In this sense string theory is invariant under conformal reparametrizations of the fields. As mentioned above, the mechanism is similar to the decoupling of longitudinal photons in the indefinite metric quantization of QED (but rather more subtle). As for the middle generator $L_{0}$, reparametrization invariance dictates that physical vertices have dimension one in both $z$ and $\bar{z}$ (denoted by $h=(1,1)$ ) so that their integral against $\mathrm{d} z \mathrm{~d} \bar{z}$ is invariant. The standard example is the first excited level, where the physical state conditions are those of massless gravitons, antisymmetric tensors, and dilatons. To see how the conditions (17) work in general, consider the second mass level. Candidate conformal fields are $V_{1}^{\mu \nu}=\partial X^{\mu} \partial X^{\nu} \mathrm{e}^{i k \cdot X}(z)$ and $V_{2}^{\mu}=\partial^{2} X^{\mu} \mathrm{e}^{i k \cdot X}(z), k^{2}=-2$; we focus on the $z$-dependence - the same considerations independently apply to the $\bar{z}$ dependence. The operator products of $V_{1}$ and $V_{2}$ with the stress tensor are

$$
\begin{aligned}
T(z) V_{1}^{\mu \nu}(w) \approx & \frac{1}{(z-w)^{4}} \eta_{\mu \nu} \mathrm{e}^{i k \cdot X}+\frac{1}{(z-w)^{3}} k^{(\mu} \partial X^{\nu)} \mathrm{e}^{i k \cdot X} \\
& +\frac{1}{(z-w)^{2}}\left(\frac{1}{2} k^{2}+1\right) \partial_{w} X^{\mu} \partial_{w} X^{\nu} \mathrm{e}^{i k \cdot X}+\frac{1}{z-w} \partial_{w}\left[\partial_{w} X^{\mu} \partial_{w} X^{\nu} \mathrm{e}^{i k \cdot X}\right] \\
& +(\text { finite as } z \rightarrow w) \\
T(z) V_{2}^{\mu}(w) \approx & \frac{1}{(z-w)^{4}} k^{\mu} \mathrm{e}^{i k \cdot X}+\frac{1}{(z-w)^{3}} \partial_{w} X^{\mu} \mathrm{e}^{i k \cdot X} \\
& +\frac{1}{(z-w)^{2}}\left(\frac{1}{2} k^{2}+1\right) \partial_{w}^{2} X^{\mu} \mathrm{e}^{i k \cdot X}+\frac{1}{z-w} \partial_{w}\left[\partial_{w}^{2} X^{\mu} \mathrm{e}^{i k \cdot X}\right] \\
& +(\text { finite as } z \rightarrow w)
\end{aligned}
$$

In the Hilbert space interpretation, the singularities in these operator products correspond to commutators of : $V_{1}^{\mu \nu}(z)$ : and $: V_{2}^{\mu}(z)$ : with $L_{n}=\oint(\mathrm{d} z / 2 \pi i) z^{n+1} T(z)$ for $n \geqslant-1$. Since the leading singularity is $(z-w)^{-4}$, only $L_{0}, L_{1}, L_{2}$ contribute. Physical state operators are constructed from linear combinations

$$
V_{\text {phys }}(k, z)=\zeta_{1}^{\mu \nu}(k) V_{1}^{\mu \nu}(k, z)+\zeta_{2}^{\mu}(k) V_{2}^{\mu}(k, z)
$$

such that the two leading singularities in (18) cancel to leave the operator product 
characteristic of $h=1$ highest weight vectors; hence

$$
\begin{gathered}
\eta_{\mu \nu} \zeta_{1}^{\mu \nu}+k_{\mu} \zeta_{2}^{\mu}(k)=0, \\
k_{\mu} \zeta_{1}^{\mu \nu}(k)+\zeta_{2}^{\mu}(k)=0, \\
k^{2}=-2 .
\end{gathered}
$$

The last of these reproduces the mass level condition; the first two are $d+1$ conditions on $\frac{1}{2} d(d+1)+d$ polarizations, leaving $\frac{1}{2}\left(d^{2}-d-2\right)$ physical state operators - a massive symmetric tensor multiplet; the $d$ combinations which are total derivatives $\partial\left(\partial X^{\mu} \mathrm{e}^{i k \cdot X}\right)$ won't contribute when integrated over the world sheet). The preceding construction clearly generalizes to any mass level $N$; the linear combinations of polarizations having finite operator products with $T(z)$ yield the physical vertices. The leading singularity will be of order $N+2$, so there are always only a finite number of constraints. Put differently, $L_{n}$ for $n>N$ automatically annihilates the states of level $N$ since it lowers the energy by $n$ units.

The vertex operators create asymptotic incoming states as $|z|=\mathrm{e}^{t} \rightarrow 0$; e.g.

$$
\mathrm{e}^{i k \cdot X(0)}|0\rangle=|k\rangle,
$$

the ground state of center of mass momentum $k$. Similarly,

$$
\begin{aligned}
\partial X^{\mu}(0)|0\rangle & =a_{-1}^{\mu}|0\rangle, \quad \partial^{2} X^{\mu}(0)|0\rangle=a_{-2}^{\mu}|0\rangle, \quad \text { etc. } \\
a_{n}^{\mu} & =\oint \frac{\mathrm{d} z}{2 \pi i} z^{n} \partial X^{\mu} .
\end{aligned}
$$

From these correspondences one can build up the operator which creates a given state in the Hilbert space. Information about the Hilbert space states can also be turned around to give the operator product coefficients by comparison with (13) evaluated as $z, w \rightarrow 0$. Having constructed the set of physical vertex operators, we may construct their correlation functions

$$
A(1 \ldots N)=\prod_{i=1}^{N} \int \mathrm{d}^{2} z_{i}\left\langle V_{1}\left(k_{1}, \zeta_{1}, z_{1}\right) \ldots V_{N}\left(k_{N}, \zeta_{N}, z_{n}\right)\right\rangle
$$

which are determined entirely in terms of the propagator (15). Unphysical states decouple from scattering processes as a result of conformal invariance [31,32]. The descendant fields (12) may be written as contour integrals about highest weight fields; the contour is then deformed away without encountering any singularities. The standard example is the amplitude for ground state scattering, where all the 
vertices in (19) are $V(k, z)=\mathrm{e}^{i k \cdot X}(z)$ with $k^{2}=2[41]$

$$
A_{K-N}(1 \ldots N)=\prod_{i=1}^{N} \int \mathrm{d}^{2} z_{i} \prod_{m<n} \exp \left[k_{m} \cdot k_{n} \log \left|z_{m}-z_{n}\right|^{2}\right] .
$$

The operator algebra (13) may be used to expose the resonances in (19) and (20) corresponding to on-shell intermediate states. For example, if $z_{i} \rightarrow z_{j}$ in (20),

$$
\begin{aligned}
\mathrm{e}^{i k_{i} X\left(z_{i}\right)} \mathrm{e}^{i k_{j} X\left(z_{j}\right) \approx} & \mathrm{e}^{k_{i} k_{j} \log \left|z_{i}-z_{j}\right|^{2}} \mathrm{e}^{i\left(k_{i}+k_{j}\right) X\left(z_{j}\right)} \\
& \times\left\{1+\left[\left(z_{i}-z_{j}\right)\left(k_{i}-k_{j}\right) \cdot \partial_{i} X+\text { c.c. }\right]\right. \\
& \left.\quad+\left|z_{i}-z_{j}\right|^{2}\left(k_{i}-k_{j}\right)^{\mu}\left(k_{i}-k_{j}\right)^{\nu} \partial_{i} X^{\mu} \partial_{i} X^{\prime \prime}+\cdots\right\}
\end{aligned}
$$

as $k_{i} \cdot k_{j} \rightarrow-1$, we have $\left(k_{i}+k_{j}\right)^{2} \rightarrow 2$ corresponding to an intermediate ground state tachyon resonance; $k_{i} \cdot k_{j} \sim-2$ picks out the massless pole $\left(k_{i}+k_{j}\right)^{2} \sim 0$, etc. Appropriate integrations by parts in the limit $z_{i} \rightarrow z_{j}$ pick out the relevant resonance; thus operator products factorize the amplitude. Poles only occur at physical states due to the decoupling theorems, so only the operator product coefficients of the ancestor fields enter. In particular the physical operators for higher mass levels can be deduced from any lower level operators carrying the relevant quantum numbers.

To summarize, the physical vertices of the bosonic string are in one-to-one correspondence with the dimension-one (in both $z$ and $\bar{z}$ ) conformal fields formed from combinations of $\partial X^{\mu}, \partial^{2} X^{\mu}$, etc., multiplied by $\mathrm{e}^{i k \cdot X}$ with $k^{2}=-m^{2}$, and such that the polarizations are physical. The states created by the conformal fields and their descendants span the Hilbert space. The operator products of the highest weight states of the Virasoro algebra factorize physical amplitudes. The descendant fields create the spurious states which fill out the representations of the Virasoro algebra. This structure has been used to explore classical string field theory [28].

The foregoing analysis of the physical state conditions may be recast in an equivalent BRST formulation [16,17]. The proper treatment of path integral measure factors associated with the gauge choice (2) has little impact on the calculation of tree-level string amplitudes since they contribute only to an overall normalization. On the other hand, string multiloop correlations will require Faddeev-Popov ghosts propagating in the loops in order to guarantee the decoupling of unphysical states. The modification is only in the measure for the bosonic string, as the ghosts decouple from the vertices. We will see below that this is no longer true for fermionic strings; the vertices for spacetime fermion emission couple to the ghosts.

The fluctuations about the gauge slice (2) are generated by infinitesimal diffeomorphisms [38, 42]

$$
\delta g_{z z}=\nabla_{z} \delta \xi_{z}, \quad \delta g_{\bar{z} \bar{z}}=\nabla_{\bar{z}} \delta \xi_{\bar{z}}
$$


The diffeomorphism group volume is factored out of the path integral by trading the integrations over the traceless metric fluctuations $\delta g_{z \bar{z}}, \delta g_{\bar{z} \bar{z}}$ for integrals over diffeomorphisms $\delta \xi_{z}, \delta \xi_{\bar{z}}$.

$$
\text { Q } g_{z z} \mathscr{D} g_{\bar{z} \bar{z}}=\operatorname{det} \nabla_{\bar{z}} \cdot \operatorname{det} \nabla_{\bar{z}} \mathscr{D} \xi_{z} \mathscr{D} \xi_{\bar{z}} \text {. }
$$

The determinants may be rewritten as a path integral over a conjugate pair of anticommuting Faddeev-Popov ghost fields $b_{z z}, c^{z}$ and $b_{\bar{z} \bar{z}}, c^{\bar{z}}$ with the action

$$
\begin{gathered}
S_{\text {ghost }}=\frac{1}{\pi} \int \mathrm{d}^{2} z\left[b_{z z} \partial_{\bar{z}} c^{z}+\text { c.c. }\right], \\
\partial_{\bar{z}} c^{z}=\partial_{\bar{z}} b_{z z}=0,
\end{gathered}
$$

imply that $b$ and $c$ are analytic fields. Hence the ghost system is also a conformal field theory. The fields $b, c$ are effectively free fermions of the wrong spin; the propagator on the plane is therefore

$$
\left\langle b_{z z} c^{w}\right\rangle=\frac{1}{z-w},
$$

with additional finite terms on higher genus surfaces. The variation of the covariant derivative on rank $n$ tensors under a traceless deformation of the metric is $[38,42]$

$$
\delta \nabla^{z}=\frac{1}{2} \delta g^{2 z} \nabla_{z}+\frac{1}{2} n \nabla_{z}\left(\delta g^{z z}\right) .
$$

Using this in the variation of (24), we find the traceless stress-energy tensor of the ghosts

$$
T_{\mathrm{gh}}(z)=c \partial b+2(\partial c) b .
$$

Using the two-point function (26) we may calculate the operator product

$$
T_{\mathrm{gh}}(z) T_{\mathrm{gh}}(w) \sim \frac{-13}{(z-w)^{4}}+\frac{2}{(z-w)^{2}} T_{\mathrm{gh}}(z)+\frac{1}{z-w} \partial T_{\mathrm{hh}}(z)+\text { nonsingular },
$$

demonstrating that $c=-26$ for this system. When the number of spacetime dimension $d=26$, the total anomaly $c_{x}+c_{\mathrm{gh}}$ vanishes and allows the consistent application of the Virasoro gauge conditions to decouple unphysical states. More properly, we should construct the BRST operator which is the gauge-fixed analogue of the reparametrization generators $T(z)$. If a field theory has a gauge symmetry with generators $G_{i}$, the general form of the BRST operator is [43]

$$
\begin{aligned}
Q_{\mathrm{BRST}} & =c^{i} G_{i}+\frac{1}{2} c^{i} c^{j} f_{i j}{ }^{k} \frac{\partial}{\partial c^{k}}, \\
{\left[G_{i}, G_{j}\right] } & =f_{i j}{ }^{k} G_{k} .
\end{aligned}
$$


$Q_{\mathrm{BRST}}$ is designed to satisfy $Q_{\mathrm{BRST}}^{2}=0$. In the case of the string, the conformal symmetry results in

$$
\begin{aligned}
j_{\mathrm{BRST}} & =c\left(T_{X}(z)+\frac{1}{2} T_{\mathrm{gh}}(z)\right)+\frac{3}{2} \partial^{2} c \\
& =-\frac{1}{2} c \partial X \partial X+b c \partial c+\frac{3}{2} \partial^{2} c, \\
Q_{\mathrm{BRST}} & =\oint \frac{\mathrm{d} z}{2 \pi i} j_{\mathrm{BRST}} .
\end{aligned}
$$

The reparametrization ghost $c^{z}$ is the parameter of BRST "gauge transformations"; the $\frac{1}{2}$ before $T_{\mathrm{gh}}$ ensures that $Q_{\mathrm{BRST}}$ generates the right transformation on the ghosts, and the last term is required in order that $j_{\mathrm{BRST}}$ is a conformal field (being a total derivative, it has no effect on flat space correlation functions, but ensures the conservation of the BRST current on curved world surfaces). The BRST charge acts on the fields as

$$
\begin{aligned}
{\left[Q_{\mathrm{BRST}}, O(z)\right] } & =\oint \frac{\mathrm{d} w}{2 \pi i} j_{\mathrm{BRST}}(w) O(z), \\
{\left[Q_{\mathrm{BRST}}, X^{\mu}\right] } & =c^{z} \partial_{z} X^{\mu}, \\
{\left[Q_{\mathrm{BRST}}, c^{z}\right]_{+} } & =c^{z} \partial_{z} c^{z}-\left(\partial_{z} c^{z}\right)^{2}=c^{z} \partial_{z} c^{2}, \\
{\left[Q_{\mathrm{BRST}}, b_{z z}\right]_{+} } & =T_{z z}[X]+c^{z} \partial_{z} b_{z z}+2\left(\partial_{z} c^{z}\right) b_{z z} \\
& =T_{z z}[X]+T_{z z}[b, c] .
\end{aligned}
$$

Again, the easiest way to calculate these commutators is to use operator products to evaluate contour integrals of the BRST current $j_{\text {BRST }}$ around $X, b$, and $c$. The conformal algebra and the cancellation of anomalies in $d=26$ translates into the statement

$$
Q_{\mathrm{BRST}}^{2}=0 \quad \text { for } d=26 .
$$

Because the ghosts are decoupled, physical states must be of the form (up to a null state)

$$
|\{n\} ; p\rangle_{X} \otimes c_{1}|0\rangle_{\mathrm{gh}}
$$

(here $\{n\} ; p$ denotes the occupation numbers and momentum of the state). The operator $c_{1}$ lowers the energy of the state by one unit and is necessary for BRST invariance. Since we can represent physical states in this way, only the part $\sum_{n \geqslant 0} c_{-n} L_{n}$ of $Q_{\text {BRST }}$ contributes to the BRST version of the physical state condition (17)

$$
\left.Q_{\mathrm{BRST}} \mid \text { phys }\right\rangle=0 .
$$


The ghost excitation in (32) may be thought of as responsible for the shift in the ground state energy which produces the tachyon (and thus the condition $L_{0}-1 \mid$ phys $\rangle_{X}=0$ ). Hence we see directly the equivalence of the BRST formalism and the operator methods of the past. The operator equivalent of (33) is

$$
\left[Q_{\mathrm{BRST}}, V_{\mathrm{phys}}\right]=0 \text {, }
$$

by which we mean that $Q_{\text {BRST }}$ commutes with $V_{\text {phys }}(z)$ up to total derivatives, which vanish when the vertex operator is integrated over the world sheet. Note that any operator of the form

$$
V_{\mathrm{phys}}=\left[Q_{\mathrm{BRST}}, O\right]
$$

where $O$ is any operator, is physical due to the Jacobi identity and (33); however such operators create states of the form $\mid$ null $\rangle=Q_{\text {BRST }} \mid$ anything $\rangle$ which have zero norm. Thus it is only the operators with nontrivial "BRST cohomology" that are of concern, and we may choose the states (32) to be the characteristic representatives of the cohomology classes.

The bosonic string BRST quantization procedure as described thus far differs little from the covariant operator formalism of the old dual model $[7,44]$. Its main advantage, which we now discuss, is the description of the vacuum of the string theory which it provokes. In particular, the ghost partition function provides the proper measure factor in multiloop amplitudes, a difficulty never fully resolved in the past [45].

In calculating the jacobian in (23) we should omit the zero modes of the operators $\nabla_{\bar{z}}$ and $\nabla_{z}$ [42]. These appear in the Faddeev-Popov procedure as nontrivial normalizable solutions of eqs. (25) in a background $\hat{\mathrm{g}}_{a b}$ appropriate to the world-sheet topology. The zero modes may be analyzed by treating the ghosts as fermions of the wrong spin (see sect. 5) coupled to a $U(1)$ gauge field, the $2 \mathrm{~d}$ spin connection. On the sphere, the gauge field is like a $U(1)$ instanton background. Because of these zero modes the ghost number current $j_{z}=c^{z} b_{z z}$ is anomalous [17]:

$$
\partial_{\bar{\Sigma}} j_{z}=\frac{1}{8} Q \sqrt{\hat{g}} R^{(2)}
$$

where $R^{(2)}$ is the intrinsic curvature of the world sheet described by $\hat{g}_{a b}$ and $Q=3$. The integrated anomaly yields an index theorem (the Riemann-Roch theorem [46]) for a surface with $g$ handles

$$
(\# \text { zero modes of } c)-(\# \text { zero modes of } b)=\frac{1}{2} Q \chi=Q(1-g) \text {. }
$$

Zero modes of $c$ are called conformal Killing vectors; $b$ zero modes are known as moduli, and correspond to the deformations of the metric $\hat{g}_{a b}$ which change its conformal structure. Further investigation shows $c$ has 3 zero modes for $g=0$ (the 
complexified isometries $\mathrm{SO}(3)$ of the 2-sphere), one complex zero mode for $g=1$ (the U(1) isometries of the torus), and none for $g \geqslant 2$. The index (35) then tells us that the sphere has no moduli; the torus has two moduli, one in $z$ and one in $\bar{z}$, describing the shape of the torus - its shear and elongation. For $g \geqslant 2$, the $3(g-1)$ complex moduli describe the "shape" of the surface of genus $g$. The path integral instructs us to integrate over all conformally inequivalent surfaces, thus the multiloop amplitudes will involve an integration over the moduli. The path integral provides the following prescription for the integration measure [38]: compute the full $b, c$ and $X^{\mu}$ Neumann functions for the surface in question (this will be detailed when we discuss fermionic strings). These propagators will involve the moduli as parameters, as will the correlation functions calculated using them. In particular, the partition function (1) is determined from

$$
\frac{\delta S_{\text {eff }}(\hat{g})}{\delta m_{i}}=\int\left[\frac{\delta g^{z z}}{\delta m_{i}} \frac{\delta S_{\text {eff }}}{\delta g^{z z}}+\text { c.c. }\right]=-\frac{1}{2} \int \frac{\mathrm{d}^{2} z}{2 \pi} \sqrt{\hat{g}} \hat{g}^{z \bar{z}}\left[\hat{g}_{\bar{z} z} \frac{\delta g^{z z}}{\delta m_{i}} T_{z z}^{\hat{g}}+\text { c.c. }\right]
$$

by integrating with respect to each $m_{i}, i=1, \ldots, 3(g-1)$, using the measure induced from the inner product

$$
\langle\delta g, \delta g\rangle=\int \mathrm{d}^{2} z \sqrt{\hat{g}}\left(\hat{g}^{a b} \hat{g}^{c d}-\frac{1}{2} \hat{g}^{a c} \hat{g}^{b d}\right) \delta g_{a c} \delta g_{b d} .
$$

The stress tensor $T_{z z}^{\hat{g}}$ is determined from the propagator

$$
T_{z z}^{\hat{\hat{g}}}=-\frac{1}{2}\left\langle\partial_{z} X^{\mu} \partial_{w} X^{\mu}\right\rangle_{z=w}+\left\langle c^{z} \partial_{w} b_{w w}+2\left(\partial_{z} c^{z}\right) b_{w w}\right\rangle_{z=w}
$$

in the coincidence limit $z \rightarrow w$. We subtract the leading singularity in the operator product expansion to render the expression well-defined; this is the analogue of normal ordering on a multiloop surface. The partition function $\mathrm{e}^{-S\left(\hat{g}\left(m_{t}\right)\right)}$ is the appropriate measure for modular integrals in $g$-loop amplitudes:

$$
\begin{aligned}
A(1, \ldots, N)= & \int \prod_{i=1}^{3(g-1)} \mathrm{d} \hat{g}\left(m^{i}\right) \mathrm{e}^{-S\left(\hat{g}\left(m_{j}\right)\right)} \times \prod_{k=1}^{N} \int \mathrm{d}^{2} z_{i} \sqrt{\hat{g}\left(z_{i}\right)} \cdot \prod_{l=1}^{3(g-1)} \int \mathrm{d}^{2} w_{l} \sqrt{\hat{g}\left(w_{l}\right)} \\
& \times\left\langle b_{1}\left(w_{1}\right) \ldots b_{3(g-1)}\left(w_{3(g-1)}\right) \cdot V_{1}\left(k_{1}, \zeta_{1}, z_{1}\right) \ldots V_{N}\left(k_{N}, \zeta_{N}, z_{N}\right)\right\rangle .
\end{aligned}
$$

The free-field expectation value is calculated with the relevant Neumann functions arising from the $X^{\mu}$ and $b, c$ path integrals; the $b_{l}\left(w_{l}\right)$ are zero-mode wave functions of $b_{z z}$ that are absorbed by the path integral over $b$. These ensure that the gauge fixing determinant is taken in the space orthogonal to the zero modes of 
the gauge fluctuation operator (22), and produce a factor in the measure of the determinant of the matrix of inner products of zero-mode wave functions [42].

Physical correlation functions only involve vertices satisfying (33), and therefore will only contain $X^{\mu}$. The demonstration that spurious states decouple involves an argument that we shall often invoke. The BRST charge is the contour integral of a dimension one analytic field. Spurious state operators are of the form $\left[Q_{\mathrm{BRST}}, O\right]$ for some $O$. This may be written as a contour integral of the BRST current about a contour circling $O$. When such a quantum field is inserted on a string world surface, perhaps in a correlation function with other vertices, the contour may be deformed away - pulled off the back of the surface, if you will. The BRST current only sees the analytic parts of the correlation functions, so there is no problem carrying out this operation. Specifically, any dimension $(1,0)$ or $(0,1)$ conformal field integrated along a contour is conformally invariant. In any coordinate patch one can deform the integration contour. Deforming the contour through other operators produces no commutator terms when the other operators are also BRST invariant. Eventually the contour sits in one patch with no singularity or operator inside, so the correlation function with the spurious state vertex vanishes. On multiply-handled world surfaces, the BRST contour does not pass through the $b$ zero modes in the correlation function (37), however the extra piece is a component of the stress energy tensor which is a total derivative with respect to a modulus of the surface (cf. eqs. (30), (36)); this contribution should vanish upon integration over the moduli space. This is the case when the moduli space is a closed manifold; however, in the example of open strings the boundary of the moduli space for the annulus as the inner hole shrinks away is the moduli space for the disk [47]. The discussion of BRST invariance on higher genus surfaces is really a rephrasing of the question of whether the theory is anomalous - i.e. do the longitudinal string modes decouple from physical processes? The bosonic string should not be problematic in this regard. The requirement of decoupling of BRST relics provides a check on the path integral measures for multiloop diagrams $[42,48]$ postulated on the basis of $2 \mathrm{~d}$ coordinate invariance. The contour deformation argument employed above is a useful tool in demonstrating several properties of the amplitudes, including some supersymmetry non-renormalization theorems (see sect. 6).

We must also consider the $c^{z}$ zero modes which enter into the structure of tree and one-loop amplitudes. The parameter space for tree amplitudes is the complex plane, on which the Laurent expansions of $c^{z}, b_{z z}$ are

$$
c(z)=\sum_{n} c_{n} z^{-n+1}, \quad b(z)=\sum_{n} b_{n} z^{-n-2} .
$$

The Fermi sea-level of the $b, c$ system determines the vacuum state $|0\rangle_{\mathrm{gh}}$ of the ghost Hilbert space. The canonical choice is the $\mathrm{SL}_{2}$ invariant state annihilated by the $L_{n}$ 
for $n=0, \pm 1$; inserting the expansions (38) into $T_{\mathrm{gh}}$ (eq. (28)) we find

$$
\begin{aligned}
& c_{n}|0\rangle_{\mathrm{gh}}=0, \quad n \geqslant 2, \\
& b_{n}|0\rangle_{\mathrm{gh}}=0, \quad n \geqslant-1 .
\end{aligned}
$$

Note that $|0\rangle_{\mathrm{gh}}$, although a highest weight state of the Virasoro algebra, is not a highest weight state of the $b, c$ algebra, i.e. it is not annihilated by all the negative frequency modes of $b$ and $c$ since

$$
c(0)|0\rangle_{\mathrm{gh}}=c_{1}|0\rangle_{\mathrm{gh}} \neq 0 .
$$

It is this state (or equivalently its degenerate partner $c \partial c(0)|0\rangle=c_{1} c_{0}|0\rangle$ ) which is the lowest energy state of the string Hilbert space, such that only positive frequency states propagate forward in time (or rather outward from the origin). The energy of this state

$$
L_{0}^{\text {tot }}\left(c(0)|0\rangle_{\mathrm{gh}} \otimes|0\rangle_{X}\right)=(-1) \cdot\left(c(0)|0\rangle_{\mathrm{gh}} \otimes|0\rangle_{X}\right)
$$

confirms that it produces the tachyonic vacuum of the bosonic string theory. Note that we never need to discuss divergent sums over zero-point energies to find the ground state energy - its value is a natural consequence of the conformal approach. Energies of states are always given by the dimensions of the operators which create them from the SL(2) invariant vacuum. The adjoint of this state is

$$
\left(c_{1}|0\rangle_{\mathrm{gh}}\right)^{\dagger}={ }_{\mathrm{gh}}\langle 0| c_{-1} c_{0} .
$$

This property is a consequence of the three zero modes of the $c$ field on the sphere:

$$
{ }_{\mathrm{gh}}\langle 0 \mid 0\rangle_{\mathrm{gh}}=0, \quad \text { but } \quad \mathrm{gh}_{\mathrm{gh}}\left\langle 0\left|c_{-1} c_{0} c_{1}\right| 0\right\rangle_{\mathrm{gh}}=1 .
$$

Indeed, in $Q_{\mathrm{BRST}}$ these modes multiply the $\mathrm{SL}_{2}$ generators $L_{0}, L_{ \pm 1}$ which act as conformal killing vectors on the fields. Because the correlation functions are invariant under the $\mathrm{SL}_{2}$ transformations (11) the amplitude is not completely gaugefixed. To factor out the $\mathrm{SL}_{2}$ group volume, we may use the three independent degrees of freedom in (11) to fix the location of any three vertices on the world sheet. This means that we drop three of the integrations in (19) and insert the jacobian factor

$$
\frac{\partial\left(z_{i}, z_{j}, z_{k}\right)}{\partial(a, b, c)}=\left|\left(z_{i}-z_{j}\right)\left(z_{i}-z_{k}\right)\left(z_{j}-z_{k}\right)\right|^{2}
$$

Alternatively, the desired result is a BRST invariant scattering amplitude. If $\int \mathrm{d}^{2} z V_{\text {phys }}$ 
is a physical vertex, then $c(z) \bar{c}(\bar{z}) V_{\text {phys }}(z)$ is a BRST invariant combination. One can use three such operators to cancel the background charge of the ghost system (i.e. to soak up the $c$ zero modes); because of the BRST invariance the location of the three $z$ 's is irrelevant, but any given choice removes the $\mathrm{SL}_{2}$ invariance (the $c$ correlations produce the factor (39)). Typically one chooses $z_{i}=(0,1, \infty)$; one can then think of the asymptotic "in" and "out" states as being built on the tachyonic ground state discussed above. Similar considerations apply on the torus. The upshot is that nonzero correlation functions in the ghost sector involve the zero-mode wave functions of $c$ as well as $b$, and one must fix the location of one vertex in scattering amplitudes.

This concludes our analysis of the conformal and BRST properties of the bosonic string. The use of the conformal algebra enabled us to describe the physical states of each mass level as highest weight vectors of the Virasoro algebra. The physical state conditions were then recast in a BRST formulation using the Faddeev-Popov ghosts of the conformal gauge. A proper treatment of the ghost system is essential to derive the correct integration measure for string loop amplitudes; a method was outlined whereby the measure factors could be derived from the field propagators and a parametrization of moduli space. Having completed this warm-up exercise, we now move on to discuss the fermionic string. Naturally, we begin with a description of the superconformal algebra and superconformal field theory.

\section{Superconformal field theory}

Superconformal field theory combines conformal field theory with supersymmetry. The rich structure of the Virasoro algebra admits two equally rich supersymmetric extensions, the Neveu-Schwarz (NS) [20] and Ramond (R) [19] algebras. These infinite algebras are generated by the moments of the super stress-energy tensor

$$
T(z, \theta)=T_{z \theta}(z)+\theta T_{z z}(z)
$$

which effect analytic coordinate and supersymmetry transformations on the local superfields $\phi_{j}(z, \theta ; \bar{z}, \bar{\theta})$ of the theory. Here $\theta, \bar{\theta}$ are complex analytic fermionic coordinates, the supersymmetry partners of $z, \bar{z}$ [49]. In this basis the two-dimensional $\gamma$-matrices are especially simple: $\gamma_{\theta \theta}^{z}=\gamma_{\bar{\theta} \overline{\bar{\theta}}}^{\overline{\bar{y}}}=1, \gamma_{\theta}^{3 \theta}=-\gamma_{\bar{\theta}}^{3 \bar{\theta}}=1$; indices are raised and lowered with $\varepsilon_{\theta \bar{\theta}}=-\varepsilon_{\bar{\theta} \theta}=1$, and all other components of these matrices vanish. Complex analysis has a simple extension to superspace [14,50]. There are complex superderivatives

$$
D=\partial_{\theta}+\theta \partial_{z}, \quad \bar{D}=\partial_{\bar{\theta}}+\bar{\theta} \partial_{\bar{z}},
$$

in terms of which the supersymmetry algebra is simply

$$
D^{2}=\partial_{z}, \quad \bar{D}^{2}=\partial_{\bar{z}} .
$$


We again focus on the analytic $(z, \theta)$ part to avoid repetition. Define the coordinate displacements

$$
z_{12}=z_{1}-z_{2}-\theta_{1} \theta_{2}, \quad \theta_{12}=\theta_{1}-\theta_{2},
$$

whose derivatives are

$$
\begin{aligned}
& D_{1} z_{12}=D_{2} z_{12}=\theta_{12}, \\
& D_{1} \theta_{12}=-D_{2} \theta_{12}=1 .
\end{aligned}
$$

These are useful for instance in writing the Taylor expansion of any superfunction about a point in superspace:

$$
\begin{aligned}
f\left(z_{1}, \theta_{1}\right) & =\sum_{n=0} \frac{1}{n !}\left(z_{12}\right)^{n}\left(\partial_{2}\right)^{n}\left[f\left(z_{2}, \theta_{2}\right)+\theta_{12} D_{2} f\left(z_{2}, \theta_{2}\right)\right] \\
& =f\left(z_{2}, \theta_{2}\right)+\theta_{12} D_{2} f\left(z_{2} \theta_{2}\right)+z_{12} \partial_{2} f+\cdots .
\end{aligned}
$$

Contour integration and Cauchy's theorem also have analogues: defining $\int \mathrm{d} \theta \theta=1$, $\int \mathrm{d} \theta 1=0$, we have

$$
\begin{aligned}
& \frac{1}{2 \pi i} \oint \mathrm{d} z_{1} \mathrm{~d} \theta_{1} f\left(z_{1}, \theta_{1}\right) \frac{\theta_{12}}{z_{12}^{n+1}}=\frac{1}{n !} \partial_{2}^{n} f\left(z_{2}, \theta_{2}\right), \\
& \frac{1}{2 \pi i} \oint \mathrm{d} z_{1} \mathrm{~d} \theta_{1} f\left(z_{1}, \theta_{1}\right) \frac{1}{z_{12}^{n+1}}=\frac{1}{n !} \partial_{2}^{n} D_{2} f\left(z_{2}, \theta_{2}\right) .
\end{aligned}
$$

An infinitesimal super vector field $V(z, \theta)=v_{0}+\theta v_{1}$ parametrizes infinitesimal supercoordinate transformations

$$
\begin{aligned}
& \delta z=v_{0}+\frac{1}{2} \theta v_{1}=V-\theta \delta \boldsymbol{\theta}, \\
& \delta \boldsymbol{\theta}=\frac{1}{2}\left(v_{1}+\theta \partial v_{0}\right)=\frac{1}{2} D V .
\end{aligned}
$$

This acts on conformal fields $\phi(z, \theta)$ of conformal weight $h$ as

$$
\delta \phi=L_{V} \phi=\left[V \partial+\frac{1}{2}(D V) D+h(\partial V)\right] \phi .
$$

The commutator of two such super-Lie derivatives is then

$$
\begin{aligned}
{\left[L_{V}, L_{W}\right] } & =L_{[V, W]}, \\
{[V, W] } & =V \partial W-W \partial V+\frac{1}{2}(D V)(D W) .
\end{aligned}
$$


As was mentioned above, the super stress-energy tensor (40) generates the transformation (43) by (anti) commutation relations which are now written as superspace contour integrals (cf. the derivation of eq. (7))

$$
L_{V} \phi=\frac{1}{2 \pi i} \oint \mathrm{d} z \mathrm{~d} \theta V \cdot T \cdot \phi
$$

The operator product which produces (43) using (41) is

$$
T\left(z_{1}, \theta_{1}\right) \phi\left(z_{2}, \theta_{2}\right) \sim h \frac{\theta_{12}}{z_{12}^{2}} \phi\left(z_{2}, \theta_{2}\right)+\frac{\frac{1}{2}}{z_{12}} D_{2} \phi+\frac{\theta_{12}}{z_{12}} \partial_{2} \phi \ldots,
$$

the superconformal algebra itself is

$$
T\left(z_{1}, \theta_{1}\right) T\left(z_{2}, \theta_{2}\right) \sim \frac{\frac{1}{4} \hat{c}}{z_{12}^{3}}+\frac{\frac{3}{2} \theta_{12}}{z_{12}^{2}} T\left(z_{2}, \theta_{2}\right)+\frac{\frac{1}{2}}{z_{12}} D_{2} T+\frac{\theta_{12}}{z_{12}} \partial_{2} T+\cdots
$$

In terms of the coefficients of the Laurent expansions

$$
\begin{aligned}
& T(z, \theta)=\sum_{n} z^{-n-3 / 2}\left(\frac{1}{2}\right) G_{n}+\theta \sum_{n} z^{-n-2} L_{n}, \\
& \phi(z, \theta)=\sum_{n} z^{-n-h} \phi_{0, n}+\theta \sum_{n} z^{-n-h-1 / 2} \phi_{1, n},
\end{aligned}
$$

the algebra becomes

$$
\begin{aligned}
& {\left[L_{m}, L_{n}\right]=(m-n) L_{m+n}+\frac{1}{8} \hat{c}\left(m^{3}-m\right) \delta_{m+n, 0},} \\
& {\left[G_{m}, G_{n}\right]_{+}=2 L_{m+n}+\frac{1}{2} \hat{c}\left(m^{2}-\frac{1}{4}\right) \delta_{m+n, 0}} \\
& {\left[L_{m}, G_{n}\right]=\left(\frac{1}{2} m-n\right) G_{m+n}}
\end{aligned}
$$

and the fields transform as

$$
\begin{aligned}
{\left[L_{m}, \phi_{0}(z)\right] } & =z^{m+2} \partial \phi_{0}+h(m+2) z^{m} \phi_{0}(z), \\
{\left[\varepsilon G_{m}, \phi_{0}(z)\right] } & =\varepsilon z^{m+1 / 2} \phi_{1}(z), \\
{\left[L_{m}, \phi_{1}(z)\right] } & =z^{m+2} \partial \phi_{0}+\left(h+\frac{1}{2}\right)(m+1) z^{m} \phi_{1}(z), \\
{\left[\varepsilon G_{m}, \phi_{1}(z)\right] } & =\varepsilon\left(z^{m+1 / 2} \partial \phi_{0}+2\left(m+\frac{1}{2}\right) h z^{m-1 / 2} \phi_{0}(z)\right) .
\end{aligned}
$$

We have normalized the central charge $\hat{c}=\frac{3}{2} c$ so that $\hat{c}=1$ for a free scalar 
superfield $X(z, \theta)=x(z)+\theta \psi(z)$ consisting of a free scalar field of $c=1$ and free Majorana fermion of $c=\frac{1}{2}$. $\varepsilon$ is a Grassmann parameter which keeps track of statistics.

The fermionic components of the conformal superfields $V, \phi$, and $T$ are allowed to be double-valued fields since they are two-dimensional spinors. Thus the Hilbert space of the theory divides into two subspaces: the Neveu-Schwarz subspace [20] in which $\phi_{\text {fermionic }}^{\mathrm{NS}}\left(\mathrm{e}^{2 \pi i} z\right)=+\phi_{\mathrm{fermionic}}^{\mathrm{NS}}(z)$ and the Ramond subspace [19] in which $\phi_{\mathrm{f}}^{\mathrm{R}}\left(\mathrm{e}^{2 \pi i} z\right)=-\phi_{\mathrm{f}}^{\mathrm{R}}(z)$. If we transfer to cylindrical coordinates $(z, \theta) \rightarrow\left(\log z, \theta z^{-1 / 2}\right)$ the rule (4) shows that $\phi_{\mathrm{f}}^{\mathrm{R}}$ is periodic on the cylinder; it is the natural fermionic superfield component on the cylinder. From the mode expansion (44) we discern that the indices of the $G_{n}$ are integral in the Ramond sector and half-integral in the Neveu-Schwarz sector. Indeed, the expression of supersymmetry in the NS sector is $G_{-1 / 2}^{2}=L_{-1}$, the translation generator on the plane; in the $\mathrm{R}$ sector it is $G_{0}^{2}=$ $L_{0}-\frac{1}{16} \hat{c}$, the translation generator on the cylinder. Supersymmetry is unbroken if and only if there are Ramond ground states with $G_{0}=0$, i.e. $h=\frac{1}{16} \hat{c}$. Since the vacuum $|0\rangle$ is the lowest energy state (with $h=0$ ) it will occur in the NS sector; it is invariant under the global superconformal group generated by $L_{0}, L_{ \pm 1}$, and $G_{ \pm 1 / 2}$. The conformal superfields $\phi_{j}(z, \theta)$ of weight $h_{j}$ create all the NS highest weight states $\left|h_{j}\right\rangle=\phi_{j}(0,0)|0\rangle$, annihilated by all of the lowering operators $G_{n}, L_{n}$ for $n>0$. However the superfields do not exhaust the set of fields in the theory; they cannot create states in the Ramond sector since the boundary condition on fermionic states remains unchanged. The states of the Ramond sector are created by conformal fields called spin fields. The operator product expansion of a spin field with the fermionic parts of the NS superfields is non-local (i.e., double-valued) in order to generate the correct Fourier series expansion of fermions in the Ramond sector; spin fields flip the boundary condition on the fermion fields between periodic and anti-periodic. A spin field may be represented as the endpoint of a branch cut in the fermion fields. One should not think that there are two sets of superfields, one for NS boundary conditions and one for R boundary conditions; rather, there is one superfield whose fermionic component has its Laurent expansion modified in the presence of a spin field. Since $G_{0}$ and $L_{0}$ commute these spin fields come in pairs $S^{ \pm}(z)$ such that

$$
\begin{aligned}
\left|h^{ \pm}\right\rangle & =S^{ \pm}(0)|0\rangle, \\
\left|h^{-}\right\rangle & =G_{0}\left|h^{+}\right\rangle, \\
\left(h-\frac{1}{16} \hat{c}\right)\left|h^{+}\right\rangle & =G_{0}\left|h^{-}\right\rangle
\end{aligned}
$$

or, in operator products,

$$
T_{z \theta}(z) S^{ \pm}(w) \sim \frac{1}{2} \frac{1}{(z-w)^{3 / 2}} a_{ \pm} S^{\mp}(w),
$$

with $a_{+}=1, a_{-}=h-\frac{1}{16} \hat{c}$. If $h=\frac{1}{16} \hat{c}$, global supersymmetry is unbroken in the Ramond sector. In this case the ground states need not be paired - we can drop the 
states $\left|h^{-}\right\rangle$; the supersymmetry index $[14,51] \operatorname{tr}(-)^{F}$, or some appropriate modification, counts the number of these unpaired ground states. The general state in the Ramond sector is reached from one of the R ground states (created from the NS vacuum by the spin fields of lowest dimension) by applying products of superfields. The operator product expansion of the fermionic part of a superfield with a spin field will be an expansion in half-integral powers of $(z-w)$, as in (48); thus in the $\mathrm{R}$ sector $\phi_{\mathrm{f}}(z)\left|h^{ \pm}\right\rangle$will have a Laurent expansion in half integer powers of $z$, whereas $\phi_{\mathrm{f}}(z)|0\rangle$ in the NS sector has an expansion in integer powers of $z$. The different boundary conditions on the cylinder can be thought of as being due to a "hidden" spin operator at $z=0$.

In the general superconformal field theory, the operator algebra has a form analogous to (13), with similar consequences for crossing symmetry, etc. Irreducible representations of the NS and $\mathrm{R}$ algebras are created from superfield and spin field highest weight states by applying combinations of raising operators $L_{-n}$ and $G_{-n}, n>0$. The superfields and the spin fields play complementary roles. Picture the operators of the superconformal field theory as $2 \times 2$ matrices acting on a two-vector consisting of the NS and R Hilbert spaces. The superfields of the theory are "diagonal", taking the NS sector into itself and the R sector into itself:

$$
\left(\begin{array}{c}
\left|N^{\prime}\right\rangle \\
\left|R^{\prime}\right\rangle
\end{array}\right)=\left(\begin{array}{l|l}
\phi & \\
\hline & \phi
\end{array}\right)\left(\begin{array}{c}
|N S\rangle \\
|R\rangle
\end{array}\right) .
$$

The spin fields are "off-diagonal", interpolating between the NS sector and the R sector:

$$
\left(\begin{array}{c}
\left|\mathrm{NS}^{\prime}\right\rangle \\
\left|\mathrm{R}^{\prime}\right\rangle
\end{array}\right)=\left(\begin{array}{l|l} 
& S \\
\hline S &
\end{array}\right)\left(\begin{array}{c}
|\mathrm{NS}\rangle \\
|\mathrm{R}\rangle
\end{array}\right) .
$$

The dimensions of the superfields are given by the energies of the NS states they create from the NS vacuum state; the dimensions of the spin fields are determined from the energies of the $R$ states they create from the NS vacuum state. The full superconformal field theory is not local since the fermionic fields are double-valued about the spin fields; in other words the operator product of $\phi_{\mathrm{f}}(z)$, the fermionic component of a superfield, with a spin field $S(w)$ is expanded in half integral powers of $(z-w)$. Locality is essential in order to have a well-defined string theory, where correlation functions must be integrated over their arguments - such integrals are ill-defined at the branch points of the correlations. It is difficult to have a Hilbert space interpretation when one requires information about the multivaluedness of the expectation values. There are two ways to project onto a local field theory, i.e. such that all operator products contain integer powers of $(z-w)$. The first is to restrict ourselves to one of the two fermion boundary conditions, NS or R, giving the usual algebra of superfields on the plane or the cylinder.

The second way, chosen by the string theory [10], takes both sectors; upon eliminating half of each, one regains a local field theory. The fermion parity operator 
$(-)^{\mathrm{F}} \equiv \Gamma$ anticommutes with all of the fermionic components of the superfields and commutes with the bosonic parts, counting fermion number mod2. Since it anticommutes with $T(z, \theta)$ (and the hamiltonian $L_{0}$ in particular), $\Gamma$ imposes a superselection rule on the theory. Because of its role in the fermionic string theory we refer to $\Gamma$ as the chirality operator. Witten's index [51] $\operatorname{tr}(-)^{\mathrm{F}}$ in the Ramond sector counts the number of chirally asymmetric (unpaired) $h=\frac{1}{16} \hat{c}$ Ramond ground states. If the index is nonzero, then $2 \mathrm{~d}$ supersymmetry cannot be broken, and some states will exist with $h=\frac{1}{16} \hat{c}$; otherwise $2 \mathrm{~d}$ supersymmetry is generally broken. Spin fields of opposite chirality are nonlocal with respect to each other since their operator product contains $2 \mathrm{~d}$ fermionic fields; spin fields of the same chirality are mutually local and their operator products close on bosonic fields, including fermion bilinears. Projection onto the $\Gamma=1$ subsector yields a local field theory, the spin model. The projected theory is local because $\phi_{\mathrm{f}}(z)$ changes the chirality but is projected out, so there are no square roots in operator products. Spin fields of opposite chirality recreate $\phi_{\mathrm{f}}$ in their operator product, so one chirality of the collection of $S$ 's must also be removed. The fields which remain are the bosonic parts of the superfields (including fermion bilinears) and the spin fields of positive chirality. This projection turns out to be required in the fermionic string theory in order that multiloop string amplitudes are invariant under the modular transformations mentioned in the previous section [52]; in fact, it is precisely this projection which yields the superstring [10].

\section{Fermionic strings}

\subsection{INTRODUCTION}

The fermionic string is obtained from the reparametrization invariant action (1) by incorporating local supersymmetry. Thus the string coordinates $X^{\mu}(\xi)$ acquire two-dimensional supersymmetric partners $\psi^{\mu}(\xi)$; a gravitino $\chi^{a}$ gauges the supersymmetry and, like $\sqrt{g} g^{a b}$, is a nondynamical Lagrange multiplier. The action $[29,8]$

$$
S=\int \mathrm{d}^{2} \xi \sqrt{g}\left[\frac{1}{2} g^{a b} \partial_{a} X^{\mu} \partial_{b} X^{\mu}+\frac{1}{2} i \psi^{\mu} \gamma^{a} \nabla_{a} \psi^{\mu}+\frac{1}{2} i\left(\chi_{a} \gamma^{b} \gamma^{a} \psi^{\mu}\right)\left(\partial_{b} X^{\mu}-\frac{1}{4} i \chi_{b} \psi^{\mu}\right)\right]
$$

is invariant under the local supersymmetry transformations

$$
\begin{aligned}
\delta g_{a b} & =2 i \varepsilon \gamma_{(a} \chi_{b)}, \\
\delta \chi_{a} & =2 \nabla_{a} \varepsilon, \\
\delta X^{\mu} & =i \varepsilon \psi^{\mu}, \\
\delta \psi^{\mu} & =\gamma^{a}\left(\partial_{a} X^{\mu}-\frac{1}{2} i \chi_{a} \psi^{\mu}\right) \varepsilon .
\end{aligned}
$$


Both gauge choices described for the bosonic string have supersymmetric analogues. We choose the covariant superconformal gauge $[29,8]$

$$
g_{a b}=\rho \delta_{a b}, \quad \chi_{a}=\gamma_{a} \zeta,
$$

which is the extension of (2). The action (49) now reduces to

$$
S_{\text {gauge-fixed }}=\int \mathrm{d}^{2} \xi\left[\frac{1}{2}\left(\partial_{a} X^{\mu}\right)^{2}-\frac{1}{2} i \psi^{\mu} \partial \psi^{\mu}\right] .
$$

The fields $\rho$ and $\zeta$ decouple due to the super-Weyl invariance of (49). The free fields $X^{\mu}, \psi^{\mu}$ may be combined into one free superfield $\boldsymbol{X}^{\mu}(z, \bar{z}, \theta, \bar{\theta})$ with the action

$$
S_{\text {gauge-fixed }}=\int \mathrm{d}^{2} z \mathrm{~d}^{2} \theta \frac{1}{2} \bar{D} \boldsymbol{X}^{\mu} D \boldsymbol{X}^{\mu} .
$$

The residual conformal invariance of (50) extends to superconformal invariance generated by

$$
\begin{aligned}
T(z, \theta) & =-\frac{1}{2} D \boldsymbol{X}^{\mu} D^{2} \boldsymbol{X}^{\mu} \\
& =-\frac{1}{2}[\psi \cdot \partial X+\theta(\partial X \cdot \partial X+\partial \psi \cdot \psi)] .
\end{aligned}
$$

The bosonic component of $T$ is the usual stress tensor; the fermionic component is the spin- $\frac{3}{2}$ Noether current for conformal supersymmetry which can be seen in the last term of (49). The local invariance is needed to decouple the negative metric states created by $\psi_{0}$. The solution to the free field equations of motion

$$
X^{\mu}=X^{\mu}(z)+X^{\mu}(\bar{z})+\theta \psi^{\mu}(z)+\bar{\theta} \bar{\psi}^{\mu}(\bar{z})
$$

separates into analytic and antianalytic superfields $\boldsymbol{X}^{\mu}(z, \theta)$ and $\overline{\boldsymbol{X}}^{\mu}(\bar{z}, \overline{\boldsymbol{\theta}})$. The two-point function on the $z$-plane is

$$
\left\langle\boldsymbol{X}^{\mu}\left(z_{1}, \boldsymbol{\theta}_{1}\right) \boldsymbol{X}^{\nu}\left(z_{2}, \theta_{2}\right)\right\rangle=-\boldsymbol{g}^{\mu \nu} \log z_{12} .
$$

Because of this factorization we may again focus without loss of generality on the $z, \theta$ dependence of the theory. The $\bar{z}, \bar{\theta}$ dependence may be copied directly, or the $\bar{z}$ portion of the theory may be taken to be a bosonic string theory as in the heterotic string [12]; we would then have a $(1,0)$ Majorana-Weyl supersymmetry on the world sheet, but our analysis of the analytic $z, \theta$ supersymmetry is unchanged. The reparametrization algebra of the superfields is

$$
T\left(z_{1}, \theta_{2}\right) D \boldsymbol{X}^{\mu}\left(z_{2}, \theta_{2}\right)-\frac{1}{2} \frac{\theta_{12}}{z_{12}} D_{2} \boldsymbol{X}^{\mu}+\frac{\frac{1}{2}}{z_{12}} D_{2}\left(D_{2}\left(\boldsymbol{X}^{\mu}\right)\right)+\frac{\theta_{12}}{z_{12}} \partial_{2}\left(D_{2} \boldsymbol{X}^{\mu}\right)
$$

and $T(z, \theta)$ satisfies (45) with $\hat{c}=d$. 
It is useful to examine the equivalent operator structure of $\boldsymbol{X}^{\mu}$. In the NS sector the conformal field $D X^{\mu}(z, \theta)$ may be expanded in mode operators

$$
D \boldsymbol{X}^{\mu}=\sum_{n \in \mathbb{Z}} z^{-n-1}\left[\psi_{n+1 / 2}^{\mu}+\theta a_{n}^{\mu}\right] .
$$

The field $\psi^{\mu}(z)$ has a half-integral mode expansion. These mode operators must be supplemented by the zero mode $q^{\mu}$ conjugate to $a_{0}^{\mu}$ in order to completely characterize $X^{\mu}(z, \theta)$. The super-SL $\mathrm{SL}_{2}$ invariant vacuum $|0\rangle_{\boldsymbol{X}}$, annihilated by all the $a_{n}^{\mu}$ and $\psi_{n}^{\mu}$ for $n>0$, resides in this sector. With $\mathrm{R}$ boundary conditions the fields $\psi^{\mu}(z)$ have integer mode expansions. From the (anti)commutation relations (valid in either sector)

$$
\begin{gathered}
{\left[a_{n}^{\mu}, a_{m}^{\nu}\right]=-g^{\mu \nu} m \delta_{m,-n},} \\
{\left[a_{n}^{\mu}, q^{\nu}\right]=-g^{\mu \nu} \delta_{n, 0},} \\
{\left[\psi_{n}^{\mu}, \psi_{m}^{\nu}\right]_{+}=-g^{\mu \nu} \delta_{m,-n},}
\end{gathered}
$$

we see that the zero modes $\psi_{0}^{\mu}$ of the $\mathrm{R}$ sector form a Clifford algebra. These commute with $L_{0}$ (see (46)) and therefore act on the R ground states, which must transform collectively as a spacetime Majorana spinor. From our general discussion of the previous section, there will be a set of spin fields $S_{\alpha}(z)$ which create these ground states from the NS vacuum and which thus transform as a spacetime spinor. The operator product expansion of $\psi^{\mu}(z)$ and $S_{\alpha}(0)$ is responsible for the change in the mode expansion of $\psi^{\mu}$. We will follow the gamma matrix conventions

$$
\left[\gamma_{\mu}, \gamma_{\nu}\right]_{+}=-g_{\mu \nu}, \quad \gamma_{\alpha \beta}^{\mu}=\gamma_{\beta \alpha}^{\mu} .
$$

From (47) we see that $S_{\alpha}$ has conformal weight $h=\frac{1}{16} \hat{c}=\frac{1}{16} d$; we will find below that $d=10$ is needed for spurious state decoupling.

The spin model obtained by projecting onto even fermion number $\Gamma=(-)^{\mathrm{F}}=1$ is the superstring [10]. The NS vacuum state is assigned parity $\Gamma=-1$ (the rationale for this, as well as the values of the ground state energies in the NS and R sectors, will be provided when we discuss the Faddeev-Popov ghosts), so it and all states created from it using even numbers of $\phi_{\mathrm{f}}$ operators will be projected out. The lowest energy states remaining in the NS sector are the states $\psi_{-1 / 2}^{\mu}|0\rangle=D X^{\mu}(0)|0\rangle$ describing a ten-dimensional massless vector. Because of eq. (47) and the fact that $\Gamma$ anticommutes with $\psi^{\mu}, \Gamma$ acts on spin fields by $\Gamma S_{\alpha} \Gamma=\gamma_{11} S_{\alpha}$, so in the R sector $(-)^{F}=1$ projects onto the positive chirality spinor states; the $R$ ground states with $\gamma_{11}=1$ form a Majorana-Weyl spinor which combine together with the NS vector states into a massless vector supermultiplet. We will see below by constructing the spacetime supersymmetry current that all the states of the spin model form tendimensional supermultiplets. 
Although manifestly Lorentz invariant, the action (49) is not manifestly spacetime supersymmetric. This should not bother us greatly because it is the spin model which we wish to consider. Spacetime supersymmetry is a property of the algebra of spin model fields, not the $2 \mathrm{~d}$ superfields. The spin model properties are not readily apparent in the $2 \mathrm{~d}$ superfield action.

Calculations of fermionic string scattering amplitudes involve computing correlation functions of spin fields $S_{\alpha}$ and fermions $\psi^{\mu}$. These are completely specified by their properties under $\operatorname{SO}(9,1)$ current algebra [53]. In this framework the fermion bilinears

$$
j^{\mu \nu}(z)=\psi^{\mu} \psi^{\nu}(z)
$$

are a representation of the generators of an $\mathrm{SO}(9,1)$ affine algebra defined by the relations (with level $k=1$ )

$$
\begin{aligned}
{\left[j_{n}^{\mu \nu}, j_{m}^{\lambda \sigma}\right] } & =f_{\rho \tau}^{\mu \nu, \lambda \sigma} j_{n+m}^{\rho \tau}+\frac{1}{2} k n \delta_{\lambda \boldsymbol{\sigma}}^{\mu \nu} \delta_{n,-m} \\
j_{n}^{\mu \nu} & =\oint \frac{\mathrm{d} z}{2 \pi i} z^{n} j^{\mu \nu}(z)
\end{aligned}
$$

The stress-energy tensor is determined from these currents $[54,35]$ via

$$
T_{\psi}=\frac{-1}{4(d-1)}: j^{\mu \nu} j_{\mu \nu}
$$

as may be easily verified through the use of the operator product expansion

$$
j^{\mu \nu}(z) j_{\mu \nu}(w) \sim \frac{-d(d-1)}{(z-w)^{2}}+2(d-1) \partial \psi \cdot \psi(z)
$$

(normal ordering subtracts the leading singularity). The current algebra defines a conformal field theory in which $\psi^{\mu}$ and $S_{\alpha}$ are the conformal fields of the vector and spinor representations, highest weight vectors of both the $\operatorname{SO}(9,1)$ current algebra and the Virasoro algebra. Note that the current algebra also has a representation structure similar to the Virasoro algebra - highest weight states annihilated by all the positive frequency modes of the currents $j(z)$ together with a tower of descendant states generated by the action of the negative frequency modes of $j(z)$ on the highest weight states. The property (56) provides a method for calculating correlation functions $[35,55]$. Consider the relation $L_{-1}=(1 /(d-1)) j_{-1}^{\mu \nu} M^{\mu \nu}$ where $M^{\mu \nu}=j_{0}^{\mu \nu}$ are the Lorentz generators in a particular representation. Conformal fields of the representation $i$ therefore satisfy

$$
\left[j_{-1}^{\mu \nu} M_{i}^{\mu \nu}-(d-1) L_{-1}\right] \phi_{i}=0
$$


which implies in correlation functions

$$
\left[(d-1) \frac{\partial}{\partial z_{i}}-\sum_{j=1}^{N} \frac{M_{i}^{\mu \nu} M_{j}^{\mu \nu}}{z_{i}-z_{j}}\right]\left\langle\phi_{1}\left(z_{1}\right) \ldots \phi_{N}\left(z_{N}\right)\right\rangle=0
$$

since $L_{-1}$ generates translations and $j_{-1}$ group transformations on the fields. These linear matrix differential equations may then be solved for the correlations; the correlation function of four $S_{\alpha}$ 's has been calculated this way and agrees with previous results $[53,56]$. The method demonstrates that only the current algebra properties

$$
\begin{aligned}
& j^{\mu \nu}(z) \psi^{\lambda}(w) \sim \frac{1}{z-w}\left[g^{\mu \lambda} \psi^{\nu}(w)-g^{\nu \lambda} \psi^{\mu}(w)\right], \\
& j^{\mu \nu}(z) S_{\alpha}(w) \sim \frac{1}{z-w}\left(\psi^{[\mu} \gamma^{\nu]}\right)_{\alpha}^{\beta} S_{\beta}(w)
\end{aligned}
$$

are needed to determine all the spin field and superfield fermion correlations. In particular we find the operator algebra

$$
\begin{aligned}
& \psi^{\mu}(z) S_{\alpha}(w) \sim(z-w)^{-1 / 2} \gamma_{\alpha \beta}^{\mu} S^{\beta}(w)+\cdots, \\
& S^{\alpha}(z) S_{\beta}(w) \sim(z-w)^{-5 / 4} \delta_{\beta}^{\alpha}+(z-w)^{-1 / 4}\left(\frac{1}{2} \gamma^{\mu} \gamma^{\nu}\right)_{\beta}^{\alpha} \psi^{\mu} \psi^{\nu}+\cdots, \\
& S_{\alpha}(z) S_{\beta}(w)-(z-w)^{-3 / 4} \gamma_{\alpha \beta}^{\mu} \psi_{\mu}+\cdots,
\end{aligned}
$$

which shows that the $\psi^{\mu}$ 's act as generalized $\gamma$ matrices in the $\mathrm{R}$ sector and are indeed double-valued with respect to the spin fields. The first of (59) may be seen from (58) and $j_{\mu v} \propto \psi_{\mu} \psi_{v}$; the second equation is a consequence of spinor algebra and the dimension $h=\frac{5}{8}$ of $S_{\alpha}$. None of the operator relations (59) is single-valued in the $z$-plane, so string vertices will contain other fields in combination with $S_{\alpha}$ such that the correlation functions of them together are local.

The above relations are all group theoretic and independent of the relation (54), which is just a particular way of writing the currents in terms of the conformal fields of the vector representation. In fact, below we will make use of a different representation of the current algebra obtained by bosonization of the Wick rotated $\mathrm{SO}(10)$ currents.

SO(10) current algebra may also be realized on a set of five free bosons $H_{i}$ which parametrize the maximal torus of $\mathrm{SO}(10)$ [57]. This representation is obtained by choosing a maximal set of commuting generators - say $j_{2 i, 2 i+1}, i=0 \ldots 4$. These currents are then bosonized in the standard fashion [58]

$$
j_{2 i, 2 i+1}=\partial H_{i} .
$$


Since $\partial H_{i}$ are the momenta conjugate to $H_{i}$, the $H_{i}$ 's parametrize the maximal torus of $\mathrm{SO}(10)$ generated by these $\mathrm{U}(1)$ 's. The remaining 40 currents are realized nonlinearly as the exponentials

$$
\mathrm{e}^{i \alpha \cdot H}=: \mathrm{e}^{ \pm i\left(H_{i}+H_{j}\right)}: c_{ \pm i \pm j}, \quad i \neq j,
$$

of conformal weight 1 . The operators $c_{+i+j}$ generate a two-cocycle in commutation relations such that the algebra (55) is realized [57]. The vectors $\alpha$ denote the $\mathrm{U}(1)$ charges of the generators and are called the root vectors of the algebra. In fact the entire lattice of points

$$
\Lambda_{\mathrm{SO}(10)}=\left\{n_{i} \boldsymbol{e}_{i} \mid \text { all } n_{i} \in \mathbb{Z} \text { or all } n_{i} \in \mathbb{Z}+\frac{1}{2}, \boldsymbol{e}_{i} \cdot \boldsymbol{e}_{j}=\boldsymbol{\delta}_{i j}\right\}
$$

is the weight lattice of $\operatorname{SO}(10)$, such that $\mathrm{e}^{i \alpha \cdot H}$ with $\alpha \in \Lambda_{\mathrm{SO}(10)}$ lies in an $\operatorname{SO}(10)$ representation. For instance,

$$
\begin{gathered}
\psi^{2 j} \pm \psi^{2 j-1}=\mathrm{e}^{ \pm i H_{j}}, \\
S_{\alpha}=\prod_{j=0}^{4} \mathrm{e}^{ \pm i H_{i} / 2}
\end{gathered}
$$

are the dimension- $\frac{1}{2}$ vector representation and dimension- $\frac{5}{8}$ spinor representation conformal fields. Here the 32 spinor components are displayed in a helicity basis [59] specifying their spin projections on the five two-dimensional planes $\boldsymbol{e}_{2 i} \otimes \boldsymbol{e}_{2 i+1}$; the $\gamma_{11}$ helicity eigenvalue of a spinor is given by the product of all five signs in the exponent. Note that the product of two spinors indeed reproduces the currents (54). The advantage of the representation is that all correlation functions and operator products are given in terms of free field expressions such as (21). This "vertex representation" provides an explicit solution to the differential equation (57) in terms of the Koba-Nielsen amplitude (20). Moreover in contrast to the fermionic representation in terms of the $\psi^{\mu}$ 's we have a convenient way of handling the spin fields $S_{\alpha}$. Lastly, the projection $\Gamma=1$ removes the ground state and one of the spinor representations as well as any state reached from these by the tensor product of an even number of vectors, or from the remaining spinor by an odd number of vectors.

This completes our introduction to the spacetime fields of the fermionic string; the construction of string emission vertices and correlation functions will be undertaken once we have discussed the quantization of the Faddeev-Popov ghosts of the superconformal gauge (50). We shall see that the ghosts enter intrinsically into the fermion emission vertex and supersymmetry algebra. The operators needed to complete (59) to a local set of operator products will come from this sector. 


\section{Superconformal ghosts and the fermion vertex}

\subsection{GHOST FIELD THEORY AND SUPERCONFORMAL BRST FORMALISM}

As in the bosonic string, the Faddeev-Popov (super)determinant compensates for fixing the intrinsic supermetric on the world surface of the fermionic string. This superdeterminant is the jacobian for the change of variables

$$
\begin{aligned}
\delta g_{z z} & =\nabla_{z} \delta \xi_{z}, \\
\delta \chi_{z} & =\nabla_{z} \delta \varepsilon \quad \text { (plus c.c. equations) }
\end{aligned}
$$

used to factor out the super-reparametrization group [60]. The determinant of the differential operators in (61) may be represented by a path integral over a conjugate pair of dimension $-1, \frac{3}{2}$ ghost superfields

$$
C^{z}=c^{z}+\theta \gamma^{\theta}, \quad B_{z \theta}=\beta_{z \theta}+\theta b_{z z},
$$

whose action is

$$
S_{\text {ghost }}=\int \mathrm{d}^{2} z \mathrm{~d}^{2} \theta B_{z \theta} \bar{D} C^{z}
$$

We recognize $b, c$ as the reparametrization ghosts; the new feature is $\beta$ and $\gamma$, the commuting spinor ghosts of local supersymmetry. The spinor ghost system $\beta, \gamma$ is a first order lagrangian for Bose fields, hence its spectrum is unbounded below and one expects a catastrophe. We defer a discussion of the associated difficulties until the next subsection.

The equations of motion

$$
\bar{D} B_{z \theta}=\bar{D} C^{z}=0
$$

again show that the ghost fields are analytic superfunctions, with $z$-plane propagator

$$
\left\langle B\left(z_{1}, \theta_{1}\right) C\left(z_{2}, \theta_{2}\right)\right\rangle=\frac{\theta_{12}}{z_{12}} .
$$

In components we find that both $b, c$ and $\beta, \gamma$ have Dirac propagators $\left(z_{1}-z_{2}\right)^{-1}$. The super stress-energy tensor is most easily constructed in components, using (27) to obtain the stress-energy tensor and then converting the expression to superfields to find

$$
\begin{aligned}
T_{\mathrm{gh}}\left(z_{1} \theta\right) & =-C\left(D^{2} B\right)+\frac{1}{2}(D C)(D B)-\frac{3}{2}\left(D^{2} C\right) B \\
& =-c \partial \beta+\frac{1}{2} \gamma b-\frac{3}{2}(\partial c) \beta+\theta\left[c \partial b+2(\partial c) b-\frac{1}{2} \gamma \partial \beta-\frac{3}{2}(\partial \gamma) \beta\right] .
\end{aligned}
$$


The conformal properties of the ghost system are contained in

$$
\begin{aligned}
& T_{\mathrm{gh}}\left(z_{1}, \theta_{1}\right) C\left(z_{2}, \theta_{2}\right) \sim(-1) \cdot \frac{\theta_{12}}{z_{12}} C\left(z_{2}, \theta_{2}\right)+\frac{\frac{1}{2}}{z_{12}} D_{2} C+\frac{\theta_{12}}{z_{12}} \partial_{2} C \\
& T_{\mathrm{gh}}\left(z_{1}, \theta_{1}\right) B\left(z_{2}, \theta_{2}\right) \sim \frac{3}{2} \frac{\theta_{12}}{z_{12}} B\left(z_{2}, \theta_{2}\right)+\frac{\frac{1}{2}}{z_{12}} D_{2} B+\frac{\theta_{12}}{z_{12}} \partial^{2} B \\
& T_{\mathrm{gh}}\left(z_{1}, \theta_{1}\right) T_{\mathrm{gh}}\left(z_{2}, \theta_{2}\right) \sim(-10) \cdot \frac{\frac{1}{4}}{z_{12}}+\frac{3}{2} \frac{\theta_{12}}{z_{12}} T\left(z_{2}, \theta_{2}\right)+\frac{\frac{1}{2}}{z_{12}} D_{2} T+\frac{\theta_{12}}{z_{12}} \partial_{2} T .
\end{aligned}
$$

The last of these shows that the anomaly of the ghost + matter system is $\hat{c}=d-10$ which vanishes in $d=10$; the combined super conformal invariance of the ghostmatter system allows a proof of spurious state decoupling in string interactions [32].

We might remark at this point that the supersymmetric ghost system actually has a larger symmetry than might be expected a priori on the basis of the action (49) - a hidden $N=2$ superconformal invariance. In mode operators, the $N=2$ superconformal algebra is

$$
\begin{aligned}
{\left[L_{m}, L_{n}\right] } & =(m-n) L_{m+n}+\frac{1}{8} \hat{c}\left(m^{3}-m\right) \delta_{m,-n}, \\
{\left[L_{m}, G_{n}^{+}\right] } & =\left(\frac{1}{2} m-n\right) G_{m+n}^{ \pm}, \\
{\left[G_{m}^{+}, G_{n}^{+}\right]_{+} } & =0=\left[G_{m}^{-}, G_{n}^{-}\right]_{+}, \\
{\left[G_{m}^{+}, G_{n}^{-}\right]_{+} } & =L_{m+n}+\frac{1}{2}(m-n) H_{m+n}+\frac{1}{4} \hat{c}\left(m^{2}-\frac{1}{4}\right) \delta_{m,-n}, \\
{\left[H_{m}, G_{n}^{ \pm}\right] } & = \pm G_{m+n}^{ \pm}, \\
{\left[H_{m}, H_{n}\right] } & =\frac{1}{2} \hat{c} m \delta_{m,-n} .
\end{aligned}
$$

These relations show that $H(z)$ is a $\mathrm{U}(1)$ current (not to be confused with the $\mathrm{SO}(10)$ currents of the previous section) which rotates the two supercurrents $T_{\mathrm{F}}^{ \pm}(z)$. The $N=1$ supercurrent of eq. (40) is $T_{\mathrm{F}}(z)=T_{\mathrm{F}}^{+}(z)+T_{\mathrm{F}}^{-}(z)$; the other linear combination combines with $H(z)$ to form a dimension-one superfield

$$
j(z, \theta)=H(z)+\theta\left(T_{\mathrm{F}}^{+}(z)-T_{\mathrm{F}}^{-}(z)\right) .
$$

For the ghost system, the $N=2$ algebra is generated by (64) together with

$$
j(z, \theta)=2(D B) C+3 B(D C),
$$


which one readily verifies as satisfying (66). The significance of this observation has yet to be determined.

The super-BRST operator is once again given by the line integral of the current

$$
\begin{aligned}
j_{\mathrm{BRST}} & =C^{z}\left(T_{\text {matter }}(z, \theta)+\frac{1}{2} T_{\mathrm{gh}}(z, \theta)\right)-\frac{3}{4} D(C(D C) B), \\
Q_{\mathrm{BRST}} & =\oint \frac{\mathrm{d} z \mathrm{~d} \theta}{2 \pi i} j_{\mathrm{BRST}} \\
& =\oint \frac{\mathrm{d} z \mathrm{~d} \theta}{2 \pi i}\left(C T_{\text {matter }}-\delta C \cdot B\right),
\end{aligned}
$$

where

$$
\delta C=C \partial C-\frac{1}{4}(D C)(D C)
$$

From the two-point functions (52) and (63), as well as the operator products (53) and (65), we may determine the BRST transformation properties of all the NS superfields:

$$
\begin{aligned}
{\left[\varepsilon Q_{\mathrm{BRST}}, C\right] } & =\varepsilon \delta C, \\
{\left[\varepsilon Q_{\mathrm{BRST}}, B\right] } & =-\varepsilon T_{\text {matter }}, \\
{\left[\varepsilon Q_{\mathrm{BRST}}, \phi_{\text {matter }}\right] } & =\left[(\varepsilon C) \partial+\frac{1}{2} D(\varepsilon C) D+h \partial(\varepsilon C)\right] \phi_{\text {matter }},
\end{aligned}
$$

where $\varepsilon$ is an anticommuting parameter and $\phi_{\text {matter }}$ is a dimension- $h$ superconformal field of the string coordinates. The superconformal algebra of the stress tensors (51) plus (64) translates into the BRST relation (for $d=10$ only)

$$
Q_{\mathrm{BRST}}^{2}=0 .
$$

The physical vertices of both NS and R sectors must satisfy

$$
\left[\varepsilon Q_{\text {BRST }}, V_{\text {phys }}\right]=0 .
$$

Of course an identical statement applies to the BRST charge $\bar{Q}_{\text {BRST }}$ of the antianalytic conformal algebra. In the NS sector, vertex operators which are highest weight vectors of the $X, \psi$ superconformal algebras generated by $T_{\text {matter }}(z, \theta), \bar{T}_{\text {matter }}(\bar{z}, \bar{\theta})$ satisfy (69) (and of course the corresponding statement with $\bar{Q}_{\text {BRST }}$ ). We see that the integrals of such operators over superspace will be BRST invariant if they carry conformal weight $h=\left(\frac{1}{2}, \frac{1}{2}\right)$ :

$$
V_{\text {phys }}=\int \mathrm{d}^{2} z \mathrm{~d}^{2} \theta V_{h=1 / 2}(z, \theta) \bar{V}_{\bar{h}=1 / 2}(\bar{z}, \bar{\theta}) .
$$


For example, from the highest weight superfield

$$
V_{\mathrm{B}}=D X^{\mu} \mathrm{e}^{i k \cdot x}(z, \theta)
$$

we can build the massless vertices for emission of gravitons, dilatons, and antisymmetric tensors

$$
V_{\text {massless }}(k, \zeta)=\int \mathrm{d}^{2} z \mathrm{~d}^{2} \theta \zeta_{\mu \nu}(k) D X^{\mu} \bar{D} X^{\nu} \mathrm{e}^{i k \cdot X},
$$

with $h=\bar{h}=\frac{1}{2} k^{2}+\frac{1}{2}=\frac{1}{2}$, and $k^{\mu} \zeta_{\mu \nu}=k^{\nu} \zeta_{\mu \nu}=0$ in order that the operator is a highest weight vector. The spin and symmetry of the polarization tensor $\zeta_{\mu \nu}$ determines which type of particle is emitted from the string. Note that these vertices commute with $\Gamma=(-)^{\mathrm{F}}$ and $\bar{\Gamma}=(-)^{\overline{\mathrm{F}}}$ separately. On the other hand the tachyon vertex

$$
V_{\text {tachyon }}=\int \mathrm{d}^{2} z \mathrm{~d}^{2} \theta \mathrm{e}^{i k \cdot X}, \quad k^{2}=1,
$$

has $\Gamma V_{\text {tachyon }} \Gamma=-V_{\text {tachyon }}$ since $\Gamma$ anticommutes with $\theta$. Thus the tachyon is projected out upon passing to the superstring, as it must be - there is no scalar supermultiplet in $d=10$. For the heterotic string, similar considerations apply, but the antianalytic supersymmetry is absent $-\bar{\theta}$ is absent, and $\bar{D}$ is replaced by $\bar{\partial}$. There are of course other massless string vertices associated with vector gauge bosons.

The vertices (70) together with those obtained by factorization using operator products (i.e. all the other BRST-invariant states), provide a complete description of the bosonic (NS) sector of the superstring. To complete the picture we would like to find the fermion (R) vertex operators. The analysis simplifies if we separate $Q_{\text {BRST }}$ into three component field pieces characterized by their spinor ghost charges:

$$
\begin{aligned}
Q_{\mathrm{BRST}} & =Q_{0}+Q_{1}+Q_{2}, \\
Q_{0} & =\oint \frac{\mathrm{d} z}{2 \pi i}\left(c \tilde{T}_{\mathrm{B}}(X, \psi ; \beta, \gamma)-b c \partial c\right), \\
Q_{1} & =\oint \frac{\mathrm{d} z}{2 \pi i} \frac{1}{2} \gamma \psi^{\mu} \partial X^{\mu}, \\
Q_{2} & =\oint \frac{\mathrm{d} z}{2 \pi i} \frac{1}{4} \gamma^{2} b .
\end{aligned}
$$

Here $\tilde{T}_{\mathrm{B}}(x, \psi ; \beta, \gamma)$ is the combined stress tensor of all the fields mentioned; thus $Q_{0}$ is the bosonic string BRST operator (cf. eq. (29)), as if $\beta$ and $\gamma$ were extra matter fields. $Q_{1}$ is the world-sheet supersymmetry generator of the string coordinates 
multiplied by the corresponding (commuting) ghost parameter of local supersymmetry. $Q_{2}$ is needed for closure of the algebra; its source is the ghost supercurrent.

We are now prepared to investigate the requirements to be met by physical fermion vertices. Because these operators will create Ramond states from the Neveu-Schwarz vacuum they will be constructed out of spin fields, not superfields. The reparametrization constraint implied by $Q_{0}$ is again that the integral $\int \mathrm{d}^{2} z V(z, \bar{z})$ of the vertex operator over the world sheet be conformally invariant, which is guaranteed if the operator is dimension $(1,1)$. In addition, the operator product $(59)$ shows that $\psi^{\mu}$ acts on the spin fields as a generalized Dirac matrix. Thus $Q_{1}$ acts on fermion vertices as a generalized Dirac operator (the Dirac-Ramond operator [19]), so the fermion vertices must satisfy the Dirac equation. The above considerations dictate that the analytic part of the vertex for massless fermion emission must have the general structure $u^{\alpha}(k) \cdot \Sigma S_{\alpha} \mathrm{e}^{i k \cdot x}$, with $k^{2}=k u=0 ; \Sigma$ is some dimension- $\frac{3}{8}$ operator whose presence is needed because $S_{\alpha}$ only has dimension $\frac{5}{8}$. It is also needed to make the operator algebra local. Our survey of $\mathrm{SO}(9,1)$ current algebra yielded no operators of that dimension, so we must look elsewhere. Goddard and Olive suggested that such an operator might come from the ghost sector of the theory. The line of reasoning is as follows: the original action (49) couples the gravitino $\chi_{a}$ to the spinor $\psi^{\mu}$, thus $\chi_{a}$ (and also the parameter $\varepsilon$ of local supersymmetry variations) must obey the same boundary conditions as $\psi^{\mu}$ in order that the action is well-defined and locally supersymmetric. In the neighborhood of a spin field, $\psi^{\mu}$ is double-valued and consequently so are $\chi_{a}$ and $\varepsilon$.

The Faddeev-Popov ghost system which represents the gauge-fixing determinant will not completely decouple from the theory because the variational operator (61) has its boundary conditions changed at the location of the spin field $S_{\alpha}$, i.e., the ghost fields $\beta, \gamma$ reflect the boundary conditions of $\chi_{a}, \varepsilon$. The required operator $\Sigma$ is just the spin operator of the spinor ghost system. In the case of the spacetime fields the properties of the spin fields were discovered through their operator products under $\mathrm{SO}(9,1)$ current algebra. We will see that the ghost spin field $\Sigma$ is similarly determined its properties under the algebra of the ghost current $j_{\mathrm{gh}}=-\beta \gamma$. Thus we turn away from our development of string vertex operators to discuss in detail first order field theories such as (62), their currents, zero modes, stress tensors, and vacua.

\subsection{FIRST-ORDER LAGRANGIANS IN 2d FIELD THEORY}

Consider the action

$$
S=\frac{1}{\pi} \int \mathrm{d}^{2} z(\boldsymbol{b} \bar{\partial} \boldsymbol{c})
$$

where $\boldsymbol{b}$ and $\boldsymbol{c}$ denote general conjugate fields of dimension $\lambda$ and $1-\lambda$ respectively; they can be either Bose or Fermi fields - we treat both cases in parallel. The ghosts $b, c$ and $\beta, \gamma$ of previous sections are just special cases. The $\boldsymbol{b}, \boldsymbol{c}$ operator 
product is

$$
\begin{gathered}
\boldsymbol{c}(z) \boldsymbol{b}(w) \sim \frac{1}{z-w}, \\
\boldsymbol{b}(z) \boldsymbol{c}(w) \sim \frac{\varepsilon}{z-w} .
\end{gathered}
$$

Here and in the sequel, $\varepsilon=+1$ for Fermi statistics and $\varepsilon=-1$ for Bose statistics. The fields have the mode expansions and hermiticity properties

$$
\begin{array}{lll}
\boldsymbol{c}(z)=\sum_{n} z^{-n-(1-\lambda)} \boldsymbol{c}_{n}, & \boldsymbol{c}_{n}^{\dagger}=\boldsymbol{c}_{-n}, \\
\boldsymbol{b}(z)=\sum_{n} z^{-n-\lambda} \boldsymbol{b}_{n}, & \boldsymbol{b}_{n}^{\dagger}=\boldsymbol{\varepsilon} \boldsymbol{b}_{-n}
\end{array}
$$

and the operator product determines the (anti) commutation relations

$$
\boldsymbol{c}_{m} \boldsymbol{b}_{n}+\varepsilon \boldsymbol{b}_{n} \boldsymbol{c}_{m}=\delta_{m,-n} .
$$

There are NS and R sectors of the theory, specified by

$$
\begin{array}{lll}
\text { NS: } & \boldsymbol{b}_{n}, & n \in \mathbb{Z}-\lambda, \\
& \boldsymbol{c}_{n}, & n \in \mathbb{Z}+\lambda, \\
\mathrm{R}: & \boldsymbol{b}_{n}, & n \in \frac{1}{2}+\mathbb{Z}-\lambda, \\
& \boldsymbol{c}_{n}, & n \in \frac{1}{2}+\mathbb{Z}+\lambda .
\end{array}
$$

The stress tensor (cf. (64)) and reparametrization algebra are

$$
\begin{gathered}
T=-\lambda \boldsymbol{b} \partial \boldsymbol{c}+(1-\lambda)(\partial \boldsymbol{b}) \boldsymbol{c} \\
=\frac{1}{2}[(\partial \boldsymbol{b}) \boldsymbol{c}-\boldsymbol{b} \partial \boldsymbol{c}]+\left(\frac{1}{2}-\lambda\right) \partial(\boldsymbol{b} \boldsymbol{c}), \\
T(z) \boldsymbol{b}(w) \sim \frac{\lambda}{(z-w)^{2}} \boldsymbol{b}(w)+\frac{1}{z-w} \partial_{w} \boldsymbol{b}, \\
T(z) \boldsymbol{c}(w) \sim \frac{1-\lambda}{(z-w)^{2}} c(w)+\frac{1}{z-w} \partial_{w} c, \\
T(z) T(w)-\frac{-\varepsilon\left(6 \lambda^{2}-6 \lambda+1\right)}{(z-w)^{4}}+\cdots, \\
c=-2 \varepsilon\left(6 \lambda^{2}-6 \lambda+1\right)=\varepsilon\left(1-3 Q^{2}\right), \\
Q=\varepsilon(1-2 \lambda) .
\end{gathered}
$$


Special cases are the reparametrization ghost algebra with $\varepsilon=1, \lambda=2, Q=-3$ and $c=-26$; and the local supersymmetry ghost algebra with $\varepsilon=-1, \lambda=\frac{3}{2}, Q=2$ and $c=11$.

Another aspect of the linear system will be important to us: it has a $U(1)$ number current

$$
\begin{gathered}
j=-\boldsymbol{b} \boldsymbol{c}=\sum_{n} z^{-n-1} j_{n}, \\
j(z) \boldsymbol{b}(w) \sim \frac{-\boldsymbol{b}(w)}{z-w}, \\
j(z) \boldsymbol{c}(w) \sim \frac{\boldsymbol{c}(w)}{z-w}, \\
j(z) j(w) \sim \frac{\boldsymbol{\varepsilon}}{(z-w)^{2}},
\end{gathered}
$$

whose charge operator $j_{0}$ counts $\boldsymbol{c}=+1, \boldsymbol{b}=-1$ charge. The conformal properties of the current depend on the vacuum chosen. In the $\mathrm{SL}_{2}$ invariant vacuum sector $\langle\boldsymbol{c}(z) \boldsymbol{b}(w)\rangle=1 /(z-w)$ and there are no finite corrections to the singularity (26); then the proper relation is

$$
\begin{aligned}
T(z) j(w) & \sim \frac{Q}{(z-w)^{3}}+\frac{j(z)}{(z-w)^{2}}, \\
{\left[L_{m}, j_{n}\right] } & =-n j_{m+n}+\frac{1}{2} Q m(m+1) \delta_{m,-n},
\end{aligned}
$$

so that $j(z)$ is scale and translation covariant $(m=0,-1)$ but not conformally covariant $(m=+1)$. Rather, we find

$$
\begin{gathered}
{\left[L_{1}, j_{-1}\right]=j_{0}+Q,} \\
{\left[L_{1}, j_{-1}\right]^{\dagger}=\left[L_{-1}, j_{1}\right]=-j_{0},}
\end{gathered}
$$

which shows the charge asymmetry of the system: $j_{0}^{\dagger}=-\left(j_{0}+Q\right)$. Consequently operator expectation values of charge neutral operators will vanish since

$$
\begin{aligned}
{\left[j_{0}, O\right] } & =q O, \\
\left\langle 0\left|j_{0} O\right| 0\right\rangle & =-Q\langle 0|O| 0\rangle=q\langle 0|O| 0\rangle .
\end{aligned}
$$

Only operators which cancel the background charge $Q$ survive; this feature was encountered in our discussion of the reparametrization ghosts (cf. eq. (37)) where we 
had to introduce ghost operators in amplitudes to soak up the three zero modes $(Q=-3)$ of $c$. In general there is an index (cf. eq. (35))

$$
\text { \# zero modes of } \boldsymbol{c}-\# \text { zero modes of } \boldsymbol{b}=-\frac{1}{2} \varepsilon Q \chi \text {, }
$$

where $\chi=2(1-g)$ for a surface with $g$ handles.

The number current may be used to build a stress energy tensor [61] that reproduces the relation (76):

$$
\begin{aligned}
T_{j}(z) & =\varepsilon\left(-\frac{1}{2} j(z)^{2}-\frac{1}{2} Q \partial_{z} j_{z}\right), \\
T_{j}(z) j(w) & \frac{Q}{(z-w)^{3}}+\frac{j(z)}{(z-w)^{2}}, \\
T_{j}(z) j(w) & \sim \frac{\frac{1}{2}\left(1-3 \varepsilon Q^{2}\right)}{(z-w)^{4}} .
\end{aligned}
$$

Thus the new stress tensor has $c_{j}=1-3 \varepsilon Q^{2}$; the original $b, c$ stress tensor had

$$
\begin{array}{lll}
c=c_{j}, & \varepsilon=+1 & \text { (Fermi), } \\
c=c_{j}-2, & \varepsilon=-1 & \text { (Bose) },
\end{array}
$$

Since in the first case there is no "leftover" stress-energy, the current $j$ and its stress tensor $T_{j}$ completely characterize the Fermi theory. This is why, for instance, the spacetime fields $\psi^{\mu}$ can be described solely on the basis of $\operatorname{SO}(9,1)$ current algebra. For Bose systems, however, there is a residual $\boldsymbol{c}=-2$ stress tensor $T_{-2}$ which commutes with $j$ and $T_{j}$, such that

$$
T=T_{j}+T_{-2}
$$

From our formula (75) we see that such a system may be represented in terms of an auxiliary linear Fermi system with $\lambda_{-2}=1, Q_{-2}=-1$, composed of a dimension-1 field $\eta(z)$ and a dimension- 0 field $\xi(z)$. Using this auxiliary system and the current $j$ we can rewrite the Bose $\boldsymbol{b}, \boldsymbol{c}$ field theory.

As in the case of group current algebra, it is convenient to bosonize $j$ by defining

$$
\begin{gathered}
j(z)=\varepsilon \partial_{z} \phi, \\
\phi(z) \phi(w) \sim \varepsilon \ln (z-w) .
\end{gathered}
$$


The exponentials of the line integral of the current $\mathrm{e}^{q \phi(z)}$ transform as

$$
\begin{aligned}
& T(z) \mathrm{e}^{q \phi(w)} \sim\left[\frac{\frac{1}{2} \varepsilon q(q+Q)}{(z-w)^{2}}+\frac{1}{z-w} \partial_{w}\right] \mathrm{e}^{q \phi(w)}, \\
& j(z) \mathrm{e}^{q \phi(w)} \sim \frac{q}{z-w} \mathrm{e}^{q \phi(w)}, \\
& {\left[j_{0}, \mathrm{e}^{q \phi(w)}\right]=q \mathrm{e}^{q \phi(w)},}
\end{aligned}
$$

so $\mathrm{e}^{q \phi}$ shifts the charge by $q$ and has conformal weight $\frac{1}{2} \varepsilon q(q+Q)$. The action

$$
S_{Q}=\frac{1}{4 \pi} \int \mathrm{d}^{2} z\left[2(-\varepsilon) \partial_{z} \phi \partial_{\bar{z}} \phi-\frac{1}{2} Q \sqrt{g} R \phi\right]
$$

describes the bosonized current; indeed the equation of motion

$$
\partial_{z} \partial_{\bar{z}} \phi=\frac{1}{8} \varepsilon Q \sqrt{g} R
$$

precisely reproduces the current anomaly (76), (34). In terms of our new variable(s) $\phi$ (and $\eta, \xi$ for Bose systems) we may write $b$ and $c$ as

$$
\begin{array}{lll}
\boldsymbol{c}(z)=\mathrm{e}^{\phi(z)}, & \boldsymbol{b}(z)=\mathrm{e}^{-\phi(z)} & \text { (Fermi), } \\
\boldsymbol{c}(z)=\mathrm{e}^{\phi(z)} \eta(z), & \boldsymbol{b}(z)=\mathrm{e}^{-\phi(z)} \partial \xi(z) & \text { (Bose), }
\end{array}
$$

The solitons $\mathrm{e}^{ \pm \phi}$ are always fermions, thus $\eta, \xi$ must be used to make $\boldsymbol{b}$ and $c$ in the Bose case. A useful exercise is to verify (74) by inserting the representative (79) into the 1.h.s. and using OPE's to generate the r.h.s. This gives a complete description of the $\boldsymbol{b}, \boldsymbol{c}$ field theory; in particular this bosonized form conveniently represents the spin fields $\Sigma=\mathrm{e}^{ \pm \phi / 2}$ which interpolate between the NS and R sectors (cf. (60)). It is curious and essential for future developments that the zero mode $\xi_{0}$ never appears in the $\boldsymbol{b}, \boldsymbol{c}$ algebra of Bose systems; only derivatives of $\xi$ are needed. This means the irreducible representation of $\boldsymbol{b}$ and $c$ is built out of $\phi, \eta$, and $\rho=\partial \xi$; inclusion of $\xi_{0}$ makes the representation reducible. Then every state in the system $|\phi\rangle$ would have a degenerate partner $\xi_{0}|\phi\rangle$ with exactly the same properties under the current $j(z)$. We must discuss one final point before returning to strings. In some of the foregoing analysis we assumed that the vacuum $|0\rangle$ was $\mathrm{SL}_{2}$ invariant. However the spectrum is unbounded above and below, so the choice of vacuum state is somewhat arbitrary. This point is familiar in the case of Fermi statistics, where a Fermi sea-level must be specified; likewise, for Bose statistics we must state the energy level below which all the levels are filled. If we were describing an interacting field theory, there could be transitions between levels, leading to a collapse of the vacuum; but 
since we are dealing with a free field theory, this prescription causes no catastrophe. We will call this the Bose sea-level. Therefore we define the different vacua $|q\rangle$ by

$$
\begin{aligned}
& b_{n}|q\rangle=0, \quad n>\varepsilon q-\lambda, \\
& c_{n}|q\rangle=0, \quad n \geqslant-\varepsilon q+\lambda,
\end{aligned}
$$

where for the NS sector $q \in \frac{1}{2}+\mathbb{Z}$ and in the $\mathrm{R}$ sector $q \in \mathbb{Z}$. The adjoint of the $q$-vacuum is a state $\langle-q-Q|$ such that $\langle-q-Q \mid q\rangle=1$. The propagator is no longer a simple pole in this vacuum; it receives finite corrections from the vacuum charge:

$$
\begin{aligned}
\langle\boldsymbol{c}(z) \boldsymbol{b}(w)\rangle_{\mathrm{q}} & =\sum_{m, n}\left\langle-q-Q\left|\boldsymbol{c}_{m} \boldsymbol{b}_{n}\right| q\right\rangle z^{-m-(1-\lambda)} w^{-n-\lambda} \\
& =\sum_{n \leqslant \varepsilon-q-\lambda}\left\langle q-Q\left|\left[\boldsymbol{c}_{-n}, \boldsymbol{b}_{n}\right]_{\varepsilon}\right| q\right\rangle z^{n-(1-\lambda)} w^{-\eta-\lambda}, \\
\langle\boldsymbol{c}(z) \boldsymbol{b}(w)\rangle_{\mathrm{q}} & =\left(\frac{z}{w}\right)^{\varepsilon q} \frac{1}{z-w} .
\end{aligned}
$$

The conformal properties of the current and stress-energy tensor are modified as a result

$$
\begin{aligned}
\langle T(z) j(w)\rangle & \sim \frac{Q}{(z-w)^{3}}+\frac{1}{(z-w)^{2}} \frac{q}{z}, \\
j(w) & =: j(w):+\frac{q}{w}, \\
\langle T(z) T(w)\rangle & \sim \frac{\frac{1}{2} c}{(z-w)^{4}}+\frac{\varepsilon q(q+Q)}{(z-w)^{2}} \frac{1}{z w}, \\
T(z) & =: T(z):+\frac{1}{2} \varepsilon q(q+Q) \frac{1}{z^{2}} .
\end{aligned}
$$

These relations give the charge and energy of the $q$-vacuum as

$$
\begin{aligned}
j_{0}|q\rangle & =q|q\rangle \\
L_{0}|q\rangle & =\varepsilon q(q+Q)|q\rangle
\end{aligned}
$$

and it is a simple exercise to check that $L_{-1}$ annihilates only the state $|0\rangle$, the $\mathrm{SL}_{2}$ invariant vacuum. A crucial distinction between the Bose and Fermi cases is that for Fermi systems $(\varepsilon=+1)$ the sea-level is shifted by the action of $\boldsymbol{b}$ and $\boldsymbol{c}$ whereas for Bose systems the $q$-vacua generate inequivalent representations of the $\boldsymbol{b}, \boldsymbol{c}$ algebra - a 
finite number of field operators cannot fill a state. However, from (78) we see that the coherent state operators $: \mathrm{e}^{q \phi}$ : interpolate between the various Bose sea-levels, shifting the vacuum charge by $q$ since

$$
|q\rangle=\mathrm{e}^{q \phi(0)}|0\rangle
$$

From this we may derive (80) via the operator product expansion of $\mathrm{e}^{q \phi}$ with (79); then expanding $b(z), c(z)$ in modes we see that the terms singular as $z \rightarrow 0$ must multiply annihilation operators. A final note: the auxiliary $\xi, \eta$ system has two degenerate vacua $|0\rangle, \xi|0\rangle$, such that $\langle 0|\xi| 0\rangle=1$. Upon reducing to the irreducible $\boldsymbol{b}, \boldsymbol{c}$ representation in terms of $\eta, \partial \xi=\rho$, the auxiliary field vacuum is unique: $\langle 0 \mid 0\rangle=1$ since the $\xi$ zero mode is absent.

\subsection{THE COVARIANT FERMION VERTEX}

Having acquired all the technology of linear field theories, let us explore the applications in string theory. Our original reason for launching into the above discourse was to find the spin operators for the spinor ghosts $\beta, \gamma$. Through bosonizing the ghost number current in the manner of current algebras, we obtained a scalar field $\phi$ whose exponentials $\mathrm{e}^{q \phi}$ of dimension $-\frac{1}{2} q(q+2)$ shift the spinor ghost vacuum charge by $q$. In particular, the exponentials $\mathrm{e}^{\phi / 2}$ of dimension $\frac{3}{8}$ and $\mathrm{e}^{-\phi / 2}$ of dimension $-\frac{5}{8}$ alter the vacuum by a half unit of charge and interpolate between the NS and R ground states, and are therefore potential spin fields $\Sigma$. Our first candidate covariant fermion vertex is

$$
\begin{aligned}
V_{-1 / 2}(u, k, z) & =u^{\alpha}(k) \mathrm{e}^{-\phi(z) / 2} S_{\alpha}(z) \mathrm{e}^{i k \cdot X(z)}, \\
k u & =k^{2}=0 .
\end{aligned}
$$

It is straightforward to verify that the operator products of $V_{-1 / 2}$ with both $j_{\mathrm{BRST}}^{(1)}$ and $j_{\mathrm{BRST}}^{(2)}$ are nonsingular and hence $\int V_{-1 / 2}$ commutes with $Q_{\mathrm{BRST}}=Q_{0}+Q_{1}+Q_{2}$. This is, however, not sufficient to describe fermion scattering because $V_{-1 / 2}$ has spinor ghost charge of $-\frac{1}{2}$, and only the four-point amplitude would cancel the spinor ghost background charge $Q=2$. Thus we need a second version of the fermion vertex, $V_{1 / 2}$, with opposite spinor ghost charge. The obvious candidate ghost operator is $\mathrm{e}^{\phi / 2}$ of dimension $-\frac{5}{8}$, however, its operator product with $j_{\mathrm{BRST}}^{(1)}$ is more singular than $\mathrm{e}^{-\phi / 2}$. In fact, the only operators which commute with $Q_{\text {BRST }}$ with positive ghost charge are of the form $\left[Q_{\mathrm{BRST}}, O\right]$. These are all spurious, with the exception of

$$
V_{\text {phys }}^{\prime}=\left[Q_{\mathrm{BRST}}, \xi V_{\mathrm{phys}}\right]
$$

which is not spurious because $\xi$ is not part of the irreducible algebra of the spinor ghosts $\beta$ and $\gamma$. Recall that our irreducible current algebra representation involves $\phi$, 
$\eta$, and $\rho=\partial \xi$ but not $\xi$ itself. Hence $\xi V_{\text {phys }}$ is not in the irreducible vertex operator algebra, and consequently $\left[Q_{\mathrm{BRST}}, \xi V_{\text {phys }}\right]$ is not spurious or null in this algebra. In fact, the inclusion of the $\xi$ zero-mode renders the vacuum non-invariant under the BRST algebra. To see this, let us examine the $\mathrm{SL}_{2}$ invariant vacuum $|0\rangle$, for which

$$
\begin{array}{ll}
b_{n}|0\rangle=0, & n \geqslant-1, \\
c_{n}|0\rangle=0, & n \geqslant 2, \\
\beta_{n}|0\rangle=0, & n \geqslant-\frac{1}{2}, \\
\gamma_{n}|0\rangle=0, & n \geqslant \frac{3}{2} .
\end{array}
$$

It follows from these properties that

$$
Q_{\mathrm{BRST}}|0\rangle=0 .
$$

To calculate, it is convenient to bosonize all the ghosts, including the auxiliary $\xi, \eta$ system:

$$
\begin{gathered}
c=\mathrm{e}^{\sigma}, \quad b=\mathrm{e}^{-\sigma}, \\
\gamma=\mathrm{e}^{\phi} \eta=\mathrm{e}^{\phi-\chi}, \quad \beta=\mathrm{e}^{-\phi} \partial \xi=\mathrm{e}^{-\phi} \rho=\mathrm{e}^{-\phi+\chi} \partial \chi, \\
\langle\chi(z) \chi(w)\rangle=\langle\sigma(z) \sigma(w)\rangle=-\langle\phi(z) \phi(w)\rangle=\ln (z-w) .
\end{gathered}
$$

In the $\eta, \rho$ algebra, the conjugate of the state $|0\rangle$ is $\mathrm{e}^{3 \sigma-2 \phi}(0)|0\rangle$, and

$$
Q_{\text {BRST }} \mathrm{e}^{3 \sigma-2 \phi}(0)|0\rangle=0 .
$$

Hence vacuum expectation values of physical vertex operators

$$
A_{N} \sim\left\langle 0\left|\mathrm{e}^{3 \sigma-2 \phi} V_{\text {phys }}(1) \ldots V_{\text {phys }}(N)\right| 0\right\rangle
$$

will be BRST-invariant. On the other hand, in the $\eta, \xi$ algebra the conjugate of $|0\rangle$ is $\mathrm{e}^{3 \sigma-2 \phi+\chi}(0)|0\rangle$ because of the $\xi$ zero-mode; one readily verifies that

$$
Q_{\mathrm{BRST}} \mathrm{e}^{3 \sigma-2 \phi+\chi}(0)|0\rangle \neq 0,
$$

so that vacuum expectation values in the reducible representation of the ghost algebra are not manifestly BRST-invariant. Nevertheless it is often convenient to pass back and forth between these two algebras by including or removing the $\xi$ zero mode integration in the path integral. For instance, although $V_{\text {phys }}^{\prime}$ in (83) exists in both algebras, its representation in intermediate steps of a calculation as a BRSTcommutator in the reducible algebra is quite useful, as we shall see. 
Returning to our search for fermion vertex operators, we find that

$$
\begin{aligned}
V_{1 / 2}(u, k, z) & =\left[Q_{\mathrm{BRST}}, 2 \xi V_{-1 / 2}\right] \\
& =\left[Q_{1}+Q_{2}, 2 \xi V_{-1 / 2}\right] \\
& =u^{\alpha}(k)\left\{\mathrm{e}^{\phi / 2}\left[\partial X^{\mu}+\frac{1}{4} i(k \cdot \psi) \psi^{\mu}\right] \gamma_{\mu \alpha \beta} S^{\beta}+\frac{1}{2} \mathrm{e}^{3 \phi / 2} \eta b S_{\alpha}\right\} \mathrm{e}^{i k \cdot X}
\end{aligned}
$$

is the physical vertex operator of spinor ghost charge opposite to $V_{-1 / 2}$. We have ignored a total derivative which will not contribute when the vertex is integrated over the world sheet. The fact that the product of $V_{-1 / 2}$ and $V_{1 / 2}$ factorizes on the NS vector vertex (71) demonstrates that this is indeed the correct procedure. This product is most easily evaluated by writing $V_{1 / 2}$ as a BRST-commutator as in eq. (85)

$$
\begin{aligned}
V_{-1 / 2}(u, k, z) V_{1 / 2}(v, p, w) & =V_{-1 / 2}(z)\left[Q_{1}, 2 \xi V_{-1 / 2}(w)\right] \\
& =-2\left[Q_{1}, V_{-1 / 2}(z) \xi V_{-1 / 2}(w)\right]
\end{aligned}
$$

We can ignore the second piece of $V_{1 / 2}$ because it cannot contribute to expectation values involving $V_{-1 / 2}$ due to $b$-charge conservation. The product

$$
V_{-1 / 2}(u, k, z) V_{-1 / 2}(v, p, w) \sim(z-w)^{-1+k \cdot p}\left(\bar{u} \gamma^{\mu} v\right) \mathrm{e}^{-\phi} \psi^{\mu} \mathrm{e}^{i(p+k) \cdot X}(w)
$$

is now substituted on the right-hand side and evaluated as

$$
\begin{aligned}
V_{-1 / 2}(u, k, z) V_{1 / 2}(v, p, w) \\
-(z-w)^{-1+p \cdot k}\left(\bar{u} \gamma^{\mu} v\right) \oint \frac{\mathrm{d} z^{\prime}}{2 \pi i} \mathrm{e}^{\phi} \eta \psi \cdot \partial X\left(z^{\prime}\right) \mathrm{e}^{-\phi} \xi \psi^{\mu} \mathrm{e}^{i(p+k) \cdot X}(w) \\
\left.-(z-w)^{-1+p \cdot k}\left(\bar{u} \gamma^{\mu} v\right)\left[\partial X^{\mu}+i(p+k) \cdot \psi \psi^{\mu}\right) \mathrm{e}^{i(p+k) \cdot X}\right] \\
\sim(z-w)^{-1+p \cdot k} V_{\mathrm{B}}\left(\xi^{\mu}=\bar{u} \gamma^{\mu} v, p+k, w\right) .
\end{aligned}
$$

Note again that, though we worked in the enlarged $\eta, \xi$ algebra in intermediate steps, the end result does not involve $\xi$ and is consequently valid in the $\eta, \rho$ subalgebra as well. The desired fermion vertex thus has (so far) two versions

$$
V_{\mathrm{F}}(u, k, z)=V_{-1 / 2} \quad \text { or } \quad V_{1 / 2} .
$$

We should mention that, although $S^{\beta}$ in $V_{1 / 2}$ has the opposite chirality compared to $S_{\beta}$ in $V_{-1 / 2}, V_{1 / 2}$ carries one more unit of spinor ghost charge which contributes to 
make the overall value of $(-)^{\mathrm{F}}$ identical. To calculate a fermion scattering process we just put together charge neutral combinations of $V_{-1 / 2}$ and $V_{1 / 2}$.

It is fortunate that operators of the form (83) are not spurious, else no sensible fermion vertex would exist. Instead we now have an embarassment of riches, for not only does $V_{-1 / 2} V_{1 / 2}$ factorize on physical NS vertices, but so do $V_{-1 / 2} V_{-1 / 2}$ and $V_{1 / 2} V_{1 / 2}$ ! For instance, (86) is a second physical NS vector vertex, in addition to (71). Any expectation value with the vertices occurring in the (time) order

$$
\left\langle\ldots V_{-1 / 2} V_{1 / 2} V_{-1 / 2} V_{1 / 2} \ldots\right\rangle
$$

will necessarily contain the unwanted orderings in crossed channels, by duality. These operator products factorize the amplitude on different Bose sea-levels (cf. eq. (81)) than that of the canonical $\mathrm{SL}_{2}$ invariant vacuum because of the nonzero $\phi$-charge of the operators. All is well if we can show that all these different sea levels are equivalent. To do so, consider any correlation function of fermion vertices $V_{\mathrm{F}}$. It is a correlation function containing an equal number of $V_{-1 / 2}$ and $V_{1 / 2}$ vertices by $\phi$-charge conservation. If we can prove that

$$
\begin{aligned}
& \left\langle\ldots V_{-1 / 2}\left(u_{1}, k_{1}, z_{1}\right) \ldots V_{1 / 2}\left(u_{2}, k_{2}, z_{2}\right) \ldots\right\rangle \\
& \quad=\left\langle\ldots V_{1 / 2}\left(u_{1}, k_{1}, z_{1}\right) \ldots V_{-1 / 2}\left(u_{2}, k_{2}, z_{2}\right) \ldots\right\rangle,
\end{aligned}
$$

then by a succession of such operations we can arrange the vertices in the canonical order (88); intermediate states of different Bose sea levels are equivalent descriptions with the same physical content, and we can always choose to work in the canonical NS and R sectors.

The proof of (89) involves passing back and forth between the "big" $(\eta, \xi)$ and "small" $(\eta, \rho)$ algebras in the functional integral, as in the steps leading to the demonstration (87) of factorization. Begin with two vertices $V_{-1 / 2}$ at $z_{1}$ and $V_{1 / 2}$ at $z_{2}$ in a correlation function in the small algebra (see fig. 2a). Now move to the large algebra; this means merely that we include in the path integral an integration over the $\xi$ zero-mode, together with a factor $\xi(z)$ in the amplitude to absorb the Grassmann integration. So far we have done nothing. Note that the location of the extra $\xi(z)$ on the world sheet is irrelevant since only the constant part contributes; therefore, choose $z=z_{1}$. In the large algebra we may now write $V_{1 / 2}=$ $\left[Q_{\mathrm{BRST}}, \xi V_{-1 / 2}\right]$ in terms of the contour integral of the current $j_{\mathrm{BRST}}$ about $z_{2}$ (see fig. $2 b)$ :

$$
V_{1 / 2}\left(z_{2}\right)=\oint \frac{\mathrm{d} w}{2 \pi i} j_{\mathrm{BRST}}(w) \xi\left(z_{2}\right) V_{-1 / 2}\left(z_{2}\right)
$$

Recall that in the large algebra, $Q_{\mathrm{BRST}} \xi|0\rangle \neq 0$ and so the calculation is no longer manifestly BRST invariant; otherwise eq. (85) would imply that $V_{1 / 2}$ decouples, being a BRST commutator, and all fermion amplitudes would vanish. 


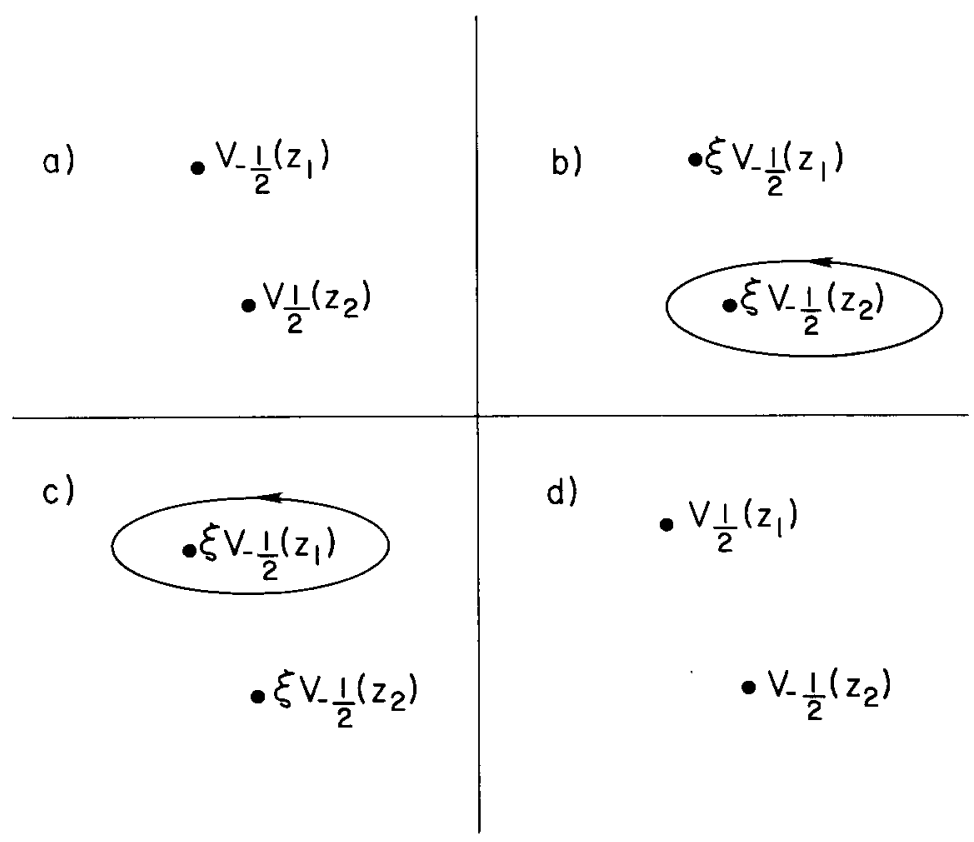

Fig. 2. Steps leading to the demonstration of Bose sea equivalence.

Now we may deform the contour by pulling it off the back of the sphere. In general there will be other physical vertices in the correlation function but because $Q_{\text {BRST }}$ (anti)commutes with them the contour integral passes through without generating new terms. The contour now surrounds $z_{1}$ instead of $z_{2}$ (see fig. 2c); the orientation is as shown due to the Fermi statistics of the Grassmann $\xi$ integral. Using eq. (85) we see that we have successfully interchanged the two types of vertices, and integrating out the $\xi$ zero-mode we transfer back to the small algebra (fig. 2 d). Thus the identity (89) is proved. Since $\xi$ has dimension 0 , there is always a single constant zero mode of $\xi$ for any world-sheet topology, and the preceding argument generalizes. The $j_{\mathrm{BRST}}$ contour integral passes through handles by splitting and rejoining. Zero modes of $b, c$, and the other ghosts do not vitiate the argument; as explained in sect. 2, the reparametrization ghost zero-mode insertions do not affect BRST contour deformations, and similar considerations show no difficulties with the spinor ghosts either (see sect. 7). World surfaces which have boundaries or are nonorientable can be treated similarly; one should find a problem with BRST invariance unless the gauge group is $\mathrm{SO}(32)$.

This $\xi$-manipulation exposes a remarkable redundancy in the representation of the fermion scattering amplitudes. Because the vertices $V_{-1 / 2}$ and $V_{1 / 2}$ carry spinor ghost charge, intermediate states factorize on differing Bose sea-levels of the $\beta, \gamma$ system; thus the Hilbert space must be extended to include all the inequivalent 
representations of this algebra corresponding to the different vacuum sectors. Nevertheless, any physical state has a representative in each sector connected to all its relatives by the $\xi$-manipulation, so all the Bose sea-levels are equivalent under the algebra generated by the vertex operators. The fermion vertex generates a closed subalgebra of this hugely overextended matter-ghost system.

The $\xi$-manipulation that generates $V_{1 / 2}$ from $V_{-1 / 2}$ may also be used to generate further vertices (which we label by their Bose sea charges)

$$
\begin{aligned}
& V_{3 / 2}=\left[Q_{\mathrm{BRST}}, \xi V_{1 / 2}\right], \\
& V_{5 / 2}=\left[Q_{\mathrm{BRST}}, \xi V_{3 / 2}\right],
\end{aligned}
$$

and so on. Similarly, we may find the vertex $V_{-3 / 2}$ such that $V_{-1 / 2}=$ $\left[Q_{\mathrm{BRST}}, \xi V_{-3 / 2}\right] \cdot \mathrm{e}^{-3 \phi / 2} S^{\alpha} \mathrm{e}^{i k \cdot X}$ is the BRST invariant vertex with Bose sea charge - $\frac{3}{2}$ which produces the highest weight NS vector vertex in its operator product with $V_{1 / 2}$ and has $V_{-1 / 2}$ as its successor under a Bose sea shift. Proceeding in this way we may produce a version of any vertex operator that interpolates between any two Bose sea-levels of opposite grading (NS and R). Similarly, there is also an infinite tower of NS vertices, of which $V_{-1}$ is the "canonical" highest weight vector vertex, and the superfield $V_{0}=V_{\mathrm{B}}$ (eq. (87)) is its successor. The rearrangement lemma implies that we need only choose one representative charge-neutral collection of vertices in correlation functions since any other configuration is equivalent. Preliminary investigation indicates that the $\xi$-manipulation is invertible in the space of physical states, although we have no general proof.

In fact, a glimmer of this redundancy was discovered in the early days of the NS theory. From (84) we see that the $\mathrm{SL}_{2}$ invariant vacuum is not annihilated by either of the ghost modes $c_{1}$ or $\gamma_{1 / 2}$; not all the positive frequency mode operators propagate forward in time. In other words, although $|0\rangle$ is a highest weight state of the super-Virasoro algebra, it is not a highest weight state of the ghost algebra. For the reparametrization ghosts, we saw in sect. 2 that the situation could be remedied by changing the Fermi sea-level of the $b, c$ system.

$$
c(0)|0\rangle=\mathrm{e}^{\sigma(0)}|0\rangle
$$

is the highest weight vacuum state of energy -1 , corresponding to the conventional tachyon ground state of the bosonic string. Similarly, the highest weight vacuum of the combined ghost system is

$$
c(0) \mathrm{e}^{-\phi(0)}|0\rangle
$$

This state has energy $-\frac{1}{2}$ and fermion parity $\Gamma=-1$; it is the canonical NS 
tachyon ground state. Converting between this Bose sea level and $|0\rangle$ can be accomplished via the $\xi$-manipulation and was known in past times as "changing pictures" [62]; the procedure used then was to write the initial and final vertices of a scattering process as commutators with $G_{ \pm 1 / 2}$ :

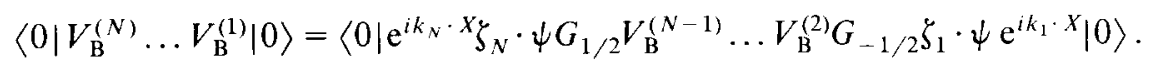

Then moving $G_{-1 / 2}$ off to the left, one generates a new representation of the amplitude where the ground state has energy $-\frac{1}{2}$ instead of -1 . We may now understand this rather obscure operation as a $\xi$-manipulation; by writing

$$
V_{\mathrm{B}}(\zeta, k, z)=\left[Q_{\text {BRST }}, \xi \mathrm{e}^{-\phi} \zeta(k) \cdot \psi \mathrm{e}^{i k \cdot x}(x)\right],
$$

we have (recall that $\left\langle 0\left|\mathrm{e}^{-2 \phi+3 \sigma}\right| 0\right\rangle=1$ )

$$
\begin{aligned}
\left\langle 0\left|\mathrm{e}^{-2 \phi+3 \sigma} V_{\mathrm{B}}^{(N)} \ldots V_{\mathrm{B}}^{(1)}\right| 0\right\rangle & \\
= & \left\langle 0\left|\mathrm{e}^{-2 \phi+3 \sigma} \xi V_{\mathrm{B}}^{(N)} \ldots V_{\mathrm{B}}^{(2)}\left[Q_{\mathrm{BRST}}, \xi \mathrm{e}^{-\phi} \zeta_{1} \cdot \psi \mathrm{e}^{i k_{1} \cdot X}\right]\right| 0\right\rangle \\
& =\left\langle 0\left|\mathrm{e}^{-\phi+3 \sigma} \xi_{N} \cdot \psi \mathrm{e}^{i k_{N} \cdot X} V_{\mathrm{B}}^{(N-1)} \ldots V_{\mathrm{B}}^{(2)} \mathrm{e}^{-\phi} \xi_{1} \cdot \psi \times \mathrm{e}^{i k_{1} \cdot X}\right| 0\right\rangle
\end{aligned}
$$

and we we that the "picture changing" operation of the old formalism has a very natural interpretation as a rewriting of the theory in a different ghost vacuum!

\subsection{SAMPLE CALCULATIONS}

To illustrate the use of all these formal procedures in a practical calculation, consider the example of two-fermion-two-boson scattering (see fig. 3a). The matrix element to be calculated is

$$
\begin{aligned}
G_{2 \mathrm{~F} 2 \mathrm{~B}}(1 \ldots 4)= & \left\langle\mathrm{e}^{-2 \phi+3 \sigma \zeta_{4}} \cdot\left(\partial X+i k_{4} \cdot \psi \psi\right) \mathrm{e}^{i k_{4} \cdot X}\left(z_{4}\right) \zeta_{3} \cdot\left(\partial X+i k_{3} \cdot \psi \psi\right) \mathrm{e}^{i k_{3} \cdot X}\left(z_{3}\right)\right. \\
& \left.\times u_{2}^{\alpha} S_{\alpha} \mathrm{e}^{-\phi / 2} \mathrm{e}^{i k_{2} \cdot X}\left(z_{2}\right) u_{1}^{\beta} \gamma_{\beta \gamma}^{\mu} S^{\gamma}\left[\partial X^{\mu}+\frac{1}{4} i k_{1} \cdot \psi \psi^{\mu}\right] \mathrm{e}^{\phi / 2} \mathrm{e}^{i k_{1} \cdot X}\left(z_{1}\right)\right\rangle .
\end{aligned}
$$

Only the parts of the fermion vertex $V_{1 / 2}$ which contribute to the correlation function have been written - we consistently drop terms which don't conserve ghost charge. A $\xi$-manipulation simplifies the calculation - go to the "large" algebra and move both $\xi(z)$ and $\mathrm{e}^{-2 \phi(z)}$ to $z_{4}$. Now write $V_{1 / 2}=\left[Q_{\mathrm{BRST}}, \xi V_{-1 / 2}\right]$ as a BRST contour integral and deform the contour onto $\xi \mathrm{e}^{2 \phi} V_{\mathrm{B}}\left(z_{4}\right)$. The relevant operator product gives

$$
\begin{aligned}
& \eta \mathrm{e}^{\phi} \partial X \cdot \psi(w) \xi \mathrm{e}^{-2 \phi \xi_{4}} \cdot\left(\partial X+i k_{4} \cdot \psi \psi\right) \mathrm{e}^{i k_{4} \cdot X}\left(z_{4}\right) \\
& \quad \sim(z-w)^{1+2-2} \mathrm{e}^{-\phi} \zeta_{4} \cdot \psi \mathrm{e}^{i k_{4} \cdot X}\left(z_{4}\right)+\left(\text { terms } \propto k^{2} \text { or } \zeta \cdot k\right)+\text { finite } .
\end{aligned}
$$


a)

$$
\begin{gathered}
x^{e^{3 \sigma-2 \phi}} \\
v_{-\frac{1}{2}\left(z_{2}\right)} \\
\dot{V}_{0}\left(z_{4}\right) \quad \dot{V}_{0}\left(z_{3}\right)^{V_{\frac{1}{2}}\left(z_{1}\right)}
\end{gathered}
$$

b)

$$
\cdot c V-\frac{1}{2}(1)
$$

$\stackrel{\bullet}{c V_{-1}}(\infty) \bullet V_{0}\left(z_{3}\right)$

Fig. 3. Vertex configurations for the two-fermion-two-boson scattering amplitude (a) original configuration, and (b) after a Bose sea shift.

Thus $V_{\mathrm{B}}\left(z_{4}\right)$ is converted into a "picture-changed" NS vertex (fig. 3b). In addition we can fix the location of vertices 1,2 , and 4 as in sect. 2 by attaching $c=\mathrm{e}^{\sigma}$ to them and dropping the Koba-Nielsen integration. We have

$$
\begin{aligned}
G_{2 \mathrm{~F} 2 \mathrm{~B}}=\left\langle\zeta_{4} \cdot\right. & \psi \mathrm{e}^{-\phi+\sigma} \mathrm{e}^{i k_{4} \cdot X}\left(z_{4}\right) \zeta_{3} \cdot\left(\partial X+i k_{3} \cdot \psi \psi\right) \mathrm{e}^{i k_{3} \cdot X}\left(z_{3}\right) \\
& \left.\times u_{2}^{\alpha} S_{\alpha} \mathrm{e}^{-\phi / 2+\sigma} \mathrm{e}^{i k_{2} \cdot X}\left(z_{2}\right) u_{1}^{\beta} S_{\beta} \mathrm{e}^{-\phi / 2+\sigma} \mathrm{e}^{i k_{1} \cdot X}\left(z_{1}\right)\right\rangle .
\end{aligned}
$$

Now evaluate each of the component field correlations. The ghost contributions are trivial:

$$
\begin{gathered}
\left\langle\mathrm{e}^{\sigma\left(z_{4}\right)} \mathrm{e}^{\sigma\left(z_{2}\right)} \mathrm{e}^{\sigma\left(z_{1}\right)}\right\rangle=\left(z_{4}-z_{2}\right)\left(z_{2}-z_{1}\right)\left(z_{1}-z_{4}\right), \\
\left\langle\mathrm{e}^{-\phi\left(z_{4}\right)} \mathrm{e}^{-\phi / 2\left(z_{2}\right)} \mathrm{e}^{-\phi / 2\left(z_{1}\right)}\right\rangle=\left(z_{4}-z_{2}\right)^{-1 / 2}\left(z_{4}-z_{1}\right)^{-1 / 2}\left(z_{2}-z_{1}\right)^{1 / 4} .
\end{gathered}
$$

The two possible contributions of $X^{\mu}$ are also easy:

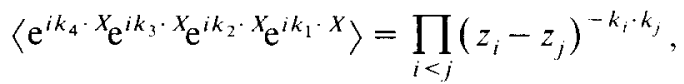

$$
\begin{aligned}
& \left\langle\mathrm{e}^{i k_{4} \cdot X \zeta_{3}} \cdot \partial X \mathrm{e}^{i k_{3} \cdot X} \mathrm{e}^{i k_{2} \cdot X^{i k_{1} \cdot X}}\right\rangle=\left[\prod_{i<j}\left(z_{i}-z_{j}\right)^{-k_{i} \cdot k_{j}}\right] \\
& \times i\left(\frac{\zeta_{3} \cdot k_{4}}{z_{3}-z_{4}}+\frac{\zeta_{3} \cdot k_{2}}{z_{3}-z_{2}}+\frac{\zeta_{3} \cdot k_{1}}{z_{3}-z_{1}}\right) .
\end{aligned}
$$

At first sight the calculation of the fermion correlations might appear formidable, but this is not the case. They are determined solely by their $\operatorname{SO}(9,1)$ and conformal 
properties. We require that the correlation functions transform in the appropriate representation with the correct conformal weight at each point. For instance, this uniquely specifies

$$
\left\langle\psi^{\mu}\left(z_{4}\right) S_{\alpha}\left(z_{2}\right) S_{\beta}\left(z_{1}\right)\right\rangle=\left(z_{4}-z_{2}\right)^{-1 / 2}\left(z_{4}-z_{1}\right)^{-1 / 2}\left(z_{2}-z_{1}\right)^{-3 / 4} \gamma_{\alpha \beta}^{\mu},
$$

such that the singularities in each pair $\left(z_{i}-z_{j}\right)$ match the operator products (59) [13]. The other correlation function we shall need is just the insertion of the Lorentz current $j^{\nu \lambda}=\psi^{\nu} \psi^{\lambda}$ into the above; its effect is to produce rotations of the fields [35] (cf. eq. (58))

$$
\begin{aligned}
& \left\langle j^{\nu \lambda}\left(z_{3}\right) \psi^{\mu}\left(z_{4}\right) S_{\alpha}\left(z_{2}\right) S_{\beta}\left(z_{1}\right)\right\rangle \\
& \quad=\sum_{i \neq 3} \frac{M_{i}^{\nu \lambda}}{z_{3}-z_{i}}\left\langle\psi^{\mu}\left(z_{4}\right) S_{\alpha}\left(z_{2}\right) S_{\beta}\left(z_{1}\right)\right\rangle \\
& \quad=\sum_{i \neq 3} \frac{M_{i}^{v \lambda}}{z_{3}-z_{i}} \gamma_{\alpha \beta}^{\mu}\left(z_{4}-z_{2}\right)^{-1 / 2}\left(z_{4}-z_{1}\right)^{-1 / 2}\left(z_{2}-z_{1}\right)^{-3 / 4},
\end{aligned}
$$

where $M_{i}^{\mu \nu}$ is the Lorentz generator acting on the index at $z_{i}$. Combining (90)-(91), we find

$$
\begin{aligned}
G_{2 \mathrm{~F} 2 \mathrm{~B}}(1 \ldots 4)=\prod_{i<j}\left(z_{i}-z_{j}\right)^{-k_{i} \cdot k_{j}} \times\{ & {\left[\frac{\zeta_{3} \cdot k_{4}}{z_{3}-z_{4}}+\frac{\zeta_{3} \cdot k_{2}}{z_{3}-z_{2}}+\frac{\zeta_{3} \cdot k_{1}}{z_{3}-z_{1}}\right] u_{2} \zeta_{4} u_{1} } \\
& +\frac{1}{z_{3}-z_{4}}\left(\zeta_{4} \cdot k_{3} u-2 \xi_{1}-\zeta_{4} \cdot \zeta_{3} u_{2} k_{3} u_{1}\right) \\
& +\frac{\frac{1}{2}}{z_{3}-z_{2}}\left(\zeta_{4}^{\mu} k_{3}^{v} \zeta_{3}^{\lambda} u_{2} \gamma^{[v} \gamma^{\lambda]} \gamma^{\mu} u_{1}\right) \\
& \left.+\frac{\frac{1}{2}}{z_{3}-z_{1}}\left(\zeta_{4}^{\mu} k_{3}^{v} \zeta_{3}^{\lambda} u_{2} \gamma^{\mu} \gamma^{[v} \gamma^{\lambda]} u_{1}\right)\right\} .
\end{aligned}
$$

To produce the full amplitude, we must combine this result with an appropriate correlation function in $\bar{z}$ and integrate $z_{3}, \bar{z}_{3}$ over the complex plane. For instance, in the heterotic string the $\bar{z}$ correlations are those of the bosonic string (19). The quantum numbers of Yang-Mills particles are carried by left-moving currents $j_{\bar{z}}^{a}$ or other conformal fields of $\operatorname{Spin}(32) / Z_{2}$ or $\mathrm{E}_{8} \times \mathrm{E}_{8}$ current algebra; alternatively (or in addition) the $\bar{z}$-dependent part of the vertex may carry Lorentz indices. The 
relevant correlation for a four-point gauge particle amplitude is

$$
\begin{aligned}
& \bar{G}_{2 \mathrm{~F} 2 \mathrm{~B}}(1 \ldots 4) \\
& =\left\langle\mathrm{e}^{\bar{\sigma}} \mathrm{e}^{i k_{4} X} j_{a_{4}}\left(\bar{z}_{4}\right) \mathrm{e}^{i k_{3} X} j_{a_{3}}\left(\bar{z}_{3}\right) \mathrm{e}^{\bar{\sigma}} \mathrm{e}^{i k_{2} X} j_{a_{2}}\left(\bar{z}_{2}\right) \mathrm{e}^{\bar{\sigma}} \mathrm{e}^{i k_{1} X} j_{a_{1}}\left(\bar{z}_{1}\right)\right\rangle \\
& =\prod_{i<j}\left(\bar{z}_{j}-\bar{z}_{i}\right)^{k_{1} k_{j}}\left\{\left[\left(\bar{z}_{1}-\bar{z}_{2}\right)\left(\bar{z}_{1}-\bar{z}_{4}\right)\left(\bar{z}_{3}-\bar{z}_{2}\right)\left(\bar{z}_{3}-\bar{z}_{4}\right)\right]^{-1} \operatorname{tr}\left(T_{a_{1}} T_{a_{2}} T_{a_{3}} T_{a_{4}}\right)\right. \\
& +\left[\left(\bar{z}_{1}-\bar{z}_{2}\right)\left(\bar{z}_{1}-\bar{z}_{3}\right)\left(\bar{z}_{4}-\bar{z}_{2}\right)\left(\bar{z}_{4}-\bar{z}_{3}\right)\right]^{-1} \operatorname{tr}\left(T_{a_{1}} T_{a_{2}} T_{a_{4}} T_{a_{3}}\right) \\
& +\left[\left(\bar{z}_{1}-\bar{z}_{3}\right)\left(\bar{z}_{1}-\bar{z}_{4}\right)\left(\bar{z}_{2}-\bar{z}_{3}\right)\left(\bar{z}_{2}-\bar{z}_{4}\right)\right]^{-1} \operatorname{tr}\left(T_{a_{1}} T_{a_{3}} T_{a_{2}} T_{a_{4}}\right) \\
& +\left(\bar{z}_{1}-\bar{z}_{2}\right)^{-2}\left(\bar{z}_{3}-\bar{z}_{4}\right)^{-2} \operatorname{tr}\left(T_{a_{1}} T_{a_{2}}\right) \operatorname{tr}\left(T_{a_{3}} T_{a_{4}}\right) \\
& +\left(\bar{z}_{1}-\bar{z}_{3}\right)^{-2}\left(\bar{z}_{2}-\bar{z}_{4}\right)^{-2} \operatorname{tr}\left(T_{a_{1}} T_{a_{3}}\right) \operatorname{tr}\left(T_{a_{2}} T_{a_{4}}\right) \\
& \left.+\left(\bar{z}_{1}-\bar{z}_{4}\right)^{-2}\left(\bar{z}_{2}-\bar{z}_{3}\right)^{-2} \operatorname{tr}\left(T_{a_{1}} T_{a_{4}}\right) \operatorname{tr}\left(T_{a_{2}} T_{a_{3}}\right)\right\} \\
& \times\left(\bar{z}_{4}-\bar{z}_{2}\right)\left(\bar{z}_{2}-\bar{z}_{1}\right)\left(\bar{z}_{1}-\bar{z}_{4}\right) .
\end{aligned}
$$

The integral of $G \cdot \bar{G}$ over $z_{3}, \bar{z}_{3}$ is then the desired four-string $s$-matrix element. The integration yields a combination of $\Gamma$-functions, kinematic factors and group traces:

$$
\begin{aligned}
A_{\mathrm{tot}}= & \pi\left(u_{2} \gamma \cdot \zeta_{4} \gamma \cdot\left(k_{3}+k_{1}\right) \gamma \cdot \zeta_{3} u_{1}\right)\left[\operatorname{tr}(1234) \frac{2}{s}+\operatorname{tr}(1243) \frac{2 t}{s u}+\operatorname{tr}(1324) \frac{2}{u}\right. \\
& \left.+\operatorname{tr}(12) \operatorname{tr}(34) \frac{-2 t}{s(s+2)}+\operatorname{tr}(13) \operatorname{tr}(24) \frac{-2 t}{u(u+2)}+\operatorname{tr}(14) \operatorname{tr}(23) \frac{2}{t}\right] \\
+ & \pi\left(u_{2} \gamma \cdot \zeta_{3} \gamma \cdot\left(k_{3}+k_{2}\right) \gamma \cdot \zeta_{4} u_{1}\right)\left[\operatorname{tr}(1234) \frac{2}{t}+\operatorname{tr}(1243) \frac{2}{u}+\operatorname{tr}(1324) \frac{2 s}{t u}\right. \\
& \left.+\operatorname{tr}(12) \operatorname{tr}(34) \frac{2}{s}+\operatorname{tr}(13) \operatorname{tr}(24) \frac{-2 s}{u(u+2)}+\operatorname{tr}(14) \operatorname{tr}(23) \frac{-2 s}{t(t+2)}\right] \\
& \times \frac{\Gamma\left(-\frac{1}{2} u\right) \Gamma\left(-\frac{1}{2} t\right) \Gamma\left(-\frac{1}{2} s\right)}{\Gamma\left(\frac{1}{2} u\right) \Gamma\left(\frac{1}{2} t\right) \Gamma\left(\frac{1}{2} s\right)} .
\end{aligned}
$$

The ratio of three $\Gamma$-functions approaches one in the limit $\alpha^{\prime} \rightarrow 0$; away from this it reveals the poles associated with massive string state resonances. The explicit poles 
appearing with the group traces (which are taken in the adjoint representation with an implicit factor of $\frac{1}{30}$ ) represent the massless particle poles, which receive contributions from all states carrying the relevant quantum numbers.

Conformal techniques may of course also be used to calculate multifermion scattering processes, although the algebra is more involved. In particular, the four-fermion amplitude [52a] has been recomputed [53]. Starting with the configuration

$$
G_{4 \mathrm{~F}}=\left\langle V_{-1 / 2} V_{1 / 2} V_{-1 / 2} V_{1 / 2}\right\rangle,
$$

one can again use a $\xi$-manipulation to turn all four vertices into $V_{-1 / 2}$ 's. The ghost correlation function

$$
\left\langle\mathrm{e}^{-\phi(x) / 2} \mathrm{e}^{-\phi(1) / 2} \mathrm{e}^{-\phi(z) / 2} \mathrm{e}^{-\phi(0) / 2}\right\rangle=[z(1-z)]^{-1 / 4}
$$

reproduces in a simple way (using only free field theory) the part of the scattering amplitude that required a computer to discover in the previous formulation of the fermion vertex [56]. The four- $S_{\alpha}$ correlation can be worked out by a variety of methods, with the answer [53]

$$
\left\langle S_{\alpha}(\infty) S_{\beta}(1) S_{\gamma}(z) S_{\delta}(0)\right\rangle=[z(1-z)]^{-3 / 4}\left((1-z) \gamma_{\alpha \beta}^{\mu} \gamma_{\gamma \delta}^{\mu}-z \gamma_{\alpha \delta}^{\mu} \gamma_{\beta \gamma}^{\mu}\right)
$$

The computation of the amplitude using the appropriate $\bar{z}$ correlation function proceeds as before.

If one is willing to forego the manifest symmetries of the $\operatorname{SO}(9,1)$ and group currents, the calculations are simplified by working in the bosonic representation described in sect. 4 (see also refs. $[57,12]$ ); for instance, $V_{-1 / 2}$ becomes

$$
V_{1}(u, k, z)=u(k) \mathrm{e}^{-\phi / 2} \mathrm{e}^{i \alpha \cdot H^{i k \cdot X}} .
$$

The Lorentz algebra is somewhat ugly due to the explicit choice of basis for the Dirac algebra and the grouping of Lorentz indices in pairs, but this is more than compensated by the fact that all the correlations are just those of free fields! The bosonized form is also convenient for the analysis of multiloop diagrams, where the construction of the free field Neumann function on a general two-dimensional surface is a classical problem solved in the last century. We will give an outline of this analysis in sect. 7.

\section{Supersymmetry}

We have given a prescription for the determination of all the operators of the fermionic string theory using BRST invariance. In particular the vertices for massless states have been exhibited, and a few sample correlation functions calculated. In this 
section, we will investigate the supersymmetry of the fermionic string theory in the covariant quantization. We will construct the covariant supersymmetry charge, thus proving supersymmetry to all orders in string perturbation theory (modulo anomalies), and show that supersymmetry guarantees the vanishing of tadpole diagrams and mass shifts of massless particles. We conjecture a relationship between the NSR and other approaches to covariant quantization of fermionic strings [23,24].

The supersymmetry charge should be a BRST invariant operator which interchanges the NS-boson and R-fermion sectors of the string Hilbert space, relating states of the same mass. The natural candidate is the line integral of the fermion vertex at zero momentum

$$
Q_{\alpha}=\oint \frac{\mathrm{d} z}{2 \pi i} V_{\alpha}^{\mathrm{F}} \quad(k=0)
$$

From the operator product relation (87), we find that $Q_{\alpha}$ converts a massless fermion emission vertex into a massless boson emission vertex:

$$
\left[Q_{\alpha}, V_{\mathrm{F}}(u, k, z)\right]=V_{\mathrm{B}}\left(\zeta^{\mu}=u^{\beta} \gamma_{\beta \alpha}^{\mu}, k, z\right)
$$

The converse is readily demonstrated as well:

$$
\left[Q_{\alpha}, V_{\mathrm{B}}(\zeta, k, z)\right]=V_{\mathrm{F}}\left(u^{\beta}=i k^{\mu} \zeta^{\nu}\left(\gamma_{\mu \nu}\right)_{\alpha}^{\beta}, k, z\right)
$$

The BRST invariance of $V_{\alpha}^{\mathrm{F}}$ guarantees that of $Q_{\alpha}$. Generally we may write any fermion (boson) vertex as the contour integral of $V_{\alpha}^{\mathrm{F}}$ about its boson (fermion) vertex partner, i.e. as a commutator with $Q_{\alpha}$ (see fig. 4). On a multiply-handled Riemann surface, this demonstrates the vanishing of tadpole diagrams. Upon writing the vertex as a supersymmetry commutator, the analytic contour integral may be pulled off the back of the surface; since in the process no singularities are encountered, the diagram vanishes. This argument is specific to the theory projected onto even chirality $\Gamma=1$. Before this projection there are square root cuts in correlation functions and phase ambiguities in transporting an operator around a handle. Upon projecting onto $\Gamma=1$ we have a local field theory on the world sheet and no global phase ambiguities in the remaining operators (this property is largely

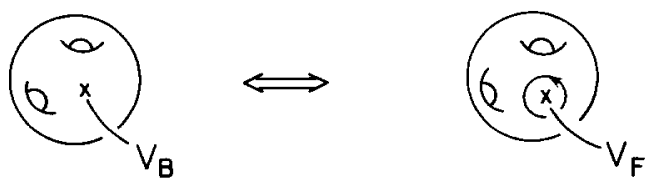

Fig. 4. Rewriting a boson vertex insertion as a supersymmetry commutator. 


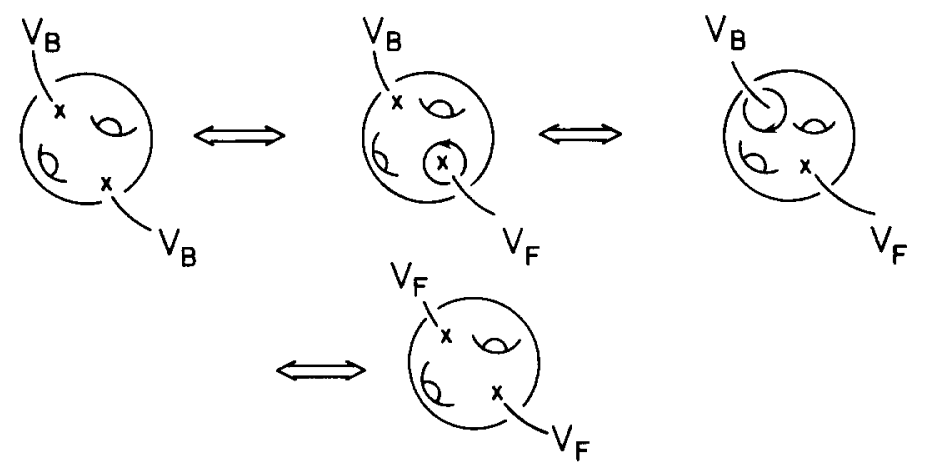

Fig. 5. Supersymmetry contour deformation relating boson and fermion two-point functions.

a result of modular invariance). The same contour manipulation on a two-point function relates boson and fermion propagator corrections since this time the deformed supersymmetry contour circles the second vertex (see fig. 5). Chirality thereby protects against mass renormalizations of massless bosons.

The vector and spinor vertices at zero momentum

$$
\begin{aligned}
& P^{\mu}(z) \sim \partial_{z} X^{\mu}, \\
& Q_{\alpha}(z) \sim \mathrm{e}^{-\phi / 2} S_{\alpha}(z) \quad \text { or } \quad \mathrm{e}^{\phi / 2} S^{\beta} \gamma_{\beta \alpha}^{\mu} \partial_{z} X^{\mu}
\end{aligned}
$$

(and their images in other pictures) are like a supersymmetry "current algebra"; more precisely their line integrals are a set of conserved charges obeying

$$
\begin{aligned}
& {\left[P^{\mu}, P^{\nu}\right]=\left[P^{\mu}, \boldsymbol{Q}_{\alpha}\right]=0,} \\
& {\left[\boldsymbol{Q}_{\alpha}, \boldsymbol{Q}_{\beta}\right]=2 \gamma_{\alpha \beta}^{\mu} P^{\mu} .}
\end{aligned}
$$

The currents themselves don't quite form a closed algebra, since for example

$$
P^{\mu}(z) Q_{\alpha}(w) \sim \frac{1}{(z-w)^{2}} \mathrm{e}^{\phi / 2} S^{\beta} \gamma_{\beta \alpha}^{\mu}(w)
$$

Because of relations like (94), the current would-be current algebra does not close on a finite number of Bose sea-levels. To obtain a closed algebra would require working in all the different pictures simultaneously; this may be required for string field theory. Nevertheless the form (93) is rather suggestive. One would like to interpret $\mathrm{e}^{\phi / 2} S^{\alpha}(z)$ as $\theta^{\alpha}(z)$, the dimension-zero supersymmetry partner of $X^{\mu}$, with $\mathrm{e}^{\phi / 2} S_{\alpha}$ its charge conjugate $\delta / \delta \theta^{\alpha}(z)$. Eqs. (93)-(94) look vaguely like Siegel's 
relations [24]

$$
\begin{aligned}
& P^{\mu}(z) P^{\nu}(w) \sim \frac{1}{(z-w)^{2}}, \\
& D_{\alpha}(z) D_{\beta}(w) \sim \frac{1}{z-w} \gamma_{\alpha \beta}^{\mu} P^{\mu}(z) \quad\left(D_{\alpha} \sim \frac{\delta}{\delta \theta^{\alpha}}-\theta P_{\alpha}\right), \\
& P^{\mu}(z) D_{\alpha}(w) \sim \frac{1}{z-w} \partial_{z} \theta^{\beta} \gamma_{\beta \alpha}^{\mu}
\end{aligned}
$$

and one may show that the natural candiate for a $\theta^{\alpha}$ stress-energy tensor

$$
\partial_{z} \theta^{\alpha} \frac{\delta}{\delta \theta^{\alpha}}=16\left[\frac{1}{2}\left(\partial_{z} \psi^{\mu}\right) \psi^{\mu}\right]+4\left[\frac{1}{2}\left(\partial_{z} \phi\right)^{2}+\partial_{z}^{2} \phi\right]
$$

is a linear combination of the Lorentz and ghost current stress-energy tensors. If one could find an appropriate interpretation of $\theta^{\alpha}$, perhaps a lagrangian could be written which quantizes the superstring variables directly much as Green and Schwarz have done on the light cone [63]. One would then have a $2 \mathrm{~d}$ field theory which directly quantizes the spin model, and supersymmetry would be manifest. A geometrical classical action has been written for $X^{\mu}$ and $\theta^{\alpha}$ [23], but only recently has the full algebra of first-class constraints been discovered [24]. If this program succeeds, one will have another quantization of superstrings in terms of $X^{\mu}, \theta^{\alpha}$, and the Faddeev-Popov ghosts for fixing the local symmetries of the action. This should not yield a new string theory, rather we expect that there will exist at the quantum level a change of variables relating the two (BRST quantized) theories in much the same way that a triality rotation in $S O(8)$ relates the light-cone NSR and GreenSchwarz theories [64]. The covariant $\theta^{\alpha}$ should look something like our candiate $\theta^{\alpha}$; the Faddeev-Popov ghost for the local fermionic symmetries [23,24] of the GreenSchwarz-Siegel (GSS) action (generated by the dimension-2 "current" $P D$ ) is a dimension -1 field, probably $\eta \mathrm{e}^{3 \phi / 2} S_{\alpha} \equiv \kappa$. Then the part of the GSS BRST operator $\kappa \not P D$, which serves to restrict to the physical half of the components of $\theta^{\alpha}$ in the GSS theory, would reproduce $\gamma P \cdot \psi$ in the NSR theory which also restricts to physical fermion polarizations. Proceeding in this way one should be able to make a complete correspondence between the string variables plus ghosts of the two quantizations. The symmetry algebra of the GSS action is rather complicated [24], so we see that the NSR variables provide an extremely compact embodiment of the on-shell physics. It is unclear whether the supersymmetry charge (92) will generate full off-shell supersymmetry. $Q_{\alpha}$ shifts the Bose sea-charge by some amount; to get a closed off-shell algebra, one would have to work in a Hilbert space which is the direct sum of all the different Bose sea Hilbert spaces since the Bose sea equivalence given by the $\xi$-manipulation is only valid on-shell. It is possible that all the auxiliary fields needed for off-shell supersymmetry in ten dimensions could arise from the various vertex operators in different Bose seas, but it is not clear how. Since the 
first-quantized formalism is gauge-fixed, presumably one would be working in some particular (covariant) supersymmetry gauge [65].

\section{String perturbation theory}

The study of string loop perturbation theory is just in its infancy. Loop "graphs" correspond to path integrals over Riemann surfaces $\mathfrak{S}$ with some number of handles and can be thought of as being obtained by "sewing together" tree diagrams [66] with a sum over states in the Hilbert space of the sewn external legs. In practice, this procedure boils down to a brute force construction of the path integral on a higher-genus Riemann surface, we will begin by describing the construction of such surfaces (including the moduli) and then give a prescription for evaluating the path integral. In the process we will encounter some elegant classical mathematics, and conformal technology will prove its worth once again [67].

In the mathematical literature, a Riemann surface $S$ is usually described in terms of its universal covering space $\tilde{\delta}$, such that $\mathcal{S}$ is $\tilde{\delta}$ modulo some infinite discrete subgroup $\Gamma$ of $\operatorname{SL}(2, \mathrm{R})$ which acts on $\tilde{\delta}$ without fixed points; $\Gamma$ is in fact $\Pi_{1}(\delta)$. These parametrizations of $\delta$ have an extensive mathematical literature [68] and are closely tied to the metric of the surface and differential geometry. Another approach uses a different parametrization of the surface. Investigations during the previous incarnation of string theory produced this parametrization [45] in the course of treating loop diagrams as the unitarization of the tree-level $S$-matrix. The group $\operatorname{SL}(2, C)$ maps the plane $\hat{C}$ onto itself. An element $\gamma \in \operatorname{SL}(2, C)$

$$
\gamma(z)=\frac{a z+b}{c z+d}, \quad a d-b c=1
$$

( $a, b, c, d$ complex) may be rewritten

$$
\frac{\gamma(z)-\xi}{\gamma(z)-\eta}=K\left(\frac{z-\xi}{z-\eta}\right)
$$

in terms of the fixed points $\xi, \eta=(1 / 2 c)\left(a-d \pm \sqrt{(a+d)^{2}-4}\right)$ and the multiplier $K+K^{-1}=a+d-2$. Without loss of generality we may choose $|K|<1$, so that $\eta$ is repulsive and $\xi$ is attractive. The rate of traversal from one to the other governed by $K . \gamma$ maps the circle $\mathcal{C}=\{z:|c z+d|=1\}$ into the circle $\mathcal{C}^{\prime}=\{z:|c z-a|=1\}$, known as the isometric circles of $\gamma$. The identification of $\hat{\mathrm{C}}$ under $\gamma$ generates a torus, i.e., if we cut holes in the sphere along $C$ and $\mathcal{C}^{\prime}$ and glue the boundaries together we build a handle (see fig. 6). For the torus, $\gamma$ may be chosen to be a dilatation $\gamma(z)=\omega z$; it is sometimes convenient to use the parametrization $\tilde{z}=$ $(\ln z) / 2 \pi i$ (and thus $\tau=(\ln \omega) / 2 \pi i)$. Surfaces of higher-genus $g>1$ are obtained by considering the group $\Gamma$ generated by $g$ elements $\gamma_{i} \in \operatorname{SL}(2, \mathrm{C}), i=1, \ldots, g$. The $\gamma_{i}$ must be such that no isometric circle separates any two other isometric circles - then the group would not generate a surface. Since $\gamma_{i}$ maps $\mathcal{C}_{i}$ into $\mathcal{C}_{i}^{\prime}$, the 


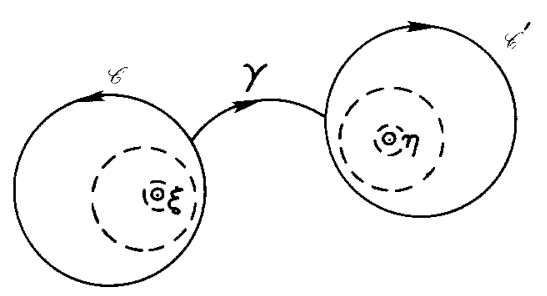

Fig. 6. Identification of two circles in the complex plane under an SL(2,C) transformation $\gamma$ generates a handle. The dotted circles are the images of $c$ under powers of $\gamma \cdot \xi$ and $\eta$ are the fixed points of the transformation.

region exterior to all the $\mathcal{C}_{i}, e_{i}^{\prime}(i=1 \ldots g)$ is a fundamental domain such that any $\gamma \in \Gamma$ maps a point inside this region to a point outside, and so identification under the action of $\Gamma$ yields the $g$-torus. $\Gamma$ is known as a Schottky group [69]. The circles $e_{i}, e_{i}^{\prime}$ joined by $\gamma_{i}$ correspond to a cutting of the surface $\delta$ along the $g$ cycles $c_{2 i-1}$ of a canonical homology basis of $S$ (see fig. 7). The generating elements $\gamma_{i}$ comprise $6 \mathrm{~g}$ parameters, of which 6 may be fixed by an overall conjugation by an element of $\operatorname{SL}(2, \mathrm{C})$, leaving $6 g-6$ free parameters as moduli of the surface $\delta$. Note that such a conjugation cannot alter the trace of a matrix, hence the single complex modulus remaining in the torus case.

Given the surface $\mathfrak{S}$ in terms of the group $\Gamma$, one of the important tasks is to construct a basis for holomorphic one-forms, $f(z) \mathrm{d} z$. Such a basis is given by the so-called abelian differentials of the first kind $\mathrm{d} \omega_{i}, i=1 \ldots g$ [45]. These act like holomorphic coordinate differentials for transport around a given handle. The integrals of these holomorphic one-forms are the first abelian integrals and are defined by their periodicity properties along the cycles generating $H_{1}(\delta)$, the first homology group. One may always choose a canonical set of cycles $c_{1} \ldots c_{2 g}$ as depicted in fig. 7. The basis $\mathrm{d} \omega_{i}$ is then determined by the "period matrix" of intersections

$$
\begin{gathered}
\oint_{c_{2 i-1}} \mathrm{~d} \omega_{j}=2 \pi i \cdot \delta_{i j} \\
\oint_{c_{2 i}} \mathrm{~d} \omega_{j}=2 \pi i \cdot \pi_{i j}
\end{gathered}
$$

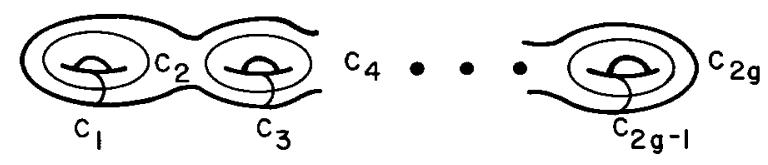

Fig. 7. Standard homology basis for the surface. 
The first equation is a set of normalization conditions. The matrix $\pi_{i j}$ and the $\mathrm{d} \omega_{i}$ are related to the group $\Gamma$ as we shall see in a moment. Indeed one may take any $3 g-3$ of the $\frac{1}{2} g(g+1)$ complex entries of $\pi_{i j}$ as a set of local moduli, but they are less directly related to construction of the surface and the Neumann function, and no fixed set of $3 g-3$ periods are valid global coordinates on moduli space. The exception is the torus, where $\mathrm{d} \omega=\mathrm{d} z$, the standard coordinate differential; and the relations (95) give the distances 1 and $\tau$ around the two noncontractable loops. The Poincaré theta series

$$
\theta(z, a) \mathrm{d} z=\sum_{\gamma \in \Gamma} \frac{\mathrm{d} \gamma(z)}{\gamma(z)-a}=\sum_{\gamma \in \Gamma} \frac{\mathrm{d} z}{\left(c_{\gamma} z+d_{\gamma}\right)^{2}} \frac{1}{\gamma(z)-a}
$$

is an automorphic form (i.e., a function covariant with respect to the group $\Gamma$ ) such that

$$
\mathrm{d} \omega_{i}=\theta(z, a) \mathrm{d} z-\theta\left(z, \gamma_{i}(a)\right) \mathrm{d} z
$$

are our set of first abelian differentials. That is, $\theta(z, a)$ is holomorphic except for the pole at $z=a$; the difference (96) subtracts the pole to leave a holomorphic function which is covariant under $\Gamma$. Moreover, the so-called "third abelian differential"

$$
\mathrm{d} \omega_{a b}=\theta(z, a)-\theta(z, b),
$$

is such that its integral

$$
\omega_{a b}(z)-\omega_{a b}\left(z_{0}\right)=-\sum_{\gamma \in \Gamma} \log \left[\frac{\gamma(z)-a}{\gamma(z)-b} \frac{\gamma\left(z_{0}\right)-b}{\gamma\left(z_{0}\right)-a}\right]
$$

has the correct singularity structure to be the Green function on the surface $\delta$. The poles of $\mathrm{d} \omega_{a b}$ in other fundamental regions are the image charges used to build up the correct Green function. The addition of a linear combination of first abelian integrals $\omega_{i}(z)-\omega_{i}\left(z_{0}\right)=\int_{z_{0}}^{z} \mathrm{~d} \omega_{i}$ will not change the singularity structure but does alter the integral of a function around a cycle; the appropriate definition of the Green function turns out to be

$$
\begin{aligned}
V\left(z_{m}, z_{n} ; a, b\right)= & \operatorname{Re}\left[\omega_{z_{n} b}\left(z_{m}\right)-\omega_{z_{n} b}(a)\right] \\
& +\sum_{i=1}^{g}\left[\operatorname{Re}\left(\omega_{i}\left(z_{m}\right)-\omega_{i}(a)\right)\right](\operatorname{Im} \pi)_{i j}^{-1}\left[\operatorname{Re}\left(\omega_{j}\left(z_{n}\right)-\omega_{j}(b)\right)\right]
\end{aligned}
$$


The extra piece is chosen so that $V$ is single-valued on the surface

$$
\oint_{\mathcal{C}_{i}} \mathrm{~d} V=0, \quad i=1, \ldots, 2 g
$$

Since we have seen that all of the operators of the fermionic and bosonic string theories have realizations in terms of free scalar fields, this is sufficient to evaluate all correlation functions. One works in the bosonic representation of the Lorentz current algebra $j^{\mu \nu}$ (and group currents $j^{a}$ for heterotic strings) and also of the ghost fields $b, c, \beta, \gamma$. The oscillator modes of the bosons $X^{\mu}, H_{i}, \sigma, \phi$, and $\chi$ generate the third abelian integral contribution to the two-point function $V$; the zero-mode loop integrations (or lattice sums as the case may be) yield the holomorphic first abelian integral terms [45]. The two-cocycles associated with each of the lattices should keep track of minus signs in spacetime fermion loops.

One of the difficulties of previous attempts to construct multiloop amplitudes was an ambiguity as to the measure of integration. The vertex operator contribution to the amplitude is simply given by the set of free field contractions using the two-point function $V$; the measure is more subtle. First, one needs to construct the vacuum energy contribution to the diagram. As we saw in sect. 2, the ghosts enter in an essential way in order to cancel the propagation of spurious states, a mechanism not available in the days of dual models. The second derivative $\left.\partial_{z} \partial_{w} V(z, w)\right|_{z \rightarrow w}$ gives the derivative of the vacuum energy in terms of the stress tensor of all the bosons as in sect. 2. Given the variation of the metric in terms of our parametrization of the surface, we can integrate with respect to the moduli as in (36) to obtain the partition function. In the case of the fermionic string, these factors conspire to cancel at one-loop due to fermion zero modes [70]. For fermionic string multiloop diagrams, spacetime fermion and boson contributions to the partition function to continue to cancel in the vacuum energy [67]. Another approach to the calculation of the relevant functional determinants is pursued in ref. [45], where loop diagrams are calculated by sewing tree diagrams as mentioned above. The missing component in this work was the ghost partition function. One obtains the determinants of the relevant laplacians in terms of the multipliers of the group $\Gamma$ :

$$
\operatorname{det} \Delta^{\prime}=\prod_{\gamma}^{\prime} \prod_{n=1}^{\infty}\left(1-K_{\gamma}^{n}\right)^{2} \text {. }
$$

The prime on the 1.h.s indicates the omission of the zero-mode contribution, and on the r.h.s denotes a product over elements which are not powers of other elements. In the case of $X^{\mu}$ the zero modes just contribute factors of $\operatorname{det}\left(\operatorname{Im} \pi_{i j}\right)$ to the measure; for the other (bosonized) fields the zero-mode momenta lie on a lattice and there will be finite corrections.

In addition to the dependence of the vacuum partition function on the moduli, which we discussed in sect. 2 , in the fermionic string there are "supermoduli" [60]. 
The Riemann-Roch theorem (77) tells us there are $2(g-1)$ complex gravitino zero modes for $g>1$ (also: on the sphere $G_{ \pm 1 / 2}, \bar{G}_{ \pm 1 / 2}$ act as the four conformal Killing spinors, and on the torus there are two supermoduli and two conformal Killing spinors $G_{0}, \bar{G}_{0}$, depending on the spin structure). Of course, for the heterotic string we keep only the holomorphic part of this superstructure. The supermoduli are the modes of the gravitino field which cannot be gauged away in choosing the superconformal gauge (50). Integration over the supermoduli will bring factors of $T_{\mathrm{F}}(z, \theta)$ down from the action (49), folded in with the appropriate zero-mode wave functions. The ghosts will also reflect these zero modes. In the bosonized representation, there will be a background charge coupled to the bosonized current $j=\partial \phi$. The counting of the background charge is the same as for the gravitino zero modes. BRST invariance of the measure provides a unique prescription for incorporating the effects of the supermoduli and the background $\phi$-charge. The combination

$$
\left[Q_{\mathrm{BRST}}, \xi\right]_{+}=-\frac{1}{2} \mathrm{e}^{\phi} P \cdot \psi-\frac{1}{4} \partial \eta \mathrm{e}^{2 \phi} b-\frac{1}{4} \partial\left(\eta \mathrm{e}^{2 \phi} b\right)+c \partial \xi
$$

joins the background charge operator $\mathrm{e}^{\phi}$ of the spinor ghosts with the $T_{\mathrm{F}}$ produced by the gravitino zero modes (both have the same counting - two for each handle) in a BRST invariant way. Note that the combination has $(-)^{\mathrm{F}}=1$ and is therefore a good spin model operator; the separate components have odd fermion parity. The $\eta$ zero modes (cf. (77)) may be treated by the method used for $b$. BRST contour integrals pass through all these extra zero modes painlessly.

The volume element and domain of integration of the moduli are determined by invariance under the group of global diffeomorphisms mod( $\delta$ ) (the modular group), and also by the requirement that longitudinal modes decouple by the BRST contour deformation argument. In the case of the torus $\bmod (S)$ is given by

$$
\hat{\Gamma}(\tau)=\frac{a \tau+b}{c \tau+d}, \quad a, b, c, d \in \mathbb{Z} ; \quad a d-b c=1
$$

The integration measure invariant under this group is $\mathrm{d}^{2} \tau /(\operatorname{Im} \tau)^{2}$ and a fundamental domain is shown in fig. 8. The universal covering space $T(\delta)$ of the moduli space (in this case the upper half-plane $\boldsymbol{H}$ ) contains the dangerous point $\tau=0$; the two-point function $V$ has an essential singularity as $\tau \rightarrow 0$ because all the image charges on $\tilde{\delta}$ pile up at the same point. The transformation $\hat{\gamma}(\tau)=-1 / \tau$ sends this "ultraviolet" limit to the "infrared" point $\tau=i \infty$ (think of Im $\tau$ as the proper distance around the time direction of the torus). It is the restriction to the fundamental region $R(\delta)=T(\delta) / \bmod (\delta)$ of fig. 8 , allowed by the absence of global diffeomorphism anomalies $[47,70,12,52]$, which guarantees the one-loop finiteness of fermionic strings $[70,12]$ - the singularity is outside the integration region (other domains which have $\tau=0$ as a limit point have vanishing measure there). For higher-genus surfaces, the technical proof of ultraviolet finiteness works 


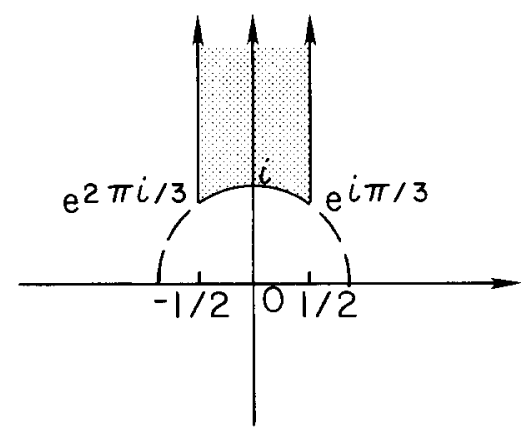

Fig. 8. Fundamental domain for the modulus of the torus.

the same way. ${ }^{\star}$ Infrared divergences associated with massless particles are absent due to supersymmetry, at least for the one- and two-point functions, due to the contour argument of sect. 6 . Hence it seems that string perturbation theory should be finite - there are no ultraviolet divergences by modular invariance, and no infrared singularities by supersymmetry.

\section{Background fields}

We have seen that the conformal invariance of strings propagating in flat Minkowski space plays an important role in the structure of the theory. One of the reasons to explore conformal field theory is that this structure generalizes in a straightforward manner to a theory of strings propagating in nontrivial backgrounds $[25,26,27]$. In a nontrivial background, the tree-level string theory is an interacting $2 \mathrm{~d}$ quantum field theory. Although the stress tensor is still (anti)analytic, conformal fields no longer factorize into products of analytic and antianalytic fields. Our arguments about BRST invariance, supersymmetry, etc. have been tailored not to depend on the special features of flat space string theory. Rather, they require only that the relevant charge is the line integral of a world sheet current, i.e. a dimension $(1,0)$ or $(0,1)$ operator. Eventually one must understand whatever nonlinear invariance underlies string theory. Conformal invariance is a kind of criterion for splitting the string between on-shell classical background and quantum fluctuation. It is a remarkable fact that, in contrast to first-quantized particle theories, demanding conformal invariance in first-quantized string theories provides the equations de-

* Physically, one expects all along that string theories are ultraviolet finite. In the days of hadronic dual models, one difficulty was that the form factor for deep inelastic scattering decreased like a gaussian instead of a power law because there was nothing "hard" inside a string to scatter off of. Now we regard this as desirable; strings are floppy on the scale $\sqrt{\alpha^{\prime}}$, and there are no short distance divergences because there is nothing small enough in the theory to probe those distance scales. Of course, infrared problems associated with tachyons and dilatons [71] are another story; see [67]. 
termining the environment in which they live - a necessary criterion for the string to yield a unified theory. The language of conformal field theory allows us to express various features of string compactification and "phenomenology" in terms of properties of scale invariant two-dimensional field theory. In particular, this approach may be useful for studying the properties of the two-dimensional theory regardless of whether the string fluctuations are strongly coupled to the background; by studying the conformal algebra and its consequences, one may even be able to find strong coupling solutions if enough inputs can be found.

For the moment, however, let us consider the bosonic string in a weakly coupled background. Physically this means that the typical size of a string, $\sqrt{\alpha^{\prime}}$, is small compared to the radius of the manifold, so the "spin-wave" approximation is valid. Interactions with the background are introduced as in first-quantized particle theory; there, in the path representation of a particle propagator [72]

$$
\Delta\left(x, x^{\prime}\right)=\int \mathscr{D} x \operatorname{QD} \exp \left[i \int_{0}^{1} \sqrt{g} \dot{x}^{2} \mathrm{~d} \tau\right]
$$

an interaction with a background gauge field is introduced through a coupling $\int A \cdot \dot{x}$ in the action, and a background spacetime metric changes the inner product contracting the $x^{\mu}$ in (99). The choice of gauge $g=$ const is the analogue of the conformal gauge; it also leaves a residual invariance under constant shifts in $\tau$ generated by the particle hamiltonian

$$
H=\left(p^{\mu}-A^{\mu}\right) G_{\mu \nu}\left(p^{\nu}-A^{\nu}\right) .
$$

This symmetry must be imposed as a constraint on the particle Hilbert space after quantization

$$
H\left|\Phi_{\text {phys }}\right\rangle=0
$$

Since the configuration space of classical field theory is just the first-quantized Hilbert space, eq. (100) is just the equation of motion $\nabla^{2} \Phi(x)=0$.

The sum over surfaces in the two-dimensional nonlinear sigma model gives a first-quantized string propagator in a curved background. Reparametrization invariance is again the key to consistency. String has the additional property that it contains gravity, so the equations of motion derived from reparametrization invariance must also determine the background in which string fluctuations propagate. The first-quantized description of particles is not suitable for the description of self-interactions, but the geometrical nature of string interactions may enable us to find the full nonlinear invariance of string in this framework. This is our motivation for probing the general properties of reparametrization invariant $2 \mathrm{~d}$ quantum field theory. The most general reparametrization invariant action involving only operators 
of dimension two or less is

$$
\begin{aligned}
S=\frac{1}{4 \pi \alpha^{\prime}} \int \mathrm{d}^{2} z\left[\sqrt{g} g^{a h} \partial_{a} X^{\mu} \partial_{b} X^{\nu} G_{\mu \nu}\right. & (x)+\varepsilon^{a b} \partial_{a} X^{\mu} \partial_{b} X^{\nu} B_{\mu \nu}(x) \\
& \left.\times \alpha^{\prime} \sqrt{g} R^{(2)} \Phi(x)+\sqrt{g} f(x)\right] .
\end{aligned}
$$

$G_{\mu \nu}, B_{\mu \nu}, \Phi$, and $f$ are the background metric, antisymmetric tensor, dilaton and tachyon field expectation values, respectively. We again choose conformal gauge $\sqrt{g} g^{a b}=\sqrt{\hat{g}} \hat{g}^{a b}\left(=\delta^{a b}\right.$ locally $)$. The spacetime metric is still of indefinite signature, so we still need the residual conformal invariance to ensure the decoupling of negative-norm states. In an interacting theory such as (101) this only holds if the stress-energy tensor is traceless. The general form of the trace is

$$
T_{z \bar{z}}=\beta^{f}(x) \sqrt{g}+\beta^{\Phi}(x) \sqrt{g} R^{(2)}+\beta_{\mu \nu}^{G}(x) \partial_{z} X^{\mu} D_{\bar{z}} X^{\nu}+\beta_{\mu \nu}^{\mathrm{B}}(x) \partial_{z} X^{\mu} \partial_{\bar{z}} X^{\nu}
$$

Polyakov [8] consider the special case where a string propagates in a flat space of less than 26 dimensions and showed that the anomaly $\beta^{\Phi}=(26-d) / 48 \pi^{2}=\frac{1}{12} c$ generates an effective dynamics for the scale part of the metric $\mathrm{e}^{\sigma}$. One thus has a conformally invariant theory in which the local scale is coupled. In principle such a dynamics could restore the local scale invariance of the $2 \mathrm{~d}$ quantum field theory even in the general background (101), but in practice no acceptable quantization of the resulting Liouville-type theory has been found in the required range $1<c<25$, despite many attempts [73]. Consequently we shall ask that the scale of the metric completely decouples, leaving a Weyl-invariant two-dimensional theory. (We distinguish Weyl transformations $G_{a b} \rightarrow \Lambda(z, \bar{z}) \cdot g_{a b}$ from conformal changes of coordinate $z \rightarrow f(z)$.) This is desirable in any event since the scale factor acts as a longitudinal coordinate of the string and shifts the ground state energy, so that in general the graviton would be massive, leading to a breakdown of general covariance in the target space $M$. Weyl invariance requires that each of the different coefficient functions $\beta_{\mu \nu}^{G}$ etc., must vanish. Let us ignore for the moment the tachyon coupling $\beta^{f}$. If we then calculate $T_{z \bar{z}}$ as a power series in the loop coupling constant $\alpha^{\prime}$, to leading order we find [27]

$$
\begin{aligned}
& \beta^{\Phi}=\frac{1}{\alpha^{\prime}} \frac{d-26}{48 \pi^{2}}+\frac{1}{16 \pi^{2}}\left[4\left(\nabla^{2} \Phi\right)^{2}-4\left(\nabla^{2} \Phi\right)-R+\frac{1}{12} H^{2}\right]+\mathrm{O}\left(\alpha^{\prime}\right), \\
& \beta_{\mu \nu}^{G}=R_{\mu \nu}-\frac{1}{4} H_{\mu}^{\lambda \sigma} H_{\nu \lambda \sigma}+2 \nabla_{\mu} \nabla_{\nu} \Phi+\mathrm{O}\left(\alpha^{\prime}\right), \\
& \beta_{\mu \nu}^{\mathrm{B}}=\nabla_{\lambda} H_{\mu \nu}^{\lambda}-2\left(\nabla_{\lambda} \Phi\right) H_{\mu \nu}^{\lambda}+\mathrm{O}\left(\alpha^{\prime}\right) .
\end{aligned}
$$

These are precisely the equations of motion of Einstein gravity coupled to an 
antisymmetric tensor gauge field $\left(H_{\mu \nu \lambda}=3 \nabla_{[\mu} B_{\nu \lambda]}\right)$ and dilaton. Higher-loop terms give higher derivative corrections to the equations of motion, scaled by the appropriate power of $\alpha^{\prime}$. A solution to all orders is a conformal field theory. The value of the Schwinger term in the Virasoro algebra is $c=24 \pi^{2} \beta^{\Phi}$; the connection to eq. (9) is provided by the conservation law $\nabla_{\bar{z}} T_{z z}=\nabla_{z} T_{\bar{z} z}$, which when varied with respect to $g$ yields

$$
\begin{aligned}
\nabla_{\bar{z}}\left\langle T_{z z} T_{w w}\right\rangle & =\frac{-\delta}{\delta g_{w w}} \nabla_{z}\left[\beta^{\Phi} \sqrt{g} R+\cdots\right] \\
& =-\beta^{\Phi} \nabla_{z}^{3} \delta(z-w)+\cdots \\
& =6 \beta^{\Phi} \nabla_{\bar{z}} \frac{1}{(z-w)^{4}}+\cdots
\end{aligned}
$$

The other terms in the trace also appear on the r.h.s. of (103), so calculating the closure of the Virasoro algebra is an alternative route to deriving the equations (102). There are solutions to these equations, such as group manifolds with a Wess-Zumino coupling [74,35], for which $\beta^{\Phi}$ receives corrections to all orders. Since $c$ is the more fundamental parameter of string theory, the fact that the dimension of the manifold must differ from 26 for such solutions is not necessarily of concern - all that matters is that $c_{\text {total }}=0$.

Given a solution to (102), we may calculate the traceless part of the stress tensor

$$
T_{z z}=\partial_{z} X^{\mu} \partial_{z} X^{\nu} G_{\mu \nu}(x)-\alpha^{\prime} \partial_{z}^{2} \Phi(x)
$$

and similarly for $T_{\bar{z} \bar{z}}$. These generate the Virasoro algebra, from which we may calculate the spectrum of anomalous dimensions of fields and tensors; to lowest order, the anomalous dimension operator is just the covariant laplacian on the target manifold [75]

$$
h_{\mathrm{F}(x)}=\nabla^{2} F(x)-(\nabla \phi) \cdot \nabla F+\mathrm{o}\left(\alpha^{\prime}\right) .
$$

(Tensor fields gain the appropriate covariantization of this equation.)

The above analysis demonstrates that the imposition of conformal invariance generates the correct equations of motion for the massless fields. We might therefore hope that conformal invariance is the principle governing the dynamics of all the excitations of the string. An elementary example is the tachyon coupling $f(x)$. In order that the scale factor decouples from this term, $f(x)$ must have an anomalous dimension (cf. eq. (104)) $h=(1,1)$ and so we find an equation of motion

$$
\left(-1+\alpha^{\prime} \nabla^{2}\right) f(x)=\mathrm{O}\left(\left(\alpha^{\prime}\right)^{2}\right) .
$$


Thus by arranging for the anomalous contributions to an operator's scaling dimension to compensate the canonical dimension, a coupling which naively cannot occur in a scale-invariant theory is tolerable. One problem with this idea is that, since $f(x)$ is a relevant operator, it cannot contribute to the metric equation of motion in standard renormalization schemes. A similar case can be made for the massive tensor fields of the string, although here the introduction of a single tensor field coupling induces all the others (subject to symmetry considerations) since the massive fields correspond to "nonrenormalizable" terms in the action. Still, each tensor field $T_{\mu \nu \ldots \alpha \beta \ldots} \partial X^{\mu} \partial X^{\nu} \ldots \bar{\partial} X^{\alpha} \bar{\partial} X^{\beta} \ldots$ generates a contribution to the trace of the stress-energy tensor whose leading term $\left(m^{2}+\alpha^{\prime} \nabla^{2}\right) T=\mathrm{O}\left(\left(\alpha^{\prime}\right)^{2}\right)$ is the appropriate equation of motion; in Minkowski space $T_{\mu \ldots \alpha} \ldots$ can have a negative anomalous dimension (e.g. $\mathrm{e}^{i k \cdot X}$ in flat space can have $k^{2}<0$ ) which cancels the positive conformal weight of all the derivatives. In this way it is possible to view conformal invariance as the dynamical principle which yields covariant classical equations of motion for all components of the string background. Again we may in principle compute to all orders the spectrum of highest weight states of the conformal algebra. Those with $h=(1,1)$ will be vertex operators for emitting string fluctuations about the given background. There are two ways to see that conformal invariance implies the equations of motion for the background. First, the $\mathrm{SL}_{2}$ invariance of correlation functions on the sphere guarantees that the background is classically stable against string emission into the vacuum; vertex one-point functions vanish by scale invariance [26]. Second, conformal invariance guarantees the unitarity of the tree-level $S$-matrix; unitarity implies the absence of transitions to the vacuum. Multipoint correlations are given in terms of sums over the operator product coefficients $C_{i j}^{k}$ (cf. eq. (14)). Just as in flat space, the conformal field theory on a sphere defines a conformal field theory on higher genus surfaces. Working on the covering space $\tilde{\tilde{s}}$, the operators in a correlation function may be copied into each fundamental region by the action of the group $\Gamma$. This $\infty$-point correlation is then calculated using a suitable summation prescription as in the free field case. This generalization of the image method gives a route to calculating string perturbation theory in an arbitrary background. Of course, it would be simpler if we had an exact, rather than perturbative, solution to the theory on the sphere. Such a solution might be provided by bootstrap techniques, where the crossing symmetry relation (14) provides a set of nonlinear equations for the exact anomalous dimensions and operator product coefficients. The bootstrap procedure does not in any way lean on perturbation theory in $\alpha^{\prime}$, so strongly coupled sigma models might be analyzed in this manner.

Generically, the string perturbation expansion about a background will be divergent due to vacuum instabilities: tachyon emission, one-loop dilaton tadpoles, etc. [71]. These might be cured if we knew how to shift the vacuum, but because the first-quantized theory is restricted to be on-shell, and the off-shell continuation remains unknown, we don't yet have the technology to proceed along these lines. 
The spacetime supersymmetry of superstrings provides a way out. The argument given in sect. 6 is certainly generalizable to curved backgrounds provided they admit a conserved supersymmetry charge. Thus, until supersymmetry is broken, the background-field fermionic string theory is free of fatal diseases. Therefore let us generalize our discussion to the case of heterotic strings in a curved background (the type II superstring theories are just as straightforward). The structure will be that of a conformal field theory in $\bar{z}$, and a superconformal field theory in $z$. A conformal gauge action for massless backgrounds is $[27,25]$

$$
\begin{aligned}
S=\frac{1}{4 \pi \alpha^{\prime}}\left[\int \mathrm{d}^{2} z \mathrm{~d} \theta(\right. & {\left[G_{\mu \nu}(X)+B_{\mu \nu}(X)\right] D X^{\mu} \partial_{\bar{z}} X^{\nu} } \\
& \left.+\alpha^{\prime} \sqrt{g} R^{(2)} \Phi(X)+A_{\mu}^{a}(X) D X^{\mu} j_{\bar{z}}^{a}\right) \\
& \left.+\int \mathrm{d}^{2} z\left(\Psi_{\mu}^{\alpha}(X) V_{\alpha}^{\mathrm{F}} \partial_{\bar{z}} X^{\mu}+\lambda_{a}^{\alpha}(X) V_{\alpha}^{\mathrm{F}} j_{\bar{z}}^{a}+\alpha^{\prime} \chi^{\alpha}(X) V_{\alpha}^{\mathrm{F}} \omega_{\bar{z}}^{(2)}\right)\right] .
\end{aligned}
$$

Here $G_{\mu \nu}, B_{\mu \nu}$, and $\Phi$ are the bosonic components of the $d=10, N=1$ supergravity multiplet, with $\Psi_{\mu}^{\alpha}$ and $\chi^{\alpha}$ their fermionic partners; and $A_{\mu}^{a}, \lambda^{\alpha}$ from an $N=1$, $\mathrm{E}_{8} \times \mathrm{E}_{8}$ or $\mathrm{SO}(32)$ Yang-Mills multiplet. $j_{z}^{a}$ is the corresponding chiral world sheet affine algebra current, $\omega_{\bar{z}}^{(2)}$ is the world-sheet spin connection, and $V_{\alpha}^{\mathrm{F}}$ represents the fermion vertex, with the spacetime dependence $u^{\alpha}(k) \mathrm{e}^{i k \cdot x}$ replaced by the corresponding background field. A term effecting the quantization of the current algebra $j_{\cong}^{a}$ must also be included. All the terms (apart from the dilaton) are couplings to gauge and super-Poincare currents (modulo the caveats of sect. 6). Note that the action contains only terms with even fermion parity $\Gamma=1$. The traceless (super) stress-energy tensors are

$$
\begin{aligned}
& T_{\bar{z} \bar{z}}=-\frac{1}{2} \partial_{\bar{z}} X^{\mu} \partial_{\bar{z}} X^{\nu} G_{\mu \nu}(X)+\alpha^{\prime} \partial_{\bar{z}} \Phi(X)+\partial_{\bar{z}} X^{\mu} j_{\bar{z}}^{a} A_{\mu a}(X)+j_{\bar{z}}^{a} j_{\bar{z}}^{a}, \\
& T_{z z}=-\frac{1}{2}\left[\partial_{z} X^{\mu} \partial_{z} X^{\nu}+\left(\partial_{z} \psi^{\mu}\right) \psi^{\nu}\right] G_{\mu \nu}(X)+\alpha^{\prime} \partial_{z}^{2} \Phi(X), \\
& T_{z \theta}=-\frac{1}{2} \partial_{z} X^{\mu} \psi^{\nu} G_{\mu \nu}(X)+\alpha^{\prime} \partial_{z}\left[\nabla_{\mu} \Phi(X) \psi^{\mu}\right] .
\end{aligned}
$$

Consider first the case where the fermion couplings $\Psi_{\mu}^{\alpha}, \lambda_{a}^{\alpha}$, and $\chi^{\alpha}$ are absent; the action describes a standard nonlinear sigma model, and again consistency forces the trace of the stress-energy tensor to vanish. A perturbative calculation [27,25] again yields (102) with $H \rightarrow \tilde{H}=H+A \wedge F-\omega \wedge R$, the modification being due to a contribution to the Wess-Zumino coupling $B_{\mu \nu}$ from the sheet fermion chiral anomaly $[76,27,25]$. There is in addition a new counterterm $\delta T_{z \bar{z}}=\beta_{\mu u}^{A} \partial X^{\mu} j_{\bar{z}}^{a}+s s y m$ completion which yields the gauge field equation of motion

$$
\beta_{\mu a}^{A}=\nabla^{\nu} F_{\nu \mu}-2\left(\nabla^{\nu} \Phi\right) F_{\nu \mu}+F_{\mu \lambda} H_{\mu}^{\nu \lambda}+o\left(\alpha^{\prime}\right)
$$


and a contribution $-\frac{1}{2} \alpha^{\prime} F_{\mu}^{\lambda} F_{\lambda v}$ to the Einstein/dilaton equation $\beta^{G}$, and so on. Thus an all orders solution to $\beta^{G}=\beta^{B}=\beta^{\Phi}=\beta^{A}=0$ would again be a conformal field theory appropriate for the heterotic string. Given a sigma model, one must compute the spectrum of anomalous dimensions and the set of operator product coefficients. Highest weight vectors of (super) Virasoro with $h=(1,1)$ are vertex operators.

Now let us consider the inclusion of the fermion backgrounds. These involve the use of spin fields in the action and are thus not written in terms of the more conventional superfields. However, we project onto the spin model $\Gamma=1$ to obtain the fermionic string theory, and since only such terms have been written in (101), the action describes a local field theory. Of course the fermion vertex involves an infinite set of different copies of the same operator in different sea levels. Our rules for manipulating the fermion vertex dictate that in graphs one chooses just one representative configuration out of all the possible combinations of fermion vertices. Although we have not carried out explicit calculations in the presence of spacetime fermion backgrounds, we are confident that no difficulties will arise - one is merely perturbing a local scale invariant field theory by a marginal operator, the fermion vertex. The only novelty is that the spin model does not (yet) have a lagrangian formulation, which makes the analysis somewhat awkward. Because it necessarily includes ghost spin fields, the proper condition for consistency of the sigma model will be

$$
\left\langle Q_{\mathrm{BRST}}^{2}\right\rangle=0
$$

The BRST current contains among other things the traceless stress tensor, so via (103) we again reproduce the bosonic equations (102). The fermion contributions to these equations come from sigma model loop graphs with some (even) number of fermion vertex insertions. The equations of motion for the fermions themselves follow from the fact that $T_{z \theta}$ (eq. (51)) acts on spin fields as the Dirac or Rarita-Schwinger operator. For instance, in order that the "classical" action (101) is BRST invariant to lowest order, the part $Q_{\mathrm{BRST}}^{(1)}=\oint \gamma^{\theta} T_{z \theta}$ of the BRST charge must commute with the lagrangian density. Using (72) and (82) we find schematically

$$
\left[Q_{\mathrm{BRST}}^{(1)}, \lambda_{a}^{\alpha}(x) \mathrm{e}^{-\phi / 2} S_{\alpha} j_{\bar{z}}^{a}\right] \approx \gamma_{\beta \boldsymbol{\alpha}}^{\mu} \nabla_{\mu} \lambda_{a}^{\alpha}(x) \eta \mathrm{e}^{\phi / 2} S^{\beta} j_{\bar{z}}^{a}+\cdots
$$

and similarly for the other fermion backgrounds. If our identification of the Faddeev-Popov ghost $\kappa$ is correct, this is equivalent to demanding invariance under the local fermionic symmetries of the GSS action.

The sigma model thus gives an elegant covariant approach to the study of solutions of the string equations. Of particular interest are solutions with the topology $\mathbf{M}_{4} \times 9$ for some compact six-manifold 9 , which could be the starting point for phenomenology. Ricci-flat Kähler six-manifolds of SU(3) holonomy (which 
have been dubbed Calabi-Yau spaces $[77,26]$ ) are believed to yield conformally invariant sigma models [78] with $N=2$ world-sheet supersymmetry. These spaces have a rather beautiful geometrical structure which profoundly influences physics below the compactification scale. We would like to see this structure emerge from the sigma model, and indeed it does. Massless particles in Kaluza-Klein field theory correspond to zero-modes of the kinetic operator on 9 . In the Ramond sector of the corresponding sigma model, the string generalization of the kinetic operator is the Dirac-Ramond operator $G_{0}=\int P \cdot \psi$ which also generates global supersymmetry on the cylinder. The supersymmetry index $\operatorname{tr}(\Gamma)$ thus counts the difference in the number of left- versus right-handed massless fermion states (i.e. conformal spin fields with $G_{0}=h-\frac{1}{16} \hat{c}=0$ ). In his original paper on the index, Witten [51] demonstrated that

$$
\operatorname{tr}(\Gamma)=\chi(\mathscr{B}),
$$

i.e. the index is just the Euler number of 9 . In the Calabi-Yau case, anomaly cancellation requires embedding the spin connection in the gauge group, which breaks $\mathrm{E}_{8} \times \mathrm{E}_{8}^{\prime} \rightarrow \mathrm{E}_{6} \times \mathrm{E}_{8}^{\prime}$. The gauge fermions of $\mathrm{E}_{8}$ then transform as $248 \rightarrow$ $(8,1)+(3,27)+(\overline{3}, \overline{27})+(2,78)$ under $\mathrm{E}_{8} \times \mathrm{SU}(3)$, and the index tells us there are therefore at least $\chi(\mathscr{B})$ families of 27 's of $\mathrm{E}_{6}$ (or $\overline{27}$ 's, depending on the sign of $\chi$ ).

The complex Kähler structure of Calabi-Yau spaces has been used to expand the information available from index theorems. The de Rham complex (i.e. the collection of operators relevant to the Dirac index) splits up into pieces classified by the U(1) charge defined by the complex structure. This refinement of cohomology (Dolbeault cohomology) allows one to count the number of families and antifamilies separately [26]. Correspondingly, whenever the sigma model manifold admits a global symmetry such as the $\mathrm{U}(1)$ of the complex structure, one may compute a refinement of the supersymmetry index - the character valued index $\operatorname{tr}\left(\Gamma \mathrm{e}^{i \pi H}\right)[79]$. The relevant $\mathrm{U}(1)$ charge is the global component of the current $\left(J_{m n}(Y)\right.$ is the complex structure on 9 )

$$
h=J_{m n}(Y) \psi^{m} \psi^{n}, \quad H=\oint h,
$$

which is the lowest component of the $N=2$ stress tensor multiplet (cf. eq. (66)). The character valued index allows us to count separately the zero modes in each charge sector, which is exactly the Dolbeault refinement of de Rham cohomology.

The sigma model index theorems are valid in the Ramond sector; in the NS sector there are no zero modes on which to base the index theorem and so the index typically does not apply. On the other hand, in special cases such as Calabi-Yau there may be $h=(1,0)$ spin fields whose line integral is a conserved supersymmetry charge. The commutator of this charge with the massless fermion vertices will then generate vertices for massless bosons. 
Vertex operators in the sigma model factorize into the form $V^{(4)}\left(X^{\mu}\right) \cdot V^{(6)}\left(Y^{m}\right)$ where $X^{\mu}$ and $Y^{m}$ parametrize $\mathrm{M}_{4}$ and 9 respectively; vertices for massless particles come from conformal fields on 9 with $h, \bar{h}=0$ or 1 . For example, the dilaton which controls the radius of $\mathscr{B}$ corresponds to the vertex $\left(G_{m n}\right.$ is the metric on 6 )

$$
V_{\mathrm{dil}, \mathscr{G}}=\mathrm{e}^{i k \cdot X} G_{m n}(Y) \partial_{z} Y^{m} \partial_{\bar{z}} Y^{n}+\text { ssym completion. }
$$

Each zero-mode of the Lichnerowicz operator on 9 gives rise to a massless four dimensional gravitational scalar in this way. Kähler sigma models also have instantons; the Kähler form is a source of antisymmetric tensors $B_{m n}$ which are closed but not exact, and the $B_{m n}$ coupling in the action is precisely the instanton density. The vertex

$$
V_{\text {axion }}=\mathrm{e}^{i k \cdot X_{B_{m n}}}(Y) \partial_{z} Y^{m} \partial_{\bar{z}} Y^{n}
$$

thus emits four-dimensional axions since $B_{\mu \nu}$ couples to $F \tilde{F}$ through the ChernSimons term (with gravitational strength because the fields originate in the tendimensional gravitational multiplet). In general, the whole range of massless fields is determined by index theorems for suitable families of operators on $\Re$. The fermionindices decompose under $\mathrm{SU}(3)$ as $16 \rightarrow 3+3+\overline{3}+\overline{3}+4$ singlets. The singlets form a four-dimensional massless spinor for each of the families, whose vertex operator is

$$
V_{\text {matter }}=\mathrm{e}^{i k \cdot X} \lambda_{a}^{\alpha}(Y, k) V_{\alpha}^{F} j_{\bar{z}}^{a}
$$

with the $a, \alpha$ indices reflecting the above considerations. Similar analysis yields the remaining four-dimension gravitino vertex and the unbroken SU(3) singlet supersymmetry charges $\boldsymbol{Q}_{\alpha}$. The vertices for the scalar partners of these matter fermions are then obtained from the operator product of the supersymmetry current with the fermion vertices. Supersymmetry (or for that matter any Killing vector of the compact space) is a nongeneric property of the nonlinear model. It requires the existence of an $h=(1,0)$ spin field (a current). On the other hand, massless particles are somewhat more generic, requiring only the existence of $h=(1,1)$ operators.

Recently it has been discovered in a field theoretic analysis that the Yukawa couplings of the massless fields are also topologically determined as intersection matrices of differential forms [79a]. If this is indeed a property of the full string theory and not just an approximation, then conformal field theory has an even deeper structure to it. Yukawa couplings of the low-energy field theory are the overlaps of products of three string wave functions - they are precisely the operator product coefficients of the massless matter vertices. It is a rather remarkable and perhaps deep property of two-dimensional field theory that it encodes so much information about geometry in its algebraic structure. Perhaps enough information can be obtained from topology to determine a solution (or a family of solutions) to the sigma model boostrap. Then the entire tree-level structure of the effective theory 
at the compactification scale would be known - masses, mixings, coupling constants, etc.! Certain special cases already lend themselves to analysis. In a certain (orbifold) limit [80], some Calabi-Yau space sigma models are exactly soluble. The spacetime manifold is essentially a "twisted" torus, i.e. the quotient of a torus by a group of transformations (twists) which has fixed points. At the fixed point the periodic identification under the group generates conical-like singularities, loosely speaking. In addition to the states created by the standard conformal fields on the torus, there are states which are pinned to the fixed points of the twisting operation. These states are created by conformal fields that are called twist operators. Like the spin operator of the fermionic string, these operators have a nonlocal operator product with the string coordinates parametrizing the torus, thus changing their mode expansion. The operator product coefficients of the twist operators for these spaces can be determined using the conformal algebra and differential equation techniques [81]. The rich structure of the conformal algebra (and in particular its $N=2$ supersymmetric extension) is just beginning to be tapped in the search for string compactifications.

Finally, we mention in passing that there is one other known class of conformally invariant sigma models on which the fermionic string may be compactified - group manifolds with a Wess-Zumino interaction [82]. The resulting supersymmetric current algebra may be analyzed using the conformal and group structures; unfortunately one finds [83] that there are no $h=\frac{1}{16} \hat{c}$ spin fields, i.e., supersymmetry is broken in the Ramond sector. This is not too surprising because group manifolds have zero Euler number, so the index $\operatorname{tr}(\Gamma)$ vanishes. Thus all fermions acquire a mass of order the compactification scale, which is phenomenologically unacceptable.

\section{Conclusions}

Conformal field theory provides an impressive tool with which to probe the structure of string theory. We have reworked and completed the covariance quantization of fermionic string theories, relying heavily on conformal methods. These methods express the dynamics of string in terms of the local properties of scale invariant $2 \mathrm{~d}$ quantum field theory. The fermion vertex operator, a rather opaque object in previous formulations, acquires a certain simplicity when its current algebraic properties are exploited. The full (on-shell) supersymmetry of the theory has become more manifest and manageable, with the supersymmetry charge expressed as the line integral of a dimension $(1,0)$ conformal field on the string world sheet. Deformations of the contour along which this and other currents are integrated reveals a number of interesting results: vanishing of massless particle tadpoles and mass renormalizations, decoupling of spurious states from scattering amplitudes, and the equivalence of the various vertices in different ghost Bose seas. String-loop perturbation theory is relatively straightforward, requiring only minor modifications of previous formulations. Barring some unforeseen catastrophe, finiteness is a rather simple consequence of modular invariance the nonrenormalization theorems just mentioned. 
One of the attractive features of the conformal approach is its universal applicability. Eventually we will need to understand the properties of string in relation to spacetime geometry and gauge symmetries. In the presence of nontrivial background field configurations, one cannot use arguments which rely on properties peculiar to flat space string theory, such as simple mode expansions, decoupling of the $z$ and $\bar{z}$ dependence, and so on. That is why we have tried to present the analysis in terms of generic properties - the contour deformation arguments, for instance, require only that there exist a dimension $(1,0)$ field whose line integral is conserved and can be applied to any symmetry which gives rise to such a field. As a conceptual framework, conformal field theory provides an arena for string dynamics which needs to be explored; it has already yielded some results in particularly simple cases (group manifolds and orbifolds). We believe the work presented here has laid a solid foundation for these further investigations.

We are grateful to T. Banks, C. Callan, J. Cohn, V. Dotsenko, P. Goddard, D. Olive, M. Perry, Z. Qiu, R. Rohm, W. Siegel, and E. Witten for helpful discussions.

\section{References}

[1] H. Georgi and S. Glashow, Phys. Rev. Lett. B32 (1974) 438

[2] D.V. Volkov and V.P. Akulov, Phys. Lett. 46B (1973) 109; J. Wess and B. Zumino, Nucl. Phys. B70 (1974) 139

[3] Th. Kaluza, Sitzungsber. Preuss. Akad. Wiss. Berlin K1 (1921) 966; O. Klein, Z. Phys. 37 (1926) 895

[4] V. Allesandrini, D. Amati, M. Le Bellac and D. Olive, Phys. Reports 1C (1971) 170;

S. Mandelstam, Phys. Reports 13C (1974) 259;

J. Scherk, Rev. Mod. Phys. 47 (1975) 123;

J. Schwarz, Phys. Reports 89 (1982) 223

[5] V. Kac, Math. USSR Izv. 2 (1968) 1271;

I. L. Kantor, Sov. Math Dokl. 9 (1968) 409;

J. Moody, J. Algebra 10 (1968) 211

[6] M. Virasoro, Phys. Rev. D1 (1970) 2933;

I.M. Gelfand and D.B. Fuchs, Functs. Anal. Prilozhen 2 (1968) 92;

J. Weis, unpublished (cf. S. Fubini and G. Veneziano, Ann. of Phys. 63 (1971) 12)

[7] S. Fubini and G. Veneziano, Nuovo Cim. 67A (1970) 29;

Y. Nambu. Proc. Int. Conf. on Symmetries and quark models, Wayne State Univ., 1969

[8] A.M. Polyakov, Phys. Lett. 103B (1981) 207, 211

[9] P. Goddard, J. Goldstone, C. Rebbi and C. Thorn, Nucl. Phys. B56 (1973) 109

[10] F. Gliozzi, D. Olive and J. Scherk, Nucl. Phys. B122 (1977) 253

[11] M. Green and J. Schwarz Phys. Lett. 149B (1984) 117

[12] D. Gross, J. Harvey, E. Martinec and R. Rohm, Nucl. Phys. B256 (1985) 253; Princeton preprint (July, 1985)

[13] A.A. Belavin, A.M. Polyakov and A.B. Zamolodchikov, Nucl. Phys. B241 (1984) 333

[14] D. Friedan, Z. Qiu and S. Shenker, Phys. Rev. Lett. 52 (1984) 1575; in Vertex operators in mathematics and physics, ed. J. Lepowsky et al. (Springer, 1984); Phys. Lett. 151B (1985) 37

[15] D. Friedan, E. Martinec and S. Shenker, Phys. Lett. 160B (1985) 55

[15a] V. Knizhnik, Phys. Lett. 160B (1985) 403

[16] M. Kato and K. Ogawa, Nucl. Phys. B212 (1983) 443;

S. Hwang, Phys. Rev. D28 (1983) 2614 
[17] K. Fujikawa, Phys. Rev. D25 (1982) 2584

[18] C. Becchi, A. Rouet and R. Stora, Phys. Lett. 52B (1975) 344;

I.V. Tyutin, Lebedev preprint FIAN no. 39 (1975), in Russian, unpublished

[19] P. Ramond, Phys. Rev. D3 (1971) 2415

[20] A. Neveu and J. Schwarz, Nucl. Phys. B31 (1971) 86; Phys. Rev. D4 (1971) 1109

[21] K. Bardacki and M. Halpern, Phys. Rev. D3 (1971) 2493

[22] C. Thorn, Phys. Rev. D4 (1971) 1112;

[22a] J. Schwarz, Phys. Lett. 37B (1971) 315;

E. Corrigan and D. Olive, Nuovo Cim. 11A (1971) 749;

E. Corrigan and P. Goddard, Nuovo Cim. 18A (1973) 339

[23] M. Green and J. Schwarz, Nucl. Phys. B243 (1984) 285

[24] W. Siegel, Nucl. Phys. B263 (1986) 93

[25] C. Lovelace, Phys. Lett. 135B (1984) 75;

D. Friedan and S. Shenker, unpublished talk at the Aspen Summer Institute (1984);

D. Friedan, Z. Qiu and S. Shenker, Proc. APS Div, of Particles and fields Conf., Santa Fe (1984),

ed. T. Goldman and M. Nieto (World Scientific, 1984);

E. Fradkin and A. Tseytlin, Phys. Lett. 158B (1985) 316;

A. Sen, Fermilab preprints (1985)

[26] P. Candelas, G. Horowitz, A. Strominger and E. Witten, Nucl. Phys. B258 (1985) 46

[27] C. Callan, D. Friedan, E. Martinec and M. Perry, Princeton preprint (June 1985) to be published in Nucl. Phys. B

[28] T. Banks and M. Peskin, Proc. Argonne Symposium on Anomalies, geometry, and topology, ed. W. Bardeen and A. White (World Press, 1985); and SLAC-PUB-3740;

M. Kaku and J. Lykken, Proc. Argonne Symposium

D. Friedan. Chicago preprint EFI 85/27;

W. Siegel and B. Zwiebach, Nucl. Phys. B263 (1986) 105;

K. Itoh, T. Kugo, H. Kumimoto and H. Ooguri, Kyoto preprint KUNS-800-HE(TH)85/04;

D. Friedan. Phys. Lett. 162B (1985) 102

[29] L. Brink, P. Di Vecchia and P. Howe, Phys. Lett. 65B (1976) 471;

S. Deser and B. Zumino, Phys. Lett. 65B (1976) 369

[30] E. Martinec, Phys. Rev. D30 (1984) 1198

[31] R. Brower, Phys. Rev. D6 (1972) 1655

[32] P. Goddard and C. Thorn, Phys. Lett. 40B (1972) 235

[33] E. Fradkin and T. Tseytlin, Phys. Lett. 106B (1981) 63

[34] P. Goddard and D. Olive, in Vertex operators in mathematics and physics, MSRI publication no. 3, ed. J. Lepowsky et al. (Springer, 1984)

[35] V. Knizhnik and A.B. Zamolodchikov, Nucl. Phys. B247 (1984) 83

[36] D. Olive and N. Turok, DAMTP preprint (1984)

[36a] J.-L. Gervais and B. Sakita, Nucl. Phys. B34 (1971) 477

[37] L. Brink, D. Olive and J. Scherk, Nucl. Phys. B61 (1973) 173

[38] D. Friedan, in 1982 Les Houches summer school, Recent advances in field theory and statistical mechanics, ed. Z. Zuber and R. Stora (North-Holland, 1984)

[39] D.B. Ray and I.M. Singer, Adv. Math. 7 (1971) 145; Ann. Math. 98 (1973) 154;

L. Brown and J. Cassidy, Phys. Rev. D16 (1977) 1712

[40] S. Coleman, Comm. Math. Phys. 31 (1973) 259

[41] Z. Koba and H.B. Nielsen, Nucl. Phys. B12 (1969) 517

[42] O. Alvarez, Nucl. Phys. B216 (1983) 125

[43] I. Batalin and G. Vilkovisky, Phys. Lett. 69B (1977) 309;

E. Fradkin and G. Vilkovisky, Phys. Lett. 73B (1978) 209

[44] S. Fubini, D. Gordon and G. Veneziano, Phys. Lett. 29B (1969) 679;

L. Susskind, Phys. Rev. D1 (1970) 1182

[45] C. Lovelace, Phys. Lett. 32B (1970) 703;

V. Alessandrini, Nuovo Cim. 2A (1971) 321;

V. Alessandrini and D. Amati, Nuovo Cim. 4A (1971) 793

[46] B. Riemann, Theorie der Abel'schen Funktionen, Gesselte Mathematische Werke und Wissenschaftlichen, 2nd ed. (Dover, NY, 1953) pp. 88-142 
[47] D. Gross, A. Neveu, J. Scherk and J. Schwarz, Phys. Rev. D2 (1970) 697

[48] G. Moore and P. Nelson, Nucl. Phys. B266 (1986) 58;

E. d'Hoker and D.H. Phong, Columbia preprint CU-TP-323

[49] D. Fairlie and D. Martin, Nuovo Cim. 21A (1974) 647;

L. Brink and J.O. Winnberg, Nucl. Phys. B103 (1976) 445

[50] M. Bershadsky, V. Knizhnik and M. Teitelman, Phys. Lett. 151B (1985) 31

[51] E. Witten, Nucl. Phys. B202 (1982) 253

[52] E. Witten, in Proc. Argonne Symposium on Anomalies, geometry and topology

[52a] S. Mandelstam, Phys. Lett. 46B (1973) 447

[53] J. Cohn, D. Friedan, Z. Qiu and S. Shenker, to appear

[54] H. Sugawara, Phys. Rev. 170 (1968) 1659

[55] R. Dashen and Y. Frishman, Phys. Rev. D11 (1975) 2781

[56] L. Brink, D. Olive, C. Rebbi and J. Scherk, Phys. Lett. 45B (1973) 379; D. Olive and J. Scherk, Nucl. Phys. B64 (1973) 334;

J. Schwarz and C.C. Wu, Phys. Lett. 47B (1973) 453;

E. Corrigan, P. Goddard, D. Olive and R. Smith, Nucl. Phys. B67 (1973) 477

[57] M. Halpern, Phys. Rev. D12 (1975) 1684;

T. Banks, D. Horn and H. Neuberger, Nucl. Phys. B108 (1976) 119;

I. Frenkel and V. Kac, Invent. Math. 62 (1980) 23:

(j. Segal, Comm. Math. Phys. 80 (1981) 301;

P. Goddard and D. Olive, in Vertex operators in mathematics and physics, ed. J. Lepowsky et al. (Springer, 1984)

[58] Ref. [17] of Siegel and Zweibach (ref. [28])

[59] S. Mandelstam, Nucl. Phys. B69 (1974) 77

[60] E. Martinec, Phys. Rev. D28 (1983) 2604

[61] A. Chodos and C. Thorn, Nucl. Phys. B72 (1974) 509;

B.L. Feigin and D.B. Fuchs, Moscow preprint (1983); Funct. Anal. Appl 16 (1982) 114;

V.L. Dotsenko and V. Fateev, Nucl. Phys. B240 (1984) 312;

C. Thorn, Nucl. Phys. B248 (1985) 551

[62] A. Neveu, J. Schwarz and C. Thorn, Phys. Lett. 35B (1971) 529

[63] M. Green and J. Schwarz, Phys. Lett. 109B (1982) 444

[64] E. Witten, Proc. 4th Workshop on Grand unification, Philadelphia, ed. H.A. Weldon et al. (Birkhausen, 1983);

R. Shankar, Phys. Lett. 92B (1980) 333

[65] T. Banks, D. Friedan, E. Martinec, M. Peskin and C. Preitschopf, SLAC-PUB-3853

[66] K. Kikkawa, B. Sakita and M. Virasoro, Phys. Rev. 184 (1969) 1701

[67] E. Martinec, in preparation

[68] L. Bers, Bull. Am. Math Soc. 5 (1981) 131

[69] L. Ford, Automorphic Functions (Chelsea, 1951)

[70] M. Green and J. Schwarz, Phys. Lett. 109B (1982) 444

[71] J. Shapiro, Phys. Rev. D11 (1975) 2937; M. Ademollo et al., Nucl. Phys. B94 (1975) 221

[72] E.C.G. Stuckelberg, Helv. Phys. Acta 15 (1942) 33;

R.P. Feynman, Phys. Rev. 80 (1950) 440;

J. Schwinger, Phys. Rev. 82 (1951) 664

[73] J-L. Gervais and A. Neveu, Nucl. Phys. B199 (1982) 50, B209 (1982) 125, B224 (1983) 320, B238 (1984) 125, B238 (1984) 301;

R. Marnelius, Nucl. Phys. B211 (1983) 14, B221 (1983) 409;

E. D'Hoker and J. Jackiw, Phys. Rev. D26 (1982) 3517;

E. D'Hoker, D.Z. Freedman and R. Jackiw, Phys. Rev. D28 (1983) 2583;

T. Curtright and C. Thorn, Phys. Rev. Lett. 48 (1982) 1309;

E. Braaten, T. Curtright and C. Thorn, Phys. Lett. B118 (1982) 115;

T. Yoneya, Phys. Lett. 148B (1984) 111

[74] E. Witten, Comm. Math. Phys. 92 (1984) 451 
[75] D. Friedan, Berkeley PhD thesis: Ann. of Phys., to be published

[76] C. Hull and E. Witten, Princeton preprint (June 1985)

[77] E. Calabi, in Algebraic geometry and topology: a Symposium in honor of S. Lefschetz (Princeton Univ. Press, 1957) p. 78;

S.-T. Yan, Proc. Natl. Acad. Sci. 74 (1977) 1798

[78] L. Alvarez-Gaumé, S. Coleman and P. Ginsparg, HUTP 85/A037;

L. Alvarez-Gaumé and P. Ginsparg, HUTP 85/A030;

C. Hull, IAS preprints (1985)

[79] E. Witten, J. Diff. Geom. 17 (1982) 661

[79a] A. Strominger and E. Witten, Comm. Math. Phys. 101 (1985) p. 341;

A. Strominger, Santa Barbara preprints (1985)

[80] L. Dixon, J. Harvey, C. Vafa and E. Witten, Nucl. Phys. B261 (1985) 678

[81] Various combinations of L. Dixon, D. Friedan, J. Harvey, E. Martinec and S. Shenker, work in progress

[82] P. di Vecchia, Landau-NORDITA seminar, Moscow (1984), unpublished;

D. Friedan and S. Shenker, talk at the APS Div. of Particles and fields Conf., Santa Fe (1984), ed.

T. Goldman and M. Nieto (World Scientific, 1984);

P. di Vecchia, V. Knizhnik, J-L. Petersen and P. Rossi, Nucl. Phys. B253 (1985) 701;

R. Rohm, Princeton preprint (1984);

T. Curtright and C. Zachos, Phys. Rev. Lett. 53 (1984) 1799

[83] D. Friedan and S. Shenker, unpublished 